Submitted to ApJ 31 Aug 2012; AcCepted 22 Mar 2013

Preprint typeset using $\mathrm{IATEX}_{\mathrm{E}}$ style emulateapj v. 12/16/11

\title{
THE MULTIPHASE STRUCTURE AND POWER SOURCES OF GALACTIC WINDS IN MAJOR MERGERS
}

\author{
DAVID S. N. Rupke ${ }^{1}$ \\ Department of Physics, Rhodes College, Memphis, TN 38112; \\ Institute for Astronomy, University of Hawaii, Honolulu, HI 96822 \\ AND Sylvain Veilleux \\ Department of Astronomy, University of Maryland, College Park, MD 20742 \\ Submitted to ApJ 31 Aug 2012; Accepted 22 Mar 2013
}

\begin{abstract}
Massive, galaxy-scale outflows are known to be ubiquitous in major mergers of disk galaxies in the local universe. In this paper, we explore the multiphase structure and power sources of galactic winds in six ultraluminous infrared galaxies (ULIRGs) at $z<0.06$ using deep integral field spectroscopy with the Gemini Multi-Object Spectrograph (GMOS) on Gemini North. We probe the neutral, ionized, and dusty gas phases using $\mathrm{Na}$ I D, strong emission lines ([O I], H $\alpha$, and [N II]), and continuum colors, respectively. We separate outflow motions from those due to rotation and tidal perturbations, and find that all of the galaxies in our sample host high-velocity flows on kiloparsec scales. The properties of these outflows are consistent with multiphase (ionized, neutral, and dusty) collimated bipolar winds emerging along the minor axis of the nuclear disk to scales of $1-2 \mathrm{kpc}$. In two cases, these collimated winds take the form of bipolar superbubbles, identified by clear kinematic signatures. Less collimated (but still high-velocity) flows are also present on scales up to $5 \mathrm{kpc}$ in most systems. The three galaxies in our sample with obscured QSOs host higher velocity outflows than those in the three galaxies with no evidence for an AGN. The peak outflow velocity in each of the QSOs is in the range $1450-3350 \mathrm{~km} \mathrm{~s}^{-1}$, and the highest velocities $\left(2000-3000 \mathrm{~km} \mathrm{~s}^{-1}\right)$ are seen only in ionized gas. The outflow energy and momentum in the QSOs are difficult to produce from a starburst alone, but are consistent with the QSO contributing significantly to the driving of the flow. Finally, when all gas phases are accounted for, the outflows are massive enough to provide negative feedback to star formation.

Keywords: galaxies: evolution — galaxies: interactions — galaxies: ISM — galaxies: kinematics and dynamics - ISM: jets and outflows - galaxies: quasars
\end{abstract}

\section{INTRODUCTION}

Massive, high-velocity outflows are present in almost all major galaxy mergers in the local universe (Heckman et al. 1990, 2000, Rupke et al. 2002, 2005a b c, Martin 2005, 2006, Westmoquette et al. 2012; Soto \& Martin 2012 , Soto et al. 2012). These kpc-scale outflows exist in both the neutral and ionized gas phases. They have high velocities (typically a few hundred $\mathrm{km} \mathrm{s}^{-1}$, though reaching $>1000 \mathrm{~km} \mathrm{~s}^{-1}$ in some cases); large mass outflow rates (estimates suggest a few tens of percent of the star formation rate); and large extents ( $\gtrsim 5 \mathrm{kpc})$. They have been thought to be powered primarily by star formation (Rupke et al. 2005a) Krug et al. 2010). Their properties are consistent with requirements for negative feedback on star formation and black hole accretion in mergers, as suggested by numerical simulations (e.g., Springel et al. 2005. Hopkins et al. 2005).

However, these conclusions rest on studies using longslit optical spectroscopy of a single gas phase. Such studies do not probe the detailed structures of winds, and have not compared in detail the properties of different gas phases. In particular, disentangling motions in the ionized gas (as probed by strong emission lines) due to rotation, tidal motions, and outflows is difficult without spatially-resolved data. The present ubiquity of sensitive

1'drupke@gmail.com optical integral field spectrographs on large telescopes has made it possible to study the winds in these systems in much greater detail.

Late-stage, major mergers of massive disk galaxies in the local universe are synonymous with ultraluminous infrared galaxies (ULIRGs; e.g., Veilleux et al. 2002). ULIRGs have been studied for many years using integral field spectroscopy (IFS; e.g., Colina et al. 2005, and references therein). However, these early studies used $4 \mathrm{~m}$ class telescopes at relatively low spectral resolution, and did not typically address the outflows in these systems (though see Colina et al. 1999).

Recent deep observations on $8-10 \mathrm{~m}$ class telescopes at high spatial resolution have made possible the detailed study of the multiphase wind structure and power sources in ULIRGs (Arribas et al. 2008; Shih \& Rupke 2010; Rupke \& Veilleux 2011, Westmoquette et al. 2012). Shih \& Rupke (2010) confirmed the outflow velocities, mass, and radial extent measured previously in F10565+2448 (Rupke et al. 2005c; Martin 2006), but also showed that the outflowing gas has a complicated structure that is inconsistent with lifting of rotating gas from the disk (Martin 2006). Rupke \& Veilleux (2011) confirmed the detection of an extended outflow in Mrk 231 (Rupke et al. 2005a) and showed that this outflow emerges in all projected directions and is likely powered by the central QSO. This galaxy may host the 
first known example of QSO-mode feedback in a major merger. (See also Fischer et al. 2010a; Feruglio et al. 2010 for molecular gas detections of the outflow.) Westmoquette et al. (2012) presented the first large-scale IFS survey of outflows in ULIRGs. These authors focused on the ionized gas phase, and detected ubiquitous kiloparsecscale outflows through the presence of broad, blueshifted velocity components. The high velocities they detected in some systems (reaching several thousand $\mathrm{km} \mathrm{s}^{-1}$ ) implicate an AGN in powering the outflows.

In this paper, we present an IFS study of six ULIRGs. This study differs from early ULIRG studies in its high depth, spatial resolution, and spectral resolution. It also differs from the recent study of Westmoquette et al. (2012) by our ability to probe multiple gas phases (neutral and ionized) at higher spatial resolution (enabled by 0 .'2 spatial sampling) and by our modeling of the resulting wind structure. The data on two of these systems were first presented in Shih \& Rupke (2010) and Rupke \& Veilleux (2011), and we re-reduce and reanalyze these data along with four new systems. Five of these systems were first surveyed for outflows using longslit spectroscopy (Heckman et al. 2000; Rupke et al. 2002, 2005a c; Martin 2005).

In Section 2, we discuss our observations and data analysis. We discuss our analysis procedures and wind modeling in Section3 We then detail the properties of individual objects in Section 4 . The results on individual systems are synthesized to form a sample-wide picture in Section 5, with the crucial results found in Figures 49- 56. We summarize in Section6.

\section{SAMPLE, OBSERVATIONS, AND REDUCTION}

The six systems in our sample (Table 1) were selected for their proximity and high infrared luminosity: $z<0.06$ and $\log \left(L_{\mathrm{IR}} / L_{\odot}\right) \gtrsim 12$. All six are members of the Revised Bright Galaxy Sample (RBGS; Sanders et al. 2003), which was assembled based on Infrared Astronomical Satellite data. They span a range of nuclear properties and merger classes, though all are thought to be in the late stages of a major merger (mass ratio $\lesssim 3$; Veilleux et al. 2002).

The galaxies in our study were observed with the Integral Field Unit in the Gemini Multi-Object Spectrograph (GMOS; Allington-Smith et al. 2002, Hook et al. 2004) on the Gemini North telescope. In each case we used the integral field unit in 2-slit mode, with the B600 grating and $r^{\prime}$ filter, yielding wavelength coverage from 5600 to $7000 \AA$ and a spectral resolution averaging $1.6 \AA$ at the central wavelength of $6300 \AA$. (This spectral resolution is measured directly from night sky emission lines in each spaxel.)

To assess image quality and relative photometry in galaxies with an identifiable nucleus, we fit a broad Gaussian to the nucleus in each exposure. Variations in image quality and cloud cover between exposures led to at most a 30\% difference in this peak flux between the highest and lowest fluxes. A small number of exposures with larger variations were discarded. Delivered image quality through the IFS was difficult to assess for most sources, since there are no point sources in the field of view of the integral field unit (except for the QSO in Mrk 231, which has a full width at half maximum, or FWHM, of $0.5-0.6$ ). However, fits to multiple field stars in 12 of the 13 acquisition images in our dataset yield a mean FWHM of $0{ }^{\prime \prime} 59$, with a standard deviation of 0.16 and a range of $0 . ! 4-0$. . 9 .

The native FOV of GMOS in 2-slit mode is $5^{\prime \prime} \times 7^{\prime \prime}$. All sources were observed in two pointings. In four systems, GMOS was dithered along the short axis, with some overlap between the two pointings. In the other two (F08572+3915 and VV 705), it was dithered between two nuclei.

Table 2 lists further details of the observations of each source.

We reduced the data using IRAF v1.14 and v1.10 of the data reduction package provided by the Gemini Observatory. We modified the Gemini software slightly to accommodate error propagation, and supplemented it with custom IDL routines. These IDL routines perform the following steps: (1) correcting single-wavelength images to a common center (due to differential atmospheric refraction); (2) applying a small shift to the wavelength calibration using the line centers of atmospheric emission lines ([O I] 6300, $6364 \AA$ or Na I D 5890, $5896 \AA$ ); (3) telluric correction (using a scaled version of the high SNR Mrk 231 nuclear spectrum as a template); (4) spatial rebinning of spaxels; and (5) mosaicing of dithered positions.

The native 0 ". 2-diameter hexagonal spaxels of GMOS are resampled to 0 .' 1 square spaxels by the GMOS IRAF pipeline. To Nyquist sample the seeing disk while maximizing sensitivity, we binned the 0.11 square pixels into $0^{\prime \prime} .3$ square pixels.

The relative flux calibration of our data is robust. However, flux calibration uncertainties at the red filter transmission edge may affect the $[\mathrm{N} \mathrm{II}] / \mathrm{H} \alpha$ ratio at the $\lesssim 50 \%$ level in F08572+3915, which has the highest redshift in our sample and thus an [N II] line near the filter edge.

The final result is eight data cubes (one per system dithered along the GMOS short axis, and two per system dithered between nuclei). The resulting cubes contain hundreds of spectra.

\section{ANALYSIS AND MODELING}

\subsection{Identification of Nuclei}

The nuclei in these systems are heavily obscured, and the optical continuum peak is often not coincident with the underlying stellar mass peak. The stellar mass peak can be approximated by the location of the near-infrared (NIR) luminosity peak (e.g., Scoville et al. 2000).

Archival HST F814W and F160W images exist for each galaxy in our sample. To identify the nuclei within the GMOS data cubes, we located the NIR peak or peaks in each HST F160W image. We registered the NIR image with the $\mathrm{F} 814 \mathrm{~W}$ image by matching the positions of $1-3$ relatively unobscured nuclear star clusters, as in Iwasawa et al. (2011b). We then convolved each F814W image with a 5-pixel Gaussian to match the average seeing in our data $\left(0^{\prime \prime} 6\right)$. Finally, we matched the convolved F814W and GMOS continuum peaks. The GMOS continuum is slightly bluer than the $\mathrm{F} 814 \mathrm{~W}$ continuum $(R$ vs. $I$ band), but we do not expect the continuum peak to shift significantly over this wavelength range. The resulting nuclear locations in the GMOS data are accurate to $\lesssim 0^{\prime \prime}$. 1 , or a third of a spaxel. 
The exception to this procedure is Mrk 231; the nucleus for this galaxy is easily identifiable as the bright QSO.

\subsection{Continuum, Emission Line, and Absorption Line Fitting}

We modeled the continuum and emission lines in each spectrum using UHSPECFIT, a suite of IDL software which optimizes continuum and emission line fits to spectra. UHSPECFIT is described elsewhere (Rupke et al. 2010). In brief, it masks emission line regions, fits the continuum, and then simultaneously fits all emission lines in the continuum-subtracted spectrum. The fit is improved by repeating this process based on the parameters derived from the first fit.

We fit simple polynomial continua (of order $3-4$ ) to each spectrum. For Mrk 231, we also fit a scaled version of the extremely bright nuclear QSO spectrum (Rupke \& Veilleux 2011). The QSO point spread function (PSF) bleeds into many spaxels and must be removed.

The continua in our galaxies are dominated by young stars, which have weak absorption lines in the wavelength range of our data ( $>5200 \AA$ rest frame). The stellar absorption line that would most affect our emission line fits is $\mathrm{H} \alpha$; because our spectra do not cover other Balmer lines, we cannot constrain the strength of $\mathrm{H} \alpha$ absorption using other lines. However, observations of ULIRGs show that the equivalent width of $\mathrm{H} \beta$ absorption is typically in the range $3-7 \AA$ within $2 \mathrm{kpc}$ of the nuclei of ULIRGs, and only 1 or $2 \AA$ higher at larger radii (Soto \& Martin 2010).

The $\mathrm{H} \alpha$ emission line equivalent widths in our data cover a broad range (from -1 to $-300 \AA$ ), but typically dip below $-10 \AA$ only at radii $\gtrsim 2 \mathrm{kpc}$, and sometimes not at all. Some systems (Mrk 273 and VV 705:NW) have $\mathrm{H} \alpha$ emission line equivalent widths of $\lesssim-20 \AA$ in virtually every spaxel. The equivalent widths vary spatially in a complex way, but generally increase with increasing radius. Furthermore, the Balmer absorption lines in ULIRGs are typically much broader than the emission lines (Soto \& Martin 2010), minimizing their impact on the emission line fitting. The most likely effect of the lack of Balmer absorption correction in our data would be an overestimate of the [N II] $6583 \AA / \mathrm{H} \alpha$ and [O I] $6300 \AA / \mathrm{H} \alpha$ emission line ratios $2-3 \mathrm{kpc}$ from the nucleus in a few systems. Conservatively, this overestimate is no worse than $50 \%$ in those spaxels with emission line equivalent widths near $-10 \AA$. Given the relative widths of the absorption and emission lines, the likely effect is smaller than this. This error does not affect the conclusions of $\oint 5.2$.

The emission lines visible in the wavelength range of our data are $\mathrm{H} \alpha, \quad[\mathrm{N}$ II $]$ 6548, $6583 \AA$, and [O I] $6300,6364 \AA$. We fit 1 - 2 Gaussian velocity components to these emission lines in each spectrum in all cases but Mrk 273, for which we allowed up to 3 components. We fitted a common velocity and FWHM to all emission lines in a given velocity component. The broad emission line component in F08572+3915: NW required a more constrained fit, which is described in $\S 4.1 .2$. The required number of Gaussian components in each spaxel was determined by a combination of software automation and manual inspection. UHSPECFIT discarded all com- ponents that were narrower than the spectral resolution, too broad to be robustly distinguished from the continuum, or fell below $\mathrm{SNR}=3$ in the $\mathrm{H} \alpha$ and [N II] $6583 \AA$ lines. The exception is Mrk 231, for which we required $\mathrm{SNR}>7$ in $\mathrm{H} \alpha$ or [N II] $6583 \AA$.

For spaxels with multiple velocity components, we organize them principally by FWHM into a (1) narrow and (2) broad component (except for Mrk 273; see $\$ 4.4$ ). However, we adjust the classification of some spaxels by hand to achieve continuity in the velocity maps, particularly in cases where the FWHM of the two components are similar.

For five of the six galaxies in our sample, we found a $>3 \sigma$ component in $\mathrm{H} \alpha$ or [N II] $6583 \AA$ in $>90 \%$ of spaxels. In the sixth galaxy, F08572+3915, we detected emission in $>60 \%$ of spaxels.

We fit the Na I D absorption lines using the method of Rupke et al. (2005b). This method robustly separates optical depth and covering factor, even for a blended doublet and multiple velocity components, by using a Gaussian in optical depth. The resulting values for optical depth vary from 0.1 to 5 (the latter is the upper limit of values to which we are sensitive), but are typically of order unity. The covering factor can vary between 0 and 1 , but is typically 0.5 . These results are consistent with previous studies of nuclear spectra of ULIRGs (Heckman et al. 2000, Rupke et al. 2002, 2005b).

One velocity component in Na I D provided good fits to spectra throughout the sample. For most systems, we ignored the nearby He I $5876 \AA$ emission line because of its weakness. The exceptions are VV 705 and Mrk 231; in these cases we fixed the line width and centroid of He I in each spaxel to the values determined by fits to other emission lines in that spaxel.

The stellar contribution to the Na I D absorption line in ULIRGs is generally quite small (Rupke et al. 2005b). In previous studies, the stellar contribution to $\mathrm{Na} I \mathrm{D}$ has been estimated by scaling the strength of $\mathrm{Mg}$ I b (Heckman et al. 2000, Rupke et al. 2002, 2005b). The current data do not probe Mg I b, or indeed other strong stellar features, so we cannot estimate this contribution on a spaxel-by-spaxel basis. For four of the six galaxies in our sample, measurements of nuclear spectra from previous work (Kim et al. 1995; Veilleux et al. 1999a) or from the SDSS allow us to estimate this contribution for at least one nucleus (F08572+3915:NW, F10565+2448, Mrk 273, and VV 705:NW). We find that the equivalent width of $\mathrm{Mg} \mathrm{I}$ b is $1.0 \AA$ or less, yielding a stellar Na I D equivalent width of $0.5 \AA$ or less. Compared to the measured total Na I D equivalent widths in these sources, then, the stellar Na I D represents only $5-10 \%$ of the line.

\subsection{Maps and Velocity Distributions}

The immediate results of our emission- and absorptionline fits to the data are maps of physical properties in each system. The first set of maps for each system (e.g., Figure 1) displays continuum properties: (1) two-color Hubble Space Telescope images (using Advanced Camera for Surveys Wide Field Camera $F 814 W$ and $F 435 W$ data in most cases); (2) continuum flux ratio (color) images using HST data; (3) continuum images from our GMOS data; and (4) the rest-frame equivalent width of $\mathrm{Na} I \mathrm{D}$ in each spaxel. 
The second set of maps (e.g., Figure 3) shows the lineof-sight (LOS) velocity of each emission- and absorptionline component in each spaxel. We display the centroid velocity, the most blueshifted velocity (for the broad components), and the FWHM (with the spectral resolution subtracted in quadrature) of each component in each spaxel. Our line profile modeling is based on Gaussian velocity profiles, so the most blueshifted velocity is defined by the properties of the normal distribution:

$$
\begin{aligned}
& v_{50 \%} \equiv \text { center of Gaussian profile } \\
& \quad(50 \% \text { of gas has lower outflow velocity) } \\
& v_{98 \%} \equiv v_{50 \%}-2 \sigma
\end{aligned}
$$

(98\% of gas has lower outflow velocity)

These definitions assume that negative velocities are blueshifted, and apply to individual Gaussian components in cases where we fit more than one velocity component.

The sole exception to these definitions is Mrk 273. Because Mrk 273 shows significantly redshifted emission as well as blueshifted emission, we define $v_{98 \%}$ for redshifted components in this system by adding, instead of subtracting, $\sigma$.

The third set of maps (e.g., Figure 4 ) presents $\mathrm{H} \alpha$ flux and two emission line flux ratios ([N II] $6583 \AA / \mathrm{H} \alpha$ and [O I] $6300 \AA / \mathrm{H} \alpha$ ) of each component in each spaxel.

The statistical properties of the derived LOS velocity fields are presented in two ways. First, we show histograms of the distributions of all LOS velocities in each system (e.g., Figure 5). Second, we tabulate the mean and extreme values of FWHM, $v_{50 \%}$, and $v_{98 \%}$ in each galaxy with an outflow in Table 3. In the latter, we include only outflowing components in each spaxel.

\subsection{Structural Modeling}

A primary goal of this study is to study the structural properties of the outflows in individual systems. Structural models enable us to better quantify the extent, mass, momentum, and energy in an outflow, and thereby its impact on the galaxy.

Previous surveys of winds in ULIRGs used a spherically symmetric, mass conserving free wind to estimate mass, momentum, and energy in outflows (Heckman et al. 2000, Rupke et al. 2002, 2005c, Soto et al. 2012). These studies made assumptions about the radial extent, radial distribution, and variation of velocity with position of outflows in these systems. Recent IFS studies of outflows in two ULIRGs (Shih \& Rupke 2010, Rupke \& Veilleux 2011) were able to mitigate these uncertainties by providing lower limits on the radial extent of the gas and detailed maps of the gas mass and velocity as a function of position.

In this study, we employ two strategies to model the structure of the outflows in each system. First, we estimate mass, momentum, energy, and their flow rates using a single radius, mass conserving free wind. Next, we use the detailed IFS data to motivate alternative geometries. In particular, we use superbubble models to fit the data for two systems (Mrk 273 and VV 705) that show strong evidence for such structure. Other geometries are possible (e.g., biconical outflows, filled-in geometries instead of shells), but require modeling complexity beyond the scope of the current paper.

\subsubsection{Single Radius Free Wind (SRFW)}

For the Single Radius Free Wind (SRFW) model, we use the time-averaged thin shell formulae of Rupke et al. (2005c), which are derived by assuming a massconserving How. These formulae were modified by Shih \& Rupke (2010) for IFS data by letting each spaxel (corresponding to a unique angular coordinate with respect to the wind's center) have its own mass and velocity. These formulae are equivalent to computing the mass, momentum, and energy at each angular location on the thin shell, and then computing outflow rates by dividing by the dynamical time $t_{d y n} \equiv R_{\text {wind }} / v$ of each spaxel (or angular coordinate). We assume a single radius for the wind in each system, estimated from the maximum extent of the wind in the plane of the sky. Within each system, we use the same wind radius for the ionized and neutral gas phases. We use the velocity measured in each spaxel, deprojected using the assumed radius, as the true wind velocity at that angular location in the wind.

Most of the formulae in Rupke et al. (2005c) and Shih \& Rupke (2010) are expressed in terms of column density, and are in this form only applicable to the neutral gas in our study. However, by computing the ionized gas mass in each spaxel from the $\mathrm{H} \alpha$ luminosity, and dividing by the dynamical time for each spaxel, we can compute momentum, energy, and their outflow rates in each spaxel from their dependence on mass and velocity.

The neutral gas mass in each spaxel is proportional to the $\mathrm{H}$ column density and the deprojected solid angle at that location (i.e., the solid angle subtended by the spaxel with respect to the outflow center). To compute the $\mathrm{H}$ column density, we first find the $\mathrm{Na}$ column density, which depends on the measured optical depth and velocity width in a simple way for our assumption of a Gaussian velocity distribution in each component (see, e.g., the formula in Rupke et al. 2002). This is converted into $\mathrm{H}$ column density using typical Milky Way dust depletion for $\mathrm{Na}$ (-0.95 dex; Savage \& Sembach 1996) and metal abundance of $\mathrm{Na}(-5.69$ dex; Savage \& Sembach 1996), and an uncertain ionization fraction $(y=1-N(\mathrm{Na} \mathrm{I}) / N(\mathrm{Na})=0.9$; Rupke et al. $2005 \mathrm{~b})$. $N(\mathrm{H})$ depends on $y$ as $(1-y)^{-1}$. The solid angle subtended by each spaxel is easily computed in our spherical model, and we then multiply this solid angle by the measured covering factor of the neutral gas, to account for clumpiness in the outflow. (The covering factor in each spaxel equals the fractional area of the spaxel that is covered by absorbing gas, and is a parameter in the absorption line fit; see Rupke et al. $2005 \mathrm{~b}$ for more details.)

The ionized gas mass in each spaxel is computed from the $\mathrm{H} \alpha$ luminosity, assuming Case $\mathrm{B}$ recombination and $T=10^{4} \mathrm{~K}$. It depends on electron density as $n_{e}^{-1}$. We assume $n_{e}=10 \mathrm{~cm}^{-3}$, which is consistent with limits on $n_{e}$ for the superbubble in NGC $3079\left(n_{e}=5-100 \mathrm{~cm}^{-3}\right.$; Veilleux et al. 1994, Cecil et al. 2001).

The assumed radii in the SRFW model for each galaxy with an outflow are listed in Table 4. This table also lists the deprojected covering factor over the surface of the outflow, as seen from the galaxy nucleus, assuming the given radius, and summed over all spaxels with outflowing gas. This integrated covering factor (which we label $\Omega / 4 \pi$ ) represents the fraction of sightlines, for an ob- 
server at the galaxy center, that will encounter the wind. It differs from the opening angle of the wind, which describes the solid angle contained by the outer boundary of the wind. The integrated covering factor is a lower limit to the wind opening angle.

\subsubsection{Bipolar Superbubble (BSB)}

The ionized gas kinematics in Mrk $273(\S 4.4 .3)$ and neutral gas kinematics in VV 705:NW (\$4.5.2) suggest the presence of a Bipolar SuperBubble (BSB). Following similar treatments of other nuclear superbubbles (e.g., Veilleux et al. 1994), we used two prolate ellipsoidal bubbles on either side of the galaxy nucleus. Each bubble has semi-principal axis lengths $R_{z}$ (along the axis shared by the two bubbles) and $R_{x}=R_{y} \equiv R_{x y}$ (perpendicular to the $z$ axis). We assumed that the gas lies on the bubbles' surfaces and that each parcel of gas is moving radially with velocity $v=v_{z}\left(r / r_{z}\right)^{n}$, where $r$ is the distance from the center of the wind, and $v_{z}$ and $r_{z}=2 R_{z}$ are the velocity and distance along the $z$ axis.

For Mrk 273, we optimized seven of the eight BSB parameters $\left(v_{z}, R_{x y}, R_{z}\right.$, line of sight inclination, position angle, and center coordinates) by minimizing the RMS difference between the model and the data. We found that it was not possible to robustly constrain the power law index $n$ beyond the statement that $n \gtrsim 1$ is favored. The best-fit z-axis velocity $v_{z}$ varied with $n$, such that larger values of $n$ favored larger $v_{z}$. However, the best fit values of other parameters varied little or not at all with $n$.

For two different plausible values of $n$ based on studies of other sources (2 and 3; see, e.g., Veilleux et al. 1994 Cecil et al. 2001), we list in Table 5 the best fit BSB parameters for the ionized gas in Mrk 273. In the best fit models, 67 Nyquist-sampled spaxels lie completely inside the bubble edges. However, each spaxel has two outflowing kinematic components (the near and far sides of the bubble), leading to 134 data points in the fit. The RMS difference between the model and data for the redshifted and blueshifted components (with the redshifted and blueshifted RMS values combined in quadrature) is $\sim 200 \mathrm{~km} \mathrm{~s}^{-1}$. We estimated the errors in each parameter by fixing all other parameters and varying the parameter until the RMS increased by $50 \mathrm{~km} \mathrm{~s}^{-1}$.

For VV 705:NW, we performed a similar procedure. Because this disk is almost face-on $(\S 4.5 .2$, any bubble emerging along the minor axis of the disk will also be face on. The outflow in this system was detected primarily in absorption, so we could not detect the far side of the bubble (behind the disk). The resulting 38 distinct data points (in the best fit model) made it infeasible to fit all 8 parameters of the BSB model. Thus, we fixed the center of the outflow to be the NIR luminosity peak $(\$ 4.5 .2$ ). We also constrained the cylindrical radius of the outflow in the plane perpendicular to the $\mathrm{z}$-axis to be $R_{x y}=$ $1.0 \mathrm{kpc}$ after inspecting the extent of the outflow in the plane of the sky. Furthermore, as with Mrk 273 we could only determine that $n \gtrsim 1$. Thus, for $n=2$ and $n=3$ we fit four of the eight $\mathrm{BSB}$ parameters $\left(v_{z}, R_{z}\right.$, line of sight inclination, position angle).

To compute the neutral gas mass in the BSB model, we measured the column density in each spaxel and multiplied by the projected spaxel area. The ionized gas mass was computed as in the SRFW model, and momentum and energy followed from the deprojected velocity of each spaxel. Outflow rates come from dividing by the dynamical time, $2 R_{z} / v_{z}$.

\subsubsection{Results}

The mass, momenta, and energies and their outflow rates derived from the SRFW and BSB models are listed for each system and each gas phase in Table $6^{1}$

Some uncertainties remain in these models. First, there is still LOS uncertainty in the gas position. Second, we employ only "thin shell" models: we assume that the gas is confined to a small radial range $(\Delta R / R<<1)$. Thick shell models are likely to yield smaller masses, momenta, and energies (e.g., Rupke et al. 2005c). Finally, there is uncertainty due to the ionization correction required to convert the amount of $\mathrm{Na}$ I to the total amount of $\mathrm{Na}$ (e.g., Rupke et al. 2002, 2005c). We assume that the Na I represents $10 \%$ of the total Na, based on Galactic sight lines (Rupke et al. 2002). Since $\mathrm{Na}$ resides primarily in gas well-shielded from UV radiation (because of its low ionization potential), the correction is likely not much larger than this.

\section{INDIVIDUAL SYSTEMS}

In this section, we discuss the power source and kinematics of each system in detail.

\section{1. $F 08572+3915$}

This system is a close pair that is likely near second pericenter. The system's energetics are dominated by a buried AGN in the NW nucleus, based on copious evidence. We review this evidence and then discuss the outflow in this system, which shows the highest velocities in our sample. Figures $1-9$ present maps and velocity distributions for each nucleus.

\subsubsection{Power Source}

The NW nucleus is the source of the prodigious farinfrared (FIR) luminosity, as it completely dominates the radio and mid-infrared (MIR) luminosity of the system (Condon et al. 1990; Soifer et al. 2000). Inspection of Spitzer Space Telescope archival images at $3.6 \mu \mathrm{m}$ and $8.0 \mu \mathrm{m}$ shows that the SE nucleus contributes less than $1 \%$ of the system luminosity at these wavelengths. The dominance of the NW source is consistent with the $\sim 2 \times$ $10^{9} M_{\odot}$ of molecular gas detected in the NW nucleus (Evans et al. 2002); only upper limits are obtained in the SE.

Clear evidence for AGN in this system is absent in ground-based optical spectra. Previous optical spectral types of LINER (Veilleux et al. 1999a) or Seyfert 2 (Yuan et al. 2010) in each nucleus were based on shallow spectra that did not significantly detect [O III] $5007 \AA$ or $\mathrm{H} \beta$ (Kim et al. 1998). The SDSS spectrum of the NW nucleus is much deeper than these previous spectra, with a similar spatial footprint. An analysis of the SDSS spectrum using our stellar continuum and emission line fitting

1 We note that the mass, momentum, and energy outflow rates for Mrk 231 are a factor of $\sim 2$ lower than those listed in Rupke \& Veilleux (2011). This is because in Rupke \& Veilleux (2011) we did not multiply the neutral gas quantities in each spaxel by the covering factor of the absorption line gas in that spaxel. In this paper we do include this factor. 
software $\left(\S 2\right.$ yields $A_{V}=2.14$, and a spectral type that is borderline between $\mathrm{H}$ and $\mathrm{C}$ (Kewley et al. 2006). This spectral type is not truly nuclear however, since the line ratios vary significantly in the $\mathrm{E}-\mathrm{W}$ direction across the SDSS aperture. Some of these extended line ratios are consistent with AGN-excited gas, though not uniquely so in the absence of [O III] data (Fig. 4). The optical appearance in $H S T$ images also shows "complex extinction and scattering" (Surace et al. 1998; Fig. 1). In contrast with the NW nucleus, the SE nucleus is clearly of spectral type $\mathrm{H}$ (Fig. 8), even in the absence of [O III] or $\mathrm{H} \beta$ data, consistent with its optical morphology (a "typical galaxy core"; Surace et al. 1998).

In the NW nucleus, NIR recombination lines point to heavier obscuration than in the optical: $A_{V}=6.7$, based on $\mathrm{Pa} \alpha$ and $\mathrm{Br} \gamma$ fluxes (Veilleux et al.1999b) and assuming Case B, $n_{e}=10^{4} \mathrm{~cm}^{-} 2$, and $T=10^{4} \mathrm{~K}$ (Osterbrock 1989). This nucleus is unresolved in the NIR with $H S^{\prime \prime}$, and it becomes more prominent with respect to the extended host between 1.1 and $2.2 \mu \mathrm{m}$ (the half-light radius decreases from $830 \mathrm{pc}$ to $140 \mathrm{pc}$; Scoville et al. 2000). $L$ band and MIR spectra point to a very high $A_{V}$, based on deep absorption features from carbonaceous and silicate dust (Dudley \& Wynn-Williams 1997, Imanishi \& Dudley 2000; Soifer et al. 2002; Armus et al. 2007). 'The MIR source radius is $<130 \mathrm{pc}$ at $12.5 \mu \mathrm{m}$ (Soifer et al. 2000) and $<60 \mathrm{pc}$ at $18 \mu \mathrm{m}$ (Imanishi et al. 2011). The increasing inferred visual extinction and decreasing source size as tracers move to longer wavelengths point to a highly obscured, compact source.

The most likely candidate to power the NW source is a heavily obscured AGN, as confirmed by MIR spectroscopy. The infrared continuum shape and lack of PAH emission provide evidence that a deeply buried AGN powers $\sim 70 \%$ of the total infrared luminosity (Veilleux et al. 2009). Hot dust emission also points to an AGN (Armus et al. 2007). This source is barely detected by the Chandra X-ray Observatory, though its hardness ratio is in principle consistent with heavy obscuration of an AGN (Teng et al. 2009).

\subsubsection{Kinematics}

The SE galaxy reveals modest non-circular motions, indicative of tidal dynamics, superimposed on ordinary rotation of an inclined disk. The line of nodes is uncertain, but the major axis of the bulge is $130^{\circ} \mathrm{E}$ of $\mathrm{N}$. The projected peak-to-peak velocity amplitude along this line is $\sim 150 \mathrm{~km} \mathrm{~s}^{-1}$. The appearance of the spiral arms in the HST image and the redshifted emission in the SE point to the near side of the disk lying in the NE. No absorption-line gas or emission-line outflow is evident in this galaxy.

The narrow ionized gas component in the NW galaxy is undergoing orderly rotation, again with modest noncircular motions mixed in. The central isovelocity contours are not perpendicular to the line of nodes, which we estimate to lie at $120^{\circ} \mathrm{E}$ of $\mathrm{N}$ if it is to pass between the projected rotational maxima $\left( \pm 120 \mathrm{~km} \mathrm{~s}^{-1}\right)$. If the maximum possible disk velocity were $150 \mathrm{~km} \mathrm{~s}^{-1}$ $\left(200 \mathrm{~km} \mathrm{~s}^{-1}\right)$, then the disk inclination would be $53^{\circ}$ $\left(37^{\circ}\right)$. The midpoint of the rotational maxima is offset $\sim 0.5^{\prime \prime} \mathrm{NE}$ of the nucleus. If the tidal features near the nucleus are the elongations of spiral arms, then the near side of the galaxy is to the SW of the nucleus.

The broad emission line component in the NW nucleus of $\mathrm{F} 08572+3915$ required special treatment. We were unable to independently constrain the line fluxes and width of the broad, outflowing gas component in each spaxel because of the large width of this component, which caused severe blending between $\mathrm{H} \alpha$ and [N II]. The outflowing component of the isolated line [O I] was too weak to provide an independent constraint on the line width. We instead used the single aperture Sloan Digital Sky Survey (SDSS) spectrum of the NW nucleus to disentangle the line widths and fluxes of the outflowing component. While our spectrum does not have the wavelength range to include [S II] $6717,6731 \AA$, the SDSS spectrum does, and the outflowing component appears in this line. In both the narrow and broad emission components of the SDSS spectrum, we fit simultaneously all emission lines using a common galactocentric velocity and line width in each component. We constrained the [S II] line ratio to lie within its expected values based on atomic physics. The $\mathrm{H} \alpha$, [N II], and [S II] lines together provided enough leverage to simultaneously fix the line width and line fluxes of the outflowing component. We then used these values to fix the $[\mathrm{N} \mathrm{II}] / \mathrm{H} \alpha$ ratio and line width across the outflow in our data, and we fit only the central velocity and absolute flux in the broad outflowing component in each spaxel. Fig. 10 shows example fits to the broad line in our data.

The broad ionized gas component reveals the highest velocity outflow in our sample, with $\left\langle v_{98 \%}\right\rangle=$ $-2800 \mathrm{~km} \mathrm{~s}^{-1}$ and a maximum value for $v_{98 \%}$ of $3350 \mathrm{~km} \mathrm{~s}^{-1}$. It peaks in velocity directly between the peaks in projected rotational velocity, and shows lower velocities $2 \mathrm{kpc}$ to the S/SW (though still in excess of $\left.v_{98 \%}=-2000 \mathrm{~km} \mathrm{~s}^{-1}\right)$. The blueshifted component is very broad $\left(\mathrm{FWHM}=1500 \mathrm{~km} \mathrm{~s}^{-1}\right)$, but it is not an AGN broad line region because it appears in other forbidden lines ([S II $]$ ) and is spatially resolved.

The neutral outflow in the NW galaxy is located $1-$ $2 \mathrm{kpc}$ SE of the nucleus, and reaches velocities up to $-1150 \mathrm{~km} \mathrm{~s}^{-1}$ (with $\left\langle v_{98 \%}\right\rangle=-900 \mathrm{~km} \mathrm{~s}^{-1}$ ).

The outflow in F08572+3915 is not well enough resolved for a structural model, but the data offer some clues. The emission-line flow extends along the galaxy minor axis, though in a direction behind the near side of the disk. However, at this point in the disk the reddening is low. The decrease of outflow velocity in this direction is consistent with the far-side of a minor-axis outflow. The other direction along the minor axis shows heavy reddening; the outflow is perhaps obscured along this direction. The obscuration along this direction may also indicate a dusty flow. However, no neutral gas is detected here; the neutral gas outflow instead appears SE of the nucleus along the line of nodes. The neutral gas partly overlaps the emission-line outflow, but its overall morphology is unresolved, and it is unclear how the two are related.

\section{2. $F 10565+2448$}

The IFS data for this galaxy, a triple system dominated in luminosity by one member, was first presented in Shih $\&$ Rupke (2010). We review the nature of the power source in this system and revisit the interpretation of the 
IFS data based on a new reduction and analysis with the current data pipeline and a finer binning ( 0 .' 3 spaxels, vs. 0 !'6 in Shih \& Rupke 2010). Figures $11-15$ show maps and velocity distributions for this system.

\subsubsection{Power Source}

Most of the luminosity in this triple, at all wavelengths, arises in the westernmost nucleus. A second and third nucleus are located 8.'0 SE (Scoville et al. 2000) and 25.'8 E (Murphy et al. 1996) of the W nucleus. The E and SE nuclei are not detected in $1.49 \mathrm{GHz}$ VLA images (Condon et al. 1990) or Chandra X-ray images (Iwasawa et al. 2011b). All three nuclei have optical peaks in $R$, with the W nucleus being $2 \times$ as bright as the E nucleus in $2 \mathrm{kpc}$ square apertures (Armus et al. 1990), and the sum of the $\mathrm{W}+\mathrm{SE}$ nuclei being $4 \times$ as bright overall as the $\mathrm{E}$ galaxy (Murphy et al. 1996). The W nucleus also contains 95\% of the system's total $\mathrm{H} \alpha$ flux (uncorrected for extinction; Armus et al. 1990). In the $K$ band, the W+SE nuclei are together $5.6 \times$ as bright as the E nucleus (based on 2MASS $K_{20}$ magnitudes retrieved via NED), and the W nucleus is in turn significantly brighter than the SE nucleus (Murphy et al. 1996).

Judging from Infrared Space Observatory images, the $\mathrm{E}$ nucleus is very faint compared to the $\mathrm{W}+\mathrm{SE}$ complex at 7 and $15 \mu \mathrm{m}$ (Dale et al. 2000). Most of this MIR flux in turn arises from the $\mathrm{W}$ nucleus, as seen in Spitzer archival images at $4-24 \mu \mathrm{m}$. The $\mathrm{W}$ nucleus is the source of the prodigious FIR emission from this system, which is obvious from archival Spitzer 70 and $160 \mu \mathrm{m}$ images.

The E nucleus is a red spheroidal system with no star formation, as evident from HST optical images and the archival SDSS spectrum, which show a smooth light distribution and an absorption-line spectrum. The exact nature of the SE nucleus is unknown, but its blue color is apparent in Figure 11. The W nucleus is classified optically as H II/composite (Veilleux et al. 1995; Yuan et al. 2010), but it shows no high-excitation MIR emission lines (Farrah et al. 2007) or X-ray signatures of an AGN (Teng $\&$ Veilleux 2010; Iwasawa et al. 2011b). Based on PAH strength and MIR spectral shape, Veilleux et al. (2009) determine an AGN fraction of 0.17 .

We conclude that a starburst in the $\mathrm{W}$ nucleus of $\mathrm{F} 10565+2448$ is producing most of the energy in the system and that an AGN, if any is present, contributes very little to this energy output.

\subsubsection{Kinematics}

As discussed in Shih \& Rupke (2010), the ionized gas rotates in the same sense as the molecular gas, in a disk of $\mathrm{PA} \sim 100^{\circ}$ and $i \sim 20^{\circ}$ (Downes \& Solomon 1998). The near side of the disk is not obvious because of the irregular morphology of the galaxy, but, as we discuss below, it is likely to be the $\mathrm{N}$ side.

A neutral outflow was discovered in this system via nuclear spectra (Heckman et al. 2000, Martin 2005; Rupke et al. 2005c), and it is visible in molecular gas, as well (Sturm et al. 2011). Martin (2006) showed that this neutral outflow extends eastward at least $8 \mathrm{kpc}$ from the nucleus, while Shih \& Rupke (2010) showed that its projected extent is at least $4 \mathrm{kpc}$ from the nucleus in most other directions, as well. Shih \& Rupke (2010) found that the neutral gas outflow is dusty and has a velocity profile inconsistent with a symmetric minor-axis outflow.
They concluded that the outflow may be decelerated in the E due to ambient interstellar gas, since significantly higher velocities are observed $\mathrm{W}$ of the nucleus.

We verify the extent and strongly asymmetric velocity structure of the neutral and ionized gas found by Shih \& Rupke (2010). The neutral gas velocity varies slowly with projected galactocentric radius, as we show in Figure 16. At a given position angle ( $\mathrm{E}$ of $\mathrm{N}$ with respect to the galaxy center), these figures show either flat velocity profiles or perhaps a modest decline in $v_{50 \%}$ with projected radius. SRFW models are able to reproduce this behavior as long as the velocity is quite different $\mathrm{E}$ and $\mathrm{W}$ of the nucleus and the wind radius is large enough (at least $5 \mathrm{kpc}$ ). The radial profiles of $v_{98 \%}$ do show a noticeable decline with radius. If both $v_{50 \%}$ and $v_{98 \%}$ represent bulk flow of gas at similar locations in the wind, then this suggests that the gas radius is also not too large $(\lesssim 10 \mathrm{kpc})$. Alternatively, the changes in $v_{98 \%}$ may reflect the decline in unresolved bulk or turbulent motions with radius.

Compared to the analysis of Shih \& Rupke (2010), the current study increases the spatial information available for this source by Nyquist sampling the seeing disk. With 0 .' 3 spaxels, a minor axis outflow on scales of $\sim 1 \mathrm{kpc}$ is evident in the data (Figure 17). The primary evidence for this is in the morphology and extent of the largest FWHM, highest velocity regions in the neutral and ionized gas. If we assume that the blue clumps to the $\mathrm{N}$ of the nucleus represent unobscured star forming regions in the near side of a galaxy disk, then the high velocity regions extend directly $\mathrm{S}$ along the minor axis of the $\mathrm{CO}$ nuclear disk. We would expect blueshifted emission on the $\mathrm{S}$ side of the disk, as is observed. The angle that the wind makes with respect to the line of sight should be near that of the disk's inclination $\left(\sim 20^{\circ}\right.$; Downes \& Solomon 1998). The minor-axis flow must then be relatively collimated (total opening angle, from one edge of the wind to the other, of $\lesssim 2 \times 20^{\circ}$ ), since we observe it primarily in projection away from the line of sight.

The regions of highest velocity are strikingly similar in morphology to the dustiest regions of the disk, if we assume that the HST color just $\mathrm{S}$ of the nucleus is due to variable obscuration (consistent with the patchy and filamentary structures of the colors in this region). The largest values of FWHM in the gas (up to $800 \mathrm{~km} \mathrm{~s}^{-1}$ ) are coincident with the dustiest region of the galaxy (and region of highest $\mathrm{Na}$ I D equivalent width). Dust filaments extend $\mathrm{S}$ along the minor axis, coincident with regions of high velocity, suggesting that dust is being entrained in the wind along this direction.

\subsection{Mrk 231}

Mrk 231 is a late stage merger in which the nuclei have already coalesced (Surace et al. 1998, Veilleux et al. 2002). It is the nearest QSO (Boksenberg et al. 1977), as well as the nearest broad absorption line (BAL) QSO Adams \& Weedman 1972). The kpc-scale neutral outflow in this system was discovered by Rupke et al. (2005a), and was more recently found to extend in all directions (Rupke \& Veilleux 2011) and have a significant molecular component (Fischer et al. 2010a; Feruglio et al. 2010; Aalto et al. 2012; Cicone et al. 2012).

Mrk 231 is dominated by a central point source $(3 \times$ brighter than the host at $H$; Veilleux et al. 2006) that 
has a QSO spectrum with broad Balmer and Fe lines (Boksenberg et al. 1977). It also hosts a massive CO disk (Downes \& Solomon 1998) with a high star formation rate, based on the presence of $\mathrm{PAH}$ emission, [Ne II] $12.81 \mu \mathrm{m}$ emission beyond that required for an AGN, and MIR colors (Genzel et al. 1998, Veilleux et al. $2009)$. The AGN produces $\sim 70 \%$ of the galaxy's luminosity (Veilleux et al. 2009).

The kinematics of the disk rotation and outflow in Mrk 231 are discussed in Rupke \& Veilleux (2011). We present the entirety of the Mrk 231 data set here for easy comparison with the rest of our sample, but do not discuss it further. Figures $18-21$ show maps and velocity distributions for this system.

\subsection{Mrk 273}

Mrk 273 is a late merger with a binary nucleus and an AGN. The bolometric luminosity of the galaxy is probably dominated by a starburst, but the AGN contribution is still significant. The kinematics in this system are complicated, but clearly show a high-velocity outflow. Maps of derived properties and velocity distributions can be found in Figures $22,26$.

\subsubsection{Power Source}

The nuclear properties of Mrk 273 are enigmatic. Two nuclei (typically labeled $\mathrm{N}$ and SW) are evident in the NIR and MIR (Majewski et al. 1993, Knapen et al. 1997. Scoville et al. 2000; Soifer et al. 2000, see Figure 23). The $\mathrm{N}$ nucleus contains most of the molecular gas in the system within an extremely compact core (radius $\lesssim$ 120 pc; Downes \& Solomon 1998), and is the source of most of the MIR luminosity (Soifer et al. 2000). In the radio, Mrk 273 resolves into a diffuse radio source with two primary peaks (Carilli \& Taylor 2000). The $\mathrm{N}$ radio peak (coincident with the $\mathrm{N}$ nucleus) is further resolved as multiple sources at the highest resolutions, and the SE radio peak is inconsistent with an AGN based on spectral slope (Bondi et al. 2005). The optical line emission from the center of Mrk 273 classifies it as a Seyfert 2 (Koski 1978 Veilleux et al. 1999a). Using IFS, Colina et al. (1999) locate the Seyfert 2 emission near, but to the SW of, the two nuclei.

Recently, the SW nucleus has been identified as an AGN through localization of the hard X-ray point source (Iwasawa et al. 2011b), which is Compton thick based on spectral modeling from $2-40 \mathrm{keV}$ (Teng et al. 2009). The AGN shows strong high-ionization narrow-line emission in the NIR and MIR: possible [Si VI] (Veilleux et al. 1999b) and strong $[\mathrm{Ne} \mathrm{V}]$ and $[\mathrm{O}$ IV $]$ (Genzel et al. 1998, Armus et al. 2007; Veilleux et al. 2009). The ratios of these high-ionization lines to a low-ionization line, [Ne II], point to a dominant contribution of the AGN to the FIR luminosity (Veilleux et al. 2009). Combined $\mathrm{PAH}$ and emission-line diagnostics suggest that the starburst and AGN contribute similarly (Genzel et al. 1998 Veilleux et al. 2009), while MIR continuum diagnostics point to a dominant starburst (Veilleux et al. 2009).

NIR IFS of the inner kiloparsec of Mrk 273 localizes high-ionization coronal line emission ([Si VI]) to the SW nucleus and the SE radio source (U et al. 2012). These new data suggest that the $\mathrm{N}$ nucleus may harbor a heavily-obscured AGN (see also Iwasawa et al. 2011b) that photoionizes the gas in the SE radio source. Alternatively, high-velocity shocks may produce this emission. Regardless, the existence of an obscured AGN in the $\mathrm{N}$ nucleus remains to be investigated.

The concentration of $\mathrm{CO}$ and MIR continuum emission in the northern nucleus would seem to imply that the SW AGN contributes little to the bolometric luminosity of this dusty system. However, MIR dianostics suggest otherwise, with an average AGN fraction of 0.46 (Veilleux et al. 2009). This conclusion relies on the combined MIR spectrum of both nuclei, and rests primarily on the high emission-line ratios. The bolometric AGN luminosity inferred this way is entirely consistent with the X-ray emission of the SW nucleus if we compare it to opticallyselected QSOs. If the AGN luminosity were lower by more than a factor of $2-3$, then $L_{2-10} \mathrm{keV} / L_{A G N}$ would be too high (Teng \& Veilleux 2010).

\subsubsection{Basic Kinematics}

The gas kinematics in Mrk 273 are the most complex in our sample. The atomic and molecular gas kinematics centered around the $\mathrm{N}$ nucleus are characterized at 100 pc to kpc scales by a rotating disk of gas (Downes \& Solomon 1998, Cole et al. 1999; Carilli \& Taylor 2000; Yates et al. 2000). The position angle of the line of nodes of this disk is $70-90^{\circ}$, and it is inclined by $45-55^{\circ}$ with respect to the line of sight (Downes \& Solomon 1998, Cole et al. 1999; U et al. 2012). 'The disk is rotating rapidly, with a projected velocity gradient of $400-500 \mathrm{~km} \mathrm{~s}^{-1}$ over a few hundred pc (velocity increasing from east to west; Downes \& Solomon 1998, Cole et al. 1999, Carilli \& Taylor 2000; Yates et al. 2000). On larger scales, there is a molecular feature that extends $5 \mathrm{kpc} \mathrm{N}$ and one that extends $5 \mathrm{kpc} \mathrm{S}$ of the $\mathrm{N}$ nucleus; the $\mathrm{N}$ feature is redshifted by $\sim 300 \mathrm{~km} \mathrm{~s}^{-1}$, and the $\mathrm{S}$ feature is near systemic (Downes \& Solomon 1998).

There are significant departures from simple E-W rotation, from sub-kpc to kpc scales. The spatially resolved CO lines are broad, and the integrated profile spans $1000 \mathrm{~km} \mathrm{~s}^{-1}$ (Downes \& Solomon 1998). Two components emerge in the [O III] $5007 \AA$ spectra of Colina et al. (1999), and they decompose these into a systemic component and a component that is redshifted in the $\mathrm{E}$ and blueshifted in the $\mathrm{W}$, with a peak-to-peak amplitude of $2400 \mathrm{~km} \mathrm{~s}^{-1}$. They suggest that the latter component represents a starburst-driven superwind.

The present IFS data represent a significant improvement in sensitivity, spatial resolution, and spectral resolution over the Colina et al. (1999) dataset, as well adding information on the neutral gas phase.

It should be noted that the $\mathrm{CO} / \mathrm{H}$ I gas disk is tiny compared to the FOV of our maps, subtending only $1-$ 2 GMOS spaxels (Downes \& Solomon 1998; Carilli \& Taylor 2000).

The ionized gas profiles are extremely broad in many spaxels, and are in most cases best fit by two or three components. Fig. 27 shows several examples of these fits, and Fig. 28 shows the number of components in each spaxel. We order these components into one of four categories as follows: (1) rotating, (2) broad and redshifted, (3) broad and blueshifted, and (4) isolated clouds with narrow profiles. In a two-component spaxel, we assume the narrower component is rotating, though we tweak 
some spaxels by hand because the FWHM of the two components are similar. The second component is then assigned to the broad and redshifted bin or broad and blueshifted bin depending on its velocity with respect to the rotating component. The isolated clouds with narrow line profiles are easily distinguished from the rotating narrow component because of the significant red- or blueshifts of the isolated clouds, and from broad components by linewidth and spatial footprint. If there are three components in a spaxel, we generally choose the central component to be rotating, though there are some exceptions.

The rotating ionized component follows the $\mathrm{CO}(1-0)$ rotation curve of Downes \& Solomon (1998) very closely, including the twisting of the systemic isovelocity contour, the peak redshifted velocity just $\mathrm{W}$ of the nuclei, and the redshifted gas to the $\mathrm{N}$. The rotating component is on average narrow compared to the other components $\left(\langle\mathrm{FWHM}\rangle=300 \mathrm{~km} \mathrm{~s}^{-1}\right)$. The line ratios are low along a NNE/SSW axis, with $\log ([\mathrm{N} \mathrm{II}] / \mathrm{H} \alpha) \sim-0.6-0$ and $\log ([\mathrm{O} \mathrm{I}] / \mathrm{H} \alpha)$ in the range -1.8 to -0.8 . These ratios increase to 0.3 and -0.5 to the ESE and WNW.

The redshifted and blueshifted broad, ionized components are broadly consistent with Colina et al. (1999): the blueshifted components lie primarily $\mathrm{W}$ of the nuclei, while the redshifted components lie to the E. However, our higher spatial and spectral resolution shows significant substructure. Projected velocities rise away from the nucleus, peaking at a remarkable $v_{50 \%}=$ $-1500 \mathrm{~km} \mathrm{~s}^{-1}, 4-5 \mathrm{kpc} \mathrm{NW}$ of the nuclei. However, the broadest components (FWHM up to $1500 \mathrm{~km} \mathrm{~s}^{-1}$ ) with the highest $[\mathrm{N} \mathrm{II}] / \mathrm{H} \alpha$ and $[\mathrm{O} \mathrm{I}] / \mathrm{H} \alpha$ line ratios (indicative of shock ionization) arise directly $\mathrm{N}$ and $\mathrm{S}$ of the nuclei. (These locations also display modest peaks in $\left|v_{50 \%}\right|$ of $\sim 1000 \mathrm{~km} \mathrm{~s}^{-1}$.)

The neutral gas in Mrk 273, as probed by Na I D, is backlit by the stellar continuum across most of the N-S extent of the GMOS FOV in a band that is $1-2 \mathrm{kpc}$ wide. There are few departures from velocities near systemic. However, there is blueshifted gas north of the nuclei. In the SRFW model calculations for this system, we include only spaxels in which the neutral gas is blueshifted from the ionized gas narrow component in that spaxel by more than $50 \mathrm{~km} \mathrm{~s}^{-1}$.

The blueshifted ionized and neutral gas in the N/NW of the system is clearly outflowing, as is the redshifted ionized gas in the S/SE. The velocities (reaching $1500 \mathrm{~km} \mathrm{~s}^{-1}$ in projection) are too high to be explained simply by tidal motions, and are much larger than the projected velocities in the narrow component (between -350 and $350 \mathrm{~km} \mathrm{~s}^{-1}$ ). However, the redshifted and blueshifted ionized and neutral components in other locations have more modest velocities $\left(v_{50 \%}\right.$ and FWHM of a few hundred $\mathrm{km} \mathrm{s}^{-1}$ ) that could in principle be explained by tidal motions.

The evidence for a bipolar outflow aligned E-W, as proposed by Colina et al. (1999), is not the most likely explanation based on the current data, though a variation on this theme emerges from our analysis. The data conclusively point to a bipolar nuclear superbubble in the center of the system, aligned N-S. Gas further from the nucleus, if it is indeed outflowing, then traces gas blown out from this bubble or earlier ejections from the nuclei.

\subsubsection{Nuclear Superbubble}

Position-velocity (PV) diagrams of the line emission in the center of Mrk 273 reveal the classic signature of a bipolar superbubble (Figure 29). Applying our BSB model ( $\S 3.4 .2$ ) and optimizing its parameters yields the values given in Table 5. The model is overplotted in the PV diagrams, and the two-dimensional projected velocity fields and bubble shapes are compared to the data in Figure 30. The bubble is fairly symmetric in emission $\left(R_{x y}=0.8 R_{z}\right)$.

The absorption lines in Mrk 273 are not of high enough signal-to-noise near the possible superbubble to accurately parameterize its neutral gas content. There are higher absorption line velocities near the edges of the near-side bubble in the $\mathrm{N}$, suggesting that some neutral material is entrained by an expanding bubble.

Overall, a bubble shape fits the inner radii of the wind well (at projected radii $\lesssim 2 \mathrm{kpc}$ ). However, the high velocities seen immediately adjacent to it but at larger radii in the NW and SE strongly suggest that the bubble is broken on its surface and gas is escaping the bubble in some directions. This gas has as high or higher velocities than in the bubble itself, perhaps indicating further acceleration by a hot medium that could be blowing the bubble.

There are also areas of high velocity directly $\mathrm{E}$ and $\mathrm{W}$ of the nucleus, along a position angle perpendicular to the bubble axis. These may represent earlier episodes of gas ejection, or ejection due to an unrelated process.

The fitted outflow center is formally located between the two nuclei, 0 .'4 ( $\sim 300 \mathrm{pc}$ ) from the $\mathrm{N}$ nucleus and $0{ }^{\prime \prime} .7$ $(\sim 500 \mathrm{pc})$ from the SW nucleus. However, the errors in the bubble center are 1 spaxel $\left(0{ }^{\prime \prime} 3\right)$, so an origin at one or the other of the two nuclei is plausible. If in fact the outflow arises between the two nuclei, then it could be powered by a combination of energy from the AGN and starburst. However, the outflow may also be asymmetric (extending further on the near or far side due to, e.g., asymmetric acceleration), lending further uncertainty to the true outflow center.

If the bubbles were centered on the $\mathrm{N}$ nucleus, their position angle $\left(-5^{\circ}\right)$ would be consistent with collimation by the tilted $\mathrm{CO} / \mathrm{H}$ I disk at that location (PA $70-90^{\circ}$; Downes \& Solomon 1998; Cole et al. 1999). The disk's inclination is $i=45^{\circ}$ from CO modeling (Downes \& Solomon 1998), which is inconsistent with the superbubble inclination of $75 \pm 5^{\circ}$. However, a higher disk inclination is not out of the question, given the large velocity gradients in the nucleus (Cole et al. 1999, Carilli \& Taylor 2000, Yates et al. 2000). A more recent analysis of NIR IFS data, taken with adaptive optics, finds a more inclined disk $\left(i \sim 55^{\circ}\right)$ based on ionized gas lines (U et al. 2012).

The fitted outflow center is further from the SW nucleus. If the bubbles were centered on the SW nucleus, it is unclear what would collimate the outflow. Furthermore, the $\mathrm{N}$ disk has a large gas reservoir to couple with the energy driving the outflow, while the SE nucleus does not (Downes \& Solomon 1998; Cole et al. 1999, Carilli \& Taylor 2000).

Our structural analysis is inconclusive on the power source of the entire outflow (superbubbles + extended flow), but more consistent with the superbubbles being 
powered by the $\mathrm{N}$ nucleus than the SW nucleus. Data of higher spatial resolution are needed, as well as constraints on the presence of an obscured AGN in the $\mathrm{N}$ nucleus. As we discuss below, however, the high velocities suggest an AGN-driven flow ( $(5.4 .3$.

\section{5. $V V 705$}

$\mathrm{VV} 705$ is a binary merger with nuclear separation of $7.5 \mathrm{kpc}$, based on inspection of an archival HST Wide Field Camera 3 F160W image. Maps of derived properties and velocity distributions for each nucleus are shown in Figures $31-40$. The superbubble emerging from the NW nucleus is one of two in our sample.

\subsubsection{Power Source}

The NW nucleus contains most of the luminosity in this system at multiple wavelengths: $8.44 \mathrm{GHz}$ (NW:SE luminosity ratio of $3: 1$; Condon et al. 1991), $2-10 \mathrm{keV}$ (8:1; Iwasawa et al. 2011a), and $4-24 \mu \mathrm{m}$ (archival Spitzer IRAC and MIPS $24 \mu \mathrm{m}$ data). Though exact measurements are not available, at least 90\% (and perhaps closer to $\sim 99 \%$ ) of the $24 \mu \mathrm{m}$ emission emerges from the NW nucleus, based on the strength of the $24 \mu \mathrm{m}$ PSF of the NW nucleus. This strongly suggests that this nucleus is the source of most of the FIR luminosity of the galaxy, as well. This would be consistent with it hosting most of the molecular gas $\left(\sim 10^{10} M_{\odot}\right.$; Sanders et al. 1991; Chini et al. 1992), based on the narrow FWHM of CO (100 $\mathrm{km} \mathrm{s}^{-1}$; Chini et al. 1992) and the measured $\mathrm{CO}$ velocity near that of the NW nucleus $\left(c z=12070-12090 \mathrm{~km} \mathrm{~s}^{-1}\right.$; Chini et al. 1992; Yao et al. 2003 and this work).

Both nuclei are classified as composite by Yuan et al. (2010), based on spectra obtained by Kim et al. (1995). This is confirmed for the NW nucleus by nuclear data from Moustakas \& Kennicutt (2006). However, there is no evidence for an AGN in either nucleus; X-ray imaging shows soft spectra in both nuclei (Iwasawa et al. 2011a), MIR spectra reveal no $[\mathrm{Ne} \mathrm{V}]$ or $[\mathrm{O}$ IV $]$ fine structure lines (Dudik et al. 2009), and compact radio emission is consistent with a nuclear starburst (Smith et al. 1998).

In the absence of evidence for an AGN, we conclude that any outflows in this system will be powered by star formation. We assume an AGN fraction of $10 \%$ for consistency with the other two galaxies in our sample without luminous AGN, but the actual fraction could be lower.

\subsubsection{Kinematics}

The SE nucleus shows ordered rotation with noncircular motions superimposed. We estimate a line of nodes PA of $-30^{\circ}$. The peak-to-peak velocity amplitude along this PA reaches $250-300 \mathrm{~km} \mathrm{~s}^{-1}$. The near side of the disk is likely to the $\mathrm{W}$, given that this side of the nucleus is less obscured (Figure 36). The line of nodes and rotation amplitude of the $\mathrm{NW}$ nucleus are more difficult to quantify due to tidal motions and a near face-on orientation. We do not attempt to estimate a PA or inclination for this nucleus.

Non-circular motions and multiple narrow components in the SE nucleus include a region of LOS overlap between the two gas disks, $3 \mathrm{kpc} N$ of the SE nucleus. Multiple components are also evident within $1 \mathrm{kpc}$ of the SE nucleus; some of these components have FWHM exceeding $600 \mathrm{~km} \mathrm{~s}^{-1}$. These broad components have velocities within $100-200 \mathrm{~km} \mathrm{~s}^{-1}$ of the narrow component in the same spaxel. They may arise in a weak outflow along the minor axis and/or with infalling shocked tidal debris. Either of these interpretations are consistent with the observed elevated FWHM and line ratios, but the data is not sufficient to distinguish between these possibilities. Regardless, any outflow in this nucleus is not powered by the primary source of luminosity in the merger.

The NW nucleus shows evidence for a superbubble emerging almost directly along the line of sight. This bubble is most evident in absorption, as seen in the PV diagram in Figure 41. Applying our BSB model (\$3.4.2 and optimizing its parameters yields the values given in Table 5. The fits and residuals in two dimensions are shown in Figure 42 .

The superbubble model fits the inner $2 \mathrm{kpc}$ well, but outflowing gas at lower velocities is also visible $\mathrm{NE}$ of the bubble and, to a lesser extent, to its SW. The bestfit parameters show that the outflow emerges almost directly along the line of sight and reaches a velocity of $v_{50 \%}=-500$ to $-600 \mathrm{~km} \mathrm{~s}^{-1}$ along the bubble axis. The elongation of the bubble is fairly uncertain, and is consistent with either a symmetric bubble $(\sim 2 \mathrm{kpc}$ in diameter) or one that is longer perpendicular to the galaxy disk by factors of $2-3$.

The outflow is also seen in emission (Figure 33). However, the ionized gas only shows acceleration in a fraction of the outflow area that is seen in absorption. The broad component in most spaxels has the same velocity as the narrow, rotating component. As a result, in the SRFW model calculations for this system, we include only spaxels in which the broad component is blueshifted from the narrow component in that spaxel by more than $50 \mathrm{~km} \mathrm{~s}^{-1}$.

\subsection{F17207-0014}

This system is a coalesced merger with a diffuse, patchy optical morphology. We present maps and velocity distributions based on our GMOS data in Figures $43-47$ F17207-0014 is the only system in our sample without a detected ionized outflow, though it does reveal a large scale neutral gas outflow.

\subsubsection{Power Source}

All of the data on this system is consistent with an obscured starburst. The nucleus is obscured in the optical but resolved in the NIR (Murphy et al. 1996; Scoville et al. 2000, Figure 44). It is a diffuse source in the radio and MIR (Soifer et al. 2000, Momjian et al. 2003, Baan \& Klöckner 2006), no $|\mathrm{Ne} \mathrm{V}|$ or $\mid \mathrm{O}$ IV $]$ emission is detected in the MIR (Farrah et al. 2007), the AGN fraction based on MIR diagnostics is only 0.11 (Veilleux et al. 2009), and the X-ray data do not show an obscured AGN (e.g., Franceschini et al. 2003, Teng \& Veilleux 2010; Iwasawa et al. $2011 b)$.

\subsubsection{Kinematics}

The CO and H I disk in F17207-0014 subtends $1-2^{\prime \prime}$ along a line of nodes position angle of $\sim 120^{\circ}$ (Downes \& Solomon 1998, Momjian et al. 2003). Arribas \& Colina (2003) presented ionized gas maps of this system and 
showed that the molecular and neutral kinematics are seen in the ionized gas, as well. The rotation of the nuclear ionized disk is roughly centered on the NIR peak, but at larger scales the isovelocity contours twist away from this pattern (Arribas \& Colina 2003). The kinematics on galactocentric radii $>1 \mathrm{kpc}$ probably reflect tidal motions (Arribas \& Colina 2003).

A neutral outflow in this system was discovered by Rupke et al. (2005c) and Martin (2005), and shown to extend over 5-6 kpc (Rupke et al. 2005c , Martin 2006). Martin (2006) found no variation in the outflow velocity (with respect to the rotating ionized gas) along a position angle of $170^{\circ}$. Recently, Westmoquette et al. (2012) presented ionized gas maps of F17207-0014 that showed two velocity components in the ionized gas in the center of this system. However, the two components have very similar velocity, and are not obviously an ionized outflow.

Our new maps of the ionized and neutral gas in F17207-0014 improve on the Arribas \& Colina (2003) and Westmoquette et al. (2012) studies by adding the neutral gas kinematics and increasing the spatial resolution. Our ionized gas maps indeed resemble higher resolution versions of the Arribas \& Colina (2003) maps. We do not detect two ionized gas components, as in Westmoquette et al. (2012), but the much larger spaxels in the Westmoquette et al. (2012) data may increase their sensitivity.

We detect extended neutral gas that is everywhere outflowing in this system, consistent with previous nuclear and extended detections. However, we show that this outflow extends in all directions, and to galactocentric radii of at least $4 \mathrm{kpc}$ (with a maximum extent of $6 \mathrm{kpc}$, as in Martin 2006)

Contrary to Martin (2006), we find that the outflow velocity increases with increasing projected radius (Figure 48). This is inconsistent with our fiducial SRFW model, which predicts decreasing velocity with increasing projected radius. An alternative scenario is that the projected galactocentric radii represent the true galactocentric radii, and that the outflow speed is increasing with radius.

\section{SAMPLE TRENDS}

Our sample of six merging systems reveals an extended outflow in every case. In five systems there is an ionized outflow (F17207-0014 is the odd galaxy out), and four of these are new ionized outflow detections (the Mrk 273 outflow was previously known; Colina et al. 1999). In six systems there is a neutral outflow. Three of these are new detections (F08572+3915, Mrk 273, and VV 705), despite two of these three having been observed in neutral outflow surveys using single-aperture spectroscopy.

In this section we combine the results from our sixgalaxy sample to draw broader conclusions about the multiphase structure and power sources of galactic winds in ULIRGs. These conclusions are summarized in $\S 6$ and rely in part on the analysis in Figures $49-56$

\subsection{Wind Structure}

\subsubsection{Ionized Gas}

We know a great deal about the structure of winds in local disk galaxies hosting starburst-driven superwinds (Veilleux et al. 2005). These winds typically emerge along the minor axis of the disk as either a bipolar superbubble or a bicone. The ionized gas in such BSBs or bicones is often limb-brightened and filamentary, suggesting it lies on the edges of the bubble or cone (e.g., Heckman et al. 1987; Bland \& Tully 1988; Veilleux et al. 1994 Martin 1998, among many examples). In the model of a bipolar wind, these bubbles or cones have opening angles that increase with radius, reaching angles (on one side of the disk) of $45-100^{\circ}$ (Veilleux et al. 2005).

The structure of ionized gas outflows in local mergers is less understood, though the two nearest and moststudied examples have possible minor-axis and/or bipolar flows: Arp 220 (Heckman et al. 1987) and NGC 6240 (Veilleux et al. 2003; Bush et al. 2008). The kinematics of the ionized gas in mergers are made complex by the prevalence of multiple overlapping disks and tidal motions (Colina et al. 2005), as well as outflows. However, careful spectral decomposition of the emission lines is able to separate gas rotation from other motions (Shih \& Rupke 2010, Rupke \& Veilleux 2011; Westmoquette et al. 2012, and this study). The narrow component in the ionized gas (always the strongest in flux overall) follows the rotation of the resolved kpc-scale CO disk (when $\mathrm{CO}$ has been previously observed via interferometry at arcsecond resolution, as in $4 / 6$ systems in our sample). The good match between the ionized and molecular gas in the inner $1-2 \mathrm{kpc}$ of these systems is somewhat surprising, since these disks are dusty and probably optically thick (Downes \& Solomon 1998); thus, the ionized gas is likely to trace the outer "skin" of the disk rather than its core, or emerges from a patchy and inhomogeneous medium. Where the molecular gas is more extended (e.g., Mrk 273), there continues to be a good correspondence between the bulk motions of the molecular and ionized gas.

The linewidths in the narrow component of the ionized gas are relatively large (the average FWHM in this component in each of the eight nuclei in our sample is in the range $100-300 \mathrm{~km} \mathrm{~s}^{-1}$, and typically near $200 \mathrm{~km} \mathrm{~s}^{-1}$; see, e.g., Figure 5). Similar linewidths are seen in the resolved molecular gas in these systems (Downes \& Solomon 1998). These large linewidths may result from several effects. They do not likely result from beam smearing, since the narrow component velocity fields typically have smaller amplitudes than this. Sometimes overlapping disks and/or regions where two disks are colliding (as in F08572+3915 and VV 705:NW) amplify the linewidths in isolated regions as a result of the combined motion of the two disks or the tidal motions of collision. In other systems, the linewidth may be inflated by either energy injected by star formation and/or AGN activity in the inner disks, or on larger scales by tidal motions resulting from the ongoing merger.

On scales larger than the central $1-2 \mathrm{kpc}$, the ionized gas continues the rotational pattern of the inner disks, but often with isovelocity twists superimposed or changes in velocity amplitude. It is likely that the motions of the ionized gas on large scales in most of these systems better reflect the ongoing merger of the two initial systems rather than the inner disk of either nuclei or the nuclear disk of the coalesced system. The ionized gas motions at projected galactocentric radii of $3-5 \mathrm{kpc}$ thus result from a combination of the internal angular momenta of the original two systems with that of the merger orbit. 
The interpretation of the broader and blueshifted or redshifted velocity components in the ionized gas is relatively straightforward. In emission and without other information, outflow and inflow are in principle indistinguishable. However, as we have shown for Mrk 273 and VV 705, outflows can be distinguished from inflow via structural modeling, since BSBs are obviously present. A multiphase, multi-kpc minor axis outflow is also likely present in F10565+2448, based on the morphological connections between the ionized gas kinematics, neutral gas kinematics, and nuclear dust filaments. In the cases of F08572+3915, the large-scale kinematics in Mrk 273, and the inner $\mathrm{kpc}$ of Mrk 231, the observed velocities on these scales are simply too high to be explained by tidally-induced inflow.

Soto et al. (2012) also argue that, in ULIRGs, the observed ionized gas must be on the near side of the galaxy, and thus outflowing if blueshifted, since far-side ionized gas would be obscured by dust. There is some evidence from our data against this conclusion, but there is also evidence to support it; the reality may be more nuanced. First, the continuum obscuration by dust (as revealed by multicolor HST imaging; e.g., Figure 17) is patchy. Nonetheless, optically thick molecular disks (not traced by the optical continuum) reside at the nuclei of these systems and probably obscure any far side ionized gas very near the nucleus. Second, we do observe the receding side of the superbubble in Mrk 273. However, this bubble is highly inclined and extended; in most other cases where we see evidence consistent with a near-side minor axis ionized flow, evidence for the far-side flow is weaker or absent (see discussion below and of individual objects above). The good correspondence between ionized and neutral gas velocities (\$5.3) also suggests that the ionized gas is primariliy located on the near side of these systems, where the absorbing gas must lie. Overall, it is clear that spatially resolved observations of these systems, as we present here, are crucial to resolve these structural degeneracies.

The ionized outflows we observe are in all cases consistent with being collimated by a molecular disk within the galaxy, emerging along the minor axis of that disk. In the three cases where CO disks and ionized outflows are observed (F10565+2448, Mrk 231, and Mrk 273), there are outflows reaching $1-2 \mathrm{kpc}$ away from the nucleus that have directions and inclinations consistent with the $\mathrm{CO}$ minor axis. In the other two cases of ionized outflows where CO disks are present (but have not been observed via interferometry), the direction and inclination of the outflows are consistent with the disk minor axis as inferred from the rotation of the ionized gas.

In particular, in the two cases where we fit the 3D shape of the outflow, the kinematics are consistent with $2 \mathrm{kpc}$ diameter BSBs, which would formally have (onesided) opening angles of $180^{\circ}$. In reality, the footprint of the outflow is not a point, such that the opening angle is smaller than this. For Mrk 273, let us assume the CO core/disk edges from Downes \& Solomon (1998) as the extent of the launching region (radii $100-400 \mathrm{kpc}$, depending on the measure). If we draw a line connecting the $\mathrm{CO}$ disk edge with the bubble edge at a disk height of $1 \mathrm{kpc}$, then we infer an opening angle of $30-40^{\circ}$, similar to local superbubbles.

Outflowing ionized gas is also observed away from the minor axis, particularly in the cases of Mrk 273 and F10565+2448. The morphology of these extended flows is more ambiguous, though in Mrk 273 it may in part be breakout from the superbubble $(\S 4.4 .3)$. It is possible that these are traces of earlier bubbles or ejection episodes that have moved to larger radii.

This idea is buttressed by the timescale for wind evolution over the galactocentric radii probed by our observations, which is $t_{d y n} \sim R_{\text {wind }} / v_{50 \%}=10 \mathrm{Myr}$ (for $R_{\text {wind }}=3 \mathrm{kpc}$ and $\left.v_{50 \%}=300 \mathrm{~km} \mathrm{~s}^{-1}\right)$. The timescale for the orbital evolution of the galaxies in our sample is of order $\gtrsim 100$ Myr (e.g., Barnes \& Hernquist 1996, Mihos \& Hernquist 1996); i.e., an order of magnitude larger. Thus, if we assume that we are observing the six galaxies in our sample at a random time late in the merger, then the existence of minor axis flows at roughly similar scales $(1-2 \mathrm{kpc}$ projected radii) in each system suggests that they are a relatively continuous phenomenon, either in the form of multiple ejection episodes or a continuous flow. This in turn further suggests that the removal of gas from the nuclei of mergers is an efficient process. We return to this subject in $\oint 5.5$.

\subsubsection{Neutral Gas}

The prevalence of minor-axis outflows collimated by a $\mathrm{CO}$ disk is seen in neutral, as well as ionized, gas. F10565+2448, Mrk 231, Mrk 273, and VV 705:NW all show evidence of expanding neutral flows along the minor axis, roughly consistent with the morphology of the ionized flows. In the neutral gas, however, there are exceptions: F08572+3915 and F17207-0014 do not obviously show flows along the $\mathrm{CO}$ minor axis, despite revealing outflowing neutral gas at other locations. Furthermore, in F10565+2448 and VV 705:NW, there are extensive areas of outflowing neutral gas away from the minor axis flow.

In the case of neutral gas, there is structural ambiguity for two reasons: (1) the neutral gas requires a strong continuum background source, so the absorptionline signal-to-noise ratio decreases away from the galaxy nucleus; and (2) the Na I D resonant line may suffer from emission-line filling near systemic (Prochaska et al. 2011). We try to minimize the former by deep exposures, but there is still a limiting radius at which the signal drops below detectability. Though we cannot provide quantitative constraints, we believe the latter to be minimal, since the strong dust obscuration in these systems would prevent outflowing emission from the far side of the outflow from reaching us (see discussion in $\$ 5.1 .1$ for more on far-side line emission). This qualitative conclusion is supported by the lack of any observed redshifted $\mathrm{Na}$ I D emission in these systems, even at large radii where the dust obscuration is smaller than in the nuclei.

\subsubsection{Maximum Extent and Opening Angle}

The extended outflows in our dataset are consistently large, stretching across much of the FOV. Five of the six systems in our sample show outflowing gas up to the edge of the FOV, at scales ranging from $3-5 \mathrm{kpc}$ from the nucleus. Thus, the sizes inferred from our data are ultimately lower limits to the overall wind sizes in these systems. As shown by Martin (2006), outflows continue to larger radii in long slit spectra. Using larger radii 
in our SRFW model would only increase the measured masses, momenta, and energies.

The location of the neutral gas in nearby galaxies is not fully constrained because it is seen in absorption. However, absorption-line surveys can measure the average integrated covering factor of the neutral gas $(\Omega / 4 \pi ; \S 3.4 .1)$ by observing many different systems. As seen through nuclear lines of sight, the integrated covering factor increases with increasing star formation rate. Systems with star formation rate $(\mathrm{SFR}) \sim 1 M_{\odot} \mathrm{yr}^{-1}$ show $\Omega / 4 \pi \sim 0.1$ (Chen et al. 2010; Bouché et al. 2012), nearby LIRGs $\left(\mathrm{SFR}=7-70 M_{\odot} \mathrm{yr}^{-1}\right.$ ) have $\Omega / 4 \pi \sim 0.4$ (Rupke et al. $2005 \mathrm{c})$, and nearby ULIRGs (SFR $>70 M_{\odot} \mathrm{yr}^{-1}$ ) have $\Omega / 4 \pi \sim 0.8$ (Rupke et al. 2002, 2005c; Martin 2005). (In the model of a bipolar wind, these correspond to opening angles on one side of the disk of $60^{\circ}, 110^{\circ}$, and $160^{\circ}$, respectively.)

The integrated covering factors of the ionized and neutral gas can also be estimated from the SRFW, using the radii we assume $(\S 3.4 .1$. This way of estimating $\Omega / 4 \pi$ is much less certain, especially since we assume a wind radius. A typical value is $\Omega / 4 \pi \sim 0.1$, if we double the near-side covering factor to account for a far-side outflow (Table 4).

For the neutral gas, values of $\Omega / 4 \pi$ determined from the SRFW deprojection (0.1) are much lower than those based on observations of many nuclear sightlines (0.8). The explanation we favor for this discrepancy is the fact that the background continuum source declines in brightness with increasing projected radius. Thus, the neutral gas becomes increasingly difficult to detect with increasing projected radius. Furthermore, these larger radius sightlines dominate the measured $\Omega / 4 \pi$, once deprojected. Thus, the integrated covering factors measured from our IFS observations are lower limits to the true values, which are probably closer to $\sim 0.8$.

\subsection{Ionized Gas Excitation}

The excitation of the ionized gas in a wind serves as a signpost of galaxy-scale processes. In disk galaxies in the local universe, gas excitation reveals wind-driven shocks and ionization by the wind power source. Outflowing warm ionized gas is typically shock-excited in cases where the wind is driven by a starburst alone (Veilleux \& Rupke 2002 Sharp \& Bland-Hawthorn 2010; Rich et al. 2010), and photoionized by the AGN radiation field in cases where an AGN is also present (Veilleux et al. 2003, Sharp \& Bland-Hawthorn 2010).

In local mergers, shock ionization is also prevalent (Monreal-Ibero et al. 2006, 2010, Rich et al. 2011, Soto et al. 2012; Westmoquette et al. 2012). Within a given system, forbidden-to-recombination line ratios typically increase with increasing radius and decreasing $\mathrm{H} \alpha$ surface brightness (Monreal-Ibero et al. 2010; Westmoquette et al. 2012). This is suggestive of a line flux mixing model, with a varying contribution of star formation and shocks determining the line ratios (Rich et al. 2011, Westmoquette et al. 2012).

Merger shocks could be driven by tidally-induced motions or gas outflows. Monreal-Ibero et al. (2006, 2010) found a correlation between line ratios and gas velocity dispersion and argued for a tidal origin of merger shocks. High spectral resolution, multiple profile emis- sion line fitting, and comparison to shock models confirmed that more shock-like line ratios were associated with higher velocity dispersion (Rich et al. 2011). Soto et al. (2012) identified two subclasses of shock- (or possibly AGN-excited) gas - a subset with lower velocity dispersions $\left(\sigma \lesssim 150 \mathrm{~km} \mathrm{~s}^{-1}\right)$ which is consistent with a tidal origin and a subset with higher velocity dispersions $\left(\sigma \gtrsim 150 \mathrm{~km} \mathrm{~s}^{-1}\right)$ that is probably outflowing.

From our data, the $[\mathrm{N}$ II $] / \mathrm{H} \alpha$ and $[\mathrm{O} \mathrm{I}] / \mathrm{H} \alpha$ line ratio maps for the galaxies in our sample (e.g., Figure 14 are consistent with these previously determined relationships. In individual objects, line ratios in the rotating gas component anticorrelate with $\mathrm{H} \alpha$ flux, correlate with FWHM, and increase with increasing projected galactocentric radius. Within each system, small regions can deviate from these trends; careful inspection of the maps of each system show that the overall correlations do not tell the entire story. The exact nature of the spatial variation of gas excitation in each source is outside the scope of this work, particularly since we probe a limited number of strong emission lines.

However, we are able to improve on the analysis of Soto et al. (2012) by studying individual systems, and on the analysis of Rich et al. (2011) by being able to identify outflows. Figure 49 shows the ionized gas velocity (for outflowing components) as a function of FWHM, color-coded by $[\mathrm{N} \mathrm{II}] / \mathrm{H} \alpha$ emission-line ratio. In a simple mixing model between stellar photoionization and shock excitation and/or AGN photoionization, increasing the percentage of line flux from shock excitation or AGN photoionization to a particular spaxel will increase $[\mathrm{N} \mathrm{II}] / \mathrm{H} \alpha$.

We find that, within a given system, spaxels with a given FWHM show increased line ratios with increasing velocity $\left(v_{50 \%}\right)$ with respect to systemic. Correspondingly, spaxels with a given velocity show increased line ratios as FWHM increases. It is clear that bulk motions (as traced by $v_{50 \%}$ ) and unresolved bulk or randomized motions (as traced by FWHM) are both responsible for shock ionization of the broad emission line components in ULIRGs. In some systems (F10565+2448 and Mrk 273) these motions are due almost completely to powerful outflows. In others (VV 705:NW), some of the motions $\left(v_{50 \%} \lesssim-50 \mathrm{~km} \mathrm{~s}^{-1}\right.$ and FWHM $\left.\gtrsim 400 \mathrm{~km} \mathrm{~s}^{-1}\right)$ are clearly due to outflows and some $\left(\left|v_{50 \%}\right| \lesssim 50 \mathrm{~km} \mathrm{~s}^{-1}\right.$ and FWHM $\lesssim 400 \mathrm{~km} \mathrm{~s}^{-1}$ ) may be tidally induced motions.

In the three galaxies in our sample with luminous AGN, photoionization by the AGN could in principle contribute to the observed line ratios. In F08572+3915:NW, the SDSS spectrum shows no evidence of NLR emission, though the AGN in this source is heavily obscured. In Mrk 231, there is presently no published spatial information on extended high-ionization emission lines. Finally, in Mrk 273, there is AGNphotoionized gas with low velocities near the SW nucleus (Colina et al. 1999). However, the contribution of the AGN to the excitation of the outflowing gas in Mrk 273 is unclear (Colina et al. 1999). Future IFS observations of these sources in high-ionization lines will help to clarify the origin of ionization.

\subsection{The Multiphase Wind}


The present dataset permits the first spatially-resolved comparison between the neutral and ionized gas phases in merger winds. Previous spatially-resolved surveys have focused on either the ionized gas (Heckman et al. 1990, Lehnert \& Heckman 1996; Veilleux et al.| 2003; Sharp \& Bland-Hawthorn 2010; Westmoquette et al. 2012; Soto \& Martin 2012; Soto et al. 2012) or the neutral gas (Martin 2006). Single aperture surveys have produced evidence that the velocities of the neutral and ionized gas phases in merger winds are not identical or correlated on a system-to-system basis, except perhaps in the case of AGN (Heckman et al. 2000, Rupke et al. 2005a c). Soto et al. (2012) argue that the ionized gas in ULIRG winds is of comparable mass to the neutral gas.

In Figures 50 and 51, we show that there is a significant correlation between the (projected) neutral and ionized gas velocities within a given galaxy for three of the five galaxies in our sample with both ionized and neutral outflows: F10565+2448, Mrk 273, and VV 705:NW. This correlation is present whether $v_{50 \%}, v_{98 \%}$, or FWHM is considered. Furthermore, in all of the systems with both ionized and neutral outflows, the phase with higher velocity varies from point to point and object to object. In $2 / 5$ systems with both ionized and neutral outflows, the ionized gas is typically higher in velocity; in $2 / 5$ the situation is reversed; and in $1 / 5$ the velocities are comparable, on average.

The correlations we observe point to a physical connection between the ionized and neutral outflow phases within a given galaxy, even if this connection is complex. This complexity relates to unresolved substructure along the line of sight that may be different for each phase, as well as the fact that the tracers of these phases reflect the underlying gas properties in different ways (e.g., density: emission lines scale as $n^{2}$ and optically thin absorption lines as $n$ ).

Two systems, F08572+3915:NW and Mrk 273, show the highest velocities in our survey, $2000-3000 \mathrm{~km} \mathrm{~s}^{-1}$. Velocities this high are seen only in the ionized gas, and not the neutral gas (or molecular gas; see below). These high velocities are also only seen in galaxies with an AGN, a point to which we will return in the next section.

The ratio between the mass, momentum, and energy contained within the ionized and neutral phases varies from object to object (Table 7), but in all systems the neutral gas dominates the mass. In two systems (F08572+3915:NW and Mrk 273) the ionized gas contributes significantly to the momentum and energy. The ionized gas, however, is clearly affected by extinction in these systems. Correcting for extinction would only increase the contribution of the ionized gas to a system's mass, momentum, and energy. It is unclear how extinction corrections would affect the typical velocity of the gas. IFS observations of other Balmer lines in these systems (in order to correct for extinction), or of ionized gas in the NIR and MIR, would provide better constraints on the ionized gas properties of the wind.

The wind in F10565+2448 is also dusty, as shown by the correlation between continuum color and $\mathrm{Na}$ I D equivalent width on a spaxel-by-spaxel basis (Shih \& Rupke 2010 Figure 52 and the dust filaments emerging along the minor axis $(\S 4.2 .2$ and Figure 17$)$. Both lower covering factor and lower optical depth reduce the absorption line equivalent width. If the gas and dust are closely associated, then a lower covering factor makes the continuum bluer by allowing more light to emerge dustfree, and a lower optical depth makes it bluer by simply reddening the continuum to a lesser degree. However, the underlying stellar population can also vary with position, and will introduce scatter and/or slope changes (within a given system) or systematic offsets (on a system-tosystem basis).

The other galaxies in our dataset do not show the large dynamic range in $H S T$ F435W/F814W color and $\mathrm{Na}$ I D equivalent width that are seen in F10565+2448. F17207-0014 comes the closest, and shows a less significant correlation, with more scatter. The data for the other galaxies in our sample (except for Mrk 231, whose continuum structure is too strongly impacted by the central source for accurate colors) do not show correlations, but do lie on or near the correlation defined by $\mathrm{F} 10565+2448$.

If the neutral phase of these winds is indeed dusty, then this outflowing dust is partly responsible for obscuring the visible galaxy continuum. Most of the UV light in ULIRGs is heavily obscured by concentrations of nuclear dust (Soifer et al. 2000, Goldader et al. 2002); however, on larger scales outflowing dust may play an important role as a foreground screen in extinguishing the visible continuum. This effect is seen at high spatial resolution in the kpc-scale dust filaments in F10565+2448 (Figure 17). Future detailed analyses of the optical colors and other extinction probes (e.g., the Balmer decrement) will better illuminate this connection in other systems.

The winds in ULIRGs also have a molecular component (Fischer et al. 2010a, Feruglio et al. 2010, Sturm et al. 2011; Aalto et al. 2012; Cicone et al. 2012, Veilleux et al. 2013, in prep.). Except for the case of Mrk 231, the only information at present on the molecular phase in these systems comes from single-aperture Herschel observations of $\mathrm{OH}$ transitions. Three galaxies in our sample (F08572+3915:NW, Mrk 231, and F17207-0014) are in the 6-galaxy sample of Sturm et al. (2011). The single-aperture integrated velocity profiles are not directly comparable to our spatially-resolved data. However, it is clear that the high neutral gas velocities in F08572+3915:NW and Mrk 231 are also observed in the molecular gas. Sturm et al. (2011) measure $v_{50 \%}=-700$ and $-600 \mathrm{~km} \mathrm{~s}^{-1}$ and $v_{98 \%}=-1300$ and $-1200 \mathrm{~km} \mathrm{~s}^{-1}$, respectively, in these galaxies. These $v_{50 \%}\left(v_{98 \%}\right)$ values lie somewhere in between the average and maximum $v_{50 \%}\left(v_{98 \%}\right)$ values that we measure in the neutral gas for these two galaxies, suggesting that the neutral and molecular gas are connected. However, the ionized gas in F08572+3915 reaches much higher velocities. In F17207-0014 $\left(v_{50 \%}=-100\right.$ and maximum velocity of $-370 \mathrm{~km} \mathrm{~s}^{-1}$ in the molecular gas), the neutral gas shows significantly higher velocities than the molecular gas, though the highest velocity neutral gas is at large radius.

The presence of molecular outflows alongside minoraxis neutral and ionized flows in our IFS sample suggests a helpful picture. On kpc scales, the dusty, molecular, neutral, and ionized wind emerges along the minor axis of a molecular disk. The molecular gas is entrained as 
the wind emerges from this disk, and is roughly co-spatial with the neutral and ionized gas at small scales.

\subsection{Wind Power Sources}

Perhaps the most important outstanding issue in our understanding of ULIRG winds is the relative importance of a starburst and AGN in accelerating the wind in a particular galaxy. This is an important question because feedback from AGN and/or stars are frequently invoked in models to explain the emergence of QSOs from dusty, major mergers of disk galaxies (Sanders et al. 1988 ) and the subsequent truncation of star formation and black hole activity (Springel et al. 2005, Hopkins et al. 2005). Attention has recently turned to AGN feedback in particular in this context, due to its higher expected velocities compared to stellar feedback and its rapid emergence after the onset of AGN activity.

Indirect arguments for the presence of QSO-mode feedback in mergers abound, but until recently data have been slim. There is now evidence from multiple gas probes that the large-scale outflow in Mrk 231 is an example of such feedback (Rupke et al. 2005a) Fischer et al. 2010a, Feruglio et al. 2010; Sturm et al. 2011; Rupke \& Veilleux 2011; Aalto et al. 2012, Cicone et al. 2012). The argument for this outflow being AGN- rather than starburst-driven relies on the relative power of the central engine compared to the starburst, and on the remarkably high velocities observed, which are higher than those seen in ULIRGs with lower luminosity AGN or no AGN (Sturm et al. 2011; Rupke \& Veilleux 2011).

Surveys of outflows in mergers and other systems based on nuclear spectra suggested that starbursts are sufficient to power large-scale outflows in systems where both are present, and found that there was no strong evidence for the AGN dominating large-scale outflow dynamics (Rupke et al. 2005a; Krug et al. 2010). However, spatially-resolved and multiphase data, as in the case of Mrk 231, and the advent of precise nuclear AGN luminosities for individual major mergers (Veilleux et al. 2009) have the potential to alter this conclusion. Nuclear Herschel spectra of nearby ULIRGs (including Mrk 231) show a correlation of molecular outflow velocity with AGN luminosity (Sturm et al. 2011; Veilleux et al. 2013, in prep.). This suggests that these (unresolved) molecular outflows are powered primarily by an AGN at the highest velocities.

The current sample contains three systems whose infrared luminosity is clearly dominated by star formation, and for which there is no clear evidence of AGN activity: F10565+2448, VV 705:NW, and F17207-0014. Our sample also has three systems that contain luminous AGN: F08572+3915, Mrk 231, and Mrk 273. How do the outflows in the systems with AGN compare to those without?

\subsubsection{Comparison of Wind Structures}

Structurally, the winds in galaxies with and without AGN are not obviously different. The data are consistent with the presence of flows along the minor axis of a nuclear disk in almost every system, with or without an AGN. Furthermore, superbubbles (F08572+3915:NW and VV 705:NW) and widespread extended outflows (F10565+2448, Mrk 231, Mrk 273, and F17207-0014) are each present in nuclei with and without AGN. Ionized gas excitation correlates with $v_{50 \%}$ and FWHM in both types of systems, suggesting shock excitation (though AGN photoionization may still be present; $\S 5.2$.

A wind perpendicular to a nuclear disk is consistent with the structures of starburst-driven winds in the local universe. However, it differs from those of the narrow line region (NLR) outflows in nearby Seyfert galaxies, which have random orientations with respect to the galaxy disk Crenshaw \& Kraemer 2000, Crenshaw et al. 2000, Fischer et al. 2010b, 2011; Müller-Sánchez et al. 2011). A collimated outflow perpendicular to a galaxy disk is thus, at face value, more consistent with a starburst-driven wind than a canonical Seyfert NLR outflow. However, this inference is based on the study of local disk galaxies, rather than mergers with massive molecular disks and luminous AGN. The AGN in our sample, with luminosities $3-10 \times 10^{45} \mathrm{erg} \mathrm{s}^{-1}$ (Veilleux et al. 2009), are much brighter than Seyferts. They are in fact radio-quiet QSOs, whose narrow line region sizes can reach several kpc or more (Bennert et al. 2002; Greene et al. 2011). (NLR outflows in Seyferts are typically confined to subkpc scales.) The structure of NLR outflows in radio-quiet QSOs is not well understood. From our data, we cannot determine the exact relationship between the outflowing ionized gas in our sample and the classical NLR, since we do not observe high-ionization emission lines and previous data is inconclusive $(\S 5.2$.

It is entirely plausible that the nuclear molecular disk could, on sub-kpc scales, collimate and redirect an outflow driven by the AGN (see Veilleux et al. 2005, and references therein). Examples in the local universe include NGC 3079 (Cecil et al. 2001, 2002) and NGC 4388 (Veilleux et al. 2003). In fact, the current data point to exactly this occuring in the AGN in our sample, if these outflows are in fact AGN-driven. NIR IFS with adaptive optics would be able to probe the physics of launching and collimation at smaller scales.

\subsubsection{Comparison of Mass, Momentum, and Energy}

We computed the mass, momentum, and energy in the outflows in our sample using the SRFW and BSB models ( $\$ 3.4$ and Table 6). The mass and momentum in the wind do not differ significantly between galaxies with and without AGN. However, the energy outflow rates in the galaxies with AGN are a factor $\sim 4$ higher than those with no AGN, primarily because of higher velocities (\$5.4.3).

The fraction of the momentum and energy in the ionized phase (compared to the neutral phase) rises to over half in two of the AGN, and is negligible in the rest of our sample $(\S 5.3$ and Table 7). Again, this is due to the high velocities reached in the ionized gas components of these two systems.

Table 8 shows the energy outflow rate in the wind compared to the mechanical luminosity of the starburst in each system. The mechanical luminosity follows from the continuous starburst models of Leitherer et al. (1999), with a correction applied for a Salpeter IMF with lower mass cutoff of $0.1 M_{\odot}$. In the model of starburst winds driven by mechanical energy from stellar winds and supernovae, there is sufficient mechanical energy from the starburst in each system to power the outflow. For the galaxies without AGN, the efficiency with which this mechanical energy must be converted into the kinetic energy 
of the outflow is low: $3 \%$ to $13 \%$. However, for the AGNs in our sample, it is much higher: $100 \%$ for F $08572+3915$ and Mrk 231, and $\gtrsim 20 \%$ for Mrk 273 (with only the ionized phase accounted for in the latter case). A high thermalization efficiency is inferred for M82 (Strickland \& Heckman 2009), though much of the kinetic energy in this system may reside in the hot, X-ray emitting wind fluid rather than the ionized and neutral gas components. The fraction of the starburst mechanical energy that goes into the neutral and ionized phases is certainly less than the total thermalization efficiency, and quite possibly much less (Strickland \& Stevens 2000). The starburst thermalization efticiencies required for the winds in the AGN in our sample are thus uncomfortably high.

If instead the wind is driven by radiation pressure from the high luminosity in these systems (e.g., King 2003, Murray et al. 2005), then we can compare the available momentum injection rate $\left(\tau L_{\mathrm{IR}} / c\right.$; Murray et al. 2005) to the wind's momentum outflow rate. Table 8 and Figure 53 show that these numbers are comparable - i.e., $\tau$ must be near unity for these outflows to be driven by radiation pressure. Given that most of the luminosity of these systems emerges in the far-infrared, almost every photon emerging from the nuclear power sources interacts at least once with surrounding interstellar dusty gas. Thus, radiative driving with $\tau=1$ is a natural, though certainly not the only, explanation for the powerful outflows in these systems. The luminosity is injected at very different scales for the starburst and AGN, however, since the MIR sizes of the AGN in our sample are much smaller than the systems without AGN (Soifer et al. 2000; Imanishi et al. 2011).

Any radiation pressure on the wind results from both a starburst and any AGN. Thus, in cases where both are present, it is important to understand how much each is contributing to the outflow. Figure 53 shows that simply intercepting the radiation from the starburst in these systems requires the wind to be quite optically thick $(\tau \gtrsim 4)$ in at least two cases. In the radiation pressure model, it is simpler to assume that the entire luminosity of the system, from both starburst and AGN, are powering the outflow.

If an AGN drives the highest velocity flows in our sample, then the energy outflow rate in the wind is a small fraction of the AGN's luminosity $(0.1-0.4 \%)$, comfortably within the requirements of recent models of AGN feedback (Hopkins \& Elvis 2010; Faucher-Giguère et al. 2012). Table 8 shows the ratio of the energy outflow rate in the wind to the AGN luminosity in each system in our sample.

In summary, we reach several conclusions by studying the mass, momentum, and energy in these winds. (1) The outflow mass in our sample is independent of the galaxy's power source, within the errors. (2) The starbursts in our sample are consistent with driving outflows via radiation pressure or mechanical energy from stellar winds and supernovae. (3) The data are consistent with AGN playing a significant role in driving the outflow in those systems where an AGN is present (though the mass, momenta, and energy do not conclusively show that an AGN is contributing). The outflows in the AGN in our sample are overall more energetic, have a more energetic ionized phase, and require uncomfortably large thermalization efficiencies if they are driven solely by me- chanical energy from stellar winds and supernovae. In terms of momentum and energy, however, they are consistent with radiative driving by both the starburst and AGN or with recent models of AGN feedback.

\subsubsection{Comparison of Velocities}

The energetic winds in the AGN in our sample are largely a result of their large velocities compared to the starbursts. Table 3 shows the spatially-averaged and maximum values of $v_{50 \%}$ and $v_{98 \%}$ for each gas phase and each galaxy, and Figure 54 shows how these values depend on the AGN luminosity. We show the AGN luminosity computed from the average AGN fraction of the bolometric luminosity from six MIR diagnostics, as well as plausible uncertainty in this value using the range of luminosities computed from individual MIR diagnostics (Veilleux et al. 2009). We assume that the AGN luminosities are upper limits for the galaxies in our sample without evidence for AGN at other wavelengths.

Both galaxies with and without an AGN host fast outflows. In systems without an AGN, we find spatiallyaveraged $v_{50 \%}\left(v_{98 \%}\right)$ values of $-250(-450$ to -650$)$ $\mathrm{km} \mathrm{s}^{-1}$ in the neutral gas and $-100(-500) \mathrm{km} \mathrm{s}^{-1}$ in the ionized gas. The highest velocities (maximum $v_{98 \%}$ ) in each system (considering all spaxels and phases) range from $-900 \mathrm{~km} \mathrm{~s}^{-1}$ to $-1250 \mathrm{~km} \mathrm{~s}^{-1}$.

In systems with an AGN, we find spatially-averaged $v_{50 \%}\left(v_{98 \%}\right)$ values are -100 to $-400(-450$ to -900$)$ $\mathrm{km} \mathrm{s}^{-1}$ in the neutral gas and -200 to $-1500(-700$ to $-2800) \mathrm{km} \mathrm{s}^{-1}$ in the ionized gas. The highest velocities (maximum $v_{98 \%}$ ) reached in any spaxel in each system range from $-1450 \mathrm{~km} \mathrm{~s}^{-1}$ to $-3350 \mathrm{~km} \mathrm{~s}^{-1}$.

We conclude from this analysis that the presence of a QSO in major mergers yields higher outflow velocities. The two systems with the highest neutral gas velocities (Mrk 231 and F08572+3915) contain QSOs, and the two systems with the highest ionized gas velocities (Mrk 273 and F08572+3915) also contain QSOs. Though there is clearly overlap between the velocity distributions of the galaxies with and without AGN, only those with an AGN reach outflow velocities exceeding $-1200 \mathrm{~km} \mathrm{~s}^{-1}$, and every system with an AGN exceeds this velocity in at least one gas phase. Furthermore, only the ionized gas phase reaches velocities of $\geq 2000 \mathrm{~km} \mathrm{~s}^{-1}$, and only in mergers hosting a QSO. The same trend of outflow velocity with AGN luminosity is seen in the molecular phase, again with velocities lower than $2000 \mathrm{~km} \mathrm{~s}^{-1}$ (Sturm et al. 2011, Veilleux et al. 2013, in prep.), providing independent confirmation of this result.

How does deprojection affect these conclusions? In Mrk 273 and VV 705:NW, we can deproject in some areas of our data using the BSB model. Figure 55 shows how this deprojection affects the derived average velocities. In VV 705:NW, the system with the highest observed velocities in a non-AGN, the velocities change very little because the superbubble is observed face-on. In Mrk 273, however, the superbubble is highly inclined, and the derived velocities double. Thus, in these two sources, at least, deprojection only strengthens the above conclusions.

This conclusion differs from that of Rupke et al. (2005a) and Krug et al. (2010), who argued that the 
AGN in LIRGs and ULIRGs did not accelerate the wind to higher velocities. However, these previous studies relied on single-aperture spectra and a single gas phase (neutral), as well as incomplete information about the AGN content of individual systems. It is clear that combining spatially-resolved, multiphase information, as in the current study, with more precise AGN diagnostics is key to fully understanding the power sources of winds in these galaxies.

The maximum velocities we observe in the neutral phase of ULIRG winds are also higher than previously reported (Heckman et al. 2000: Rupke et al. 2002, 2005a c Martin 2005 2006). It is clear from our spatially resolved data that this is because the highest velocities are often not found along the line of sight to the optical galaxy nucleus.

The total luminosity of the system does not appear to determine the wind velocities in our sample (Figure 56). The range of infrared luminosities in our sample spans only a factor of four, and the two highest velocity systems are in the middle of the luminosity range. This is consistent with previous results (Rupke et al. 2005c a).

The high velocities, masses, and energies we observe in ULIRGs with luminous AGN are comparable to the properties of outflows inferred to arise on $\mathrm{kpc}$ scales in other radio-quiet QSOs (Moe et al. 2009, Dunn et al. 2010 Borguet et al. 2013). The spatial constraints in other radio-quiet QSOs depend on detailed photoionization modeling and are thus not as direct as the measurements presented here. If they are correct, the similarity of these two results suggests that such QSO-driven flows are a general property of radio-quiet QSOs, whether found in mergers or otherwise. A more detailed census of outflows in radio-quiet QSOs of different types is clearly in order.

Outflow velocities up to $1500-2000 \mathrm{~km} \mathrm{~s}^{-1}$ are also seen in what appear to be purely starburst objects: socalled Lyman-break analogs, and compact starbursts at $z \sim 0.6$ (Tremonti et al. 2007; Heckman et al. 2011; Diamond-Stanic et al. 2012 though obscured AGN have not been ruled out in these systems). The highest outflow velocities from these starbursts apparently require very compact star formation (Overzier et al. 2009 Heckman et al. 2011, Diamond-Stanic et al. 2012). ULIRGs also contain compact star formation, with star formation rate surface densities of $100-1000 M_{\odot} \mathrm{yr}^{-1}$ (based on their infrared luminosities and molecular disk radii; Downes \& Solomon 1998), comparable to Lyman break analogs and the $z \sim 0.6$ compact starbursts (Overzier et al. 2009 Diamond-Stanic et al. 2012). However, the compact star formation in ULIRGs is heavily obscured and occurs amid massive reservoirs of dense gas, while the Lyman-break analogs and $z \sim 0.6$ compact starbursts are UV-luminous and relatively unobscured (Heckman et al. 2005. Overzier et al. 2009: Diamond-Stanic et al. 2012). Our data shows that an AGN is required in ULIRGs to achieve the highest velocities. We speculate that the higher velocities observed in UV-luminous compact starbursts compared to starburst ULIRGs result from the outflow not having to transfer energy and momentum into a dusty, dense, massive interstellar medium around the starburst.

\subsection{Feedback in Major Mergers}

An important outstanding question is the impact of these massive, high-velocity flows on their host galaxies and nuclear black holes. These galaxies retain a central molecular disk (Downes \& Solomon 1998), as well as rapid star formation and active galactic nuclei, so any negative feedback has yet to be felt.

In Table 8, we show that the mass outflow rate, while uncertain, is a significant fraction of the star formation rate. Thus, the outflow evacuates gas at a rate similar to the star formation rate as it propagates through the galaxy. The outflow mass will only increase if we include the molecular phase (Feruglio et al. 2010, Sturm et al. 2011). Furthermore, the outtlow is highly mass-loaded, meaning that most of its mass comes from gas it has entrained while moving through the galaxy. Table $8 \mathrm{com}-$ pares the mass outflow rate (measured) with the mass input from the starburst itself (from models; Leitherer et al. 1999). These numbers suggest that, on average, about $75 \%$ of the wind mass comes from entrained material, and perhaps more when other gas phases are included.

As we argued in $\S 5.1 .1$, a comparison of the dynamical timescales of the wind $(\lesssim 10 \mathrm{Myr})$ and merger $(\sim 100 \mathrm{Myr})$ suggests that the duty cycle of winds is high and that these outflows are relatively continuous. Similarly, the timescale for star formation to deplete the nuclear molecular gas reservoir in these systems $\left(4-6 \times 10^{9} M_{\odot}\right.$ for the four galaxies with $\mathrm{CO}$ interferometric data; Downes \& Solomon 1998) is of order $30-40$ Myr (the average star formation rate in our sample is $\left.143 \mathrm{M}_{\odot} \mathrm{yr}^{-1}\right)$. The timescale for the wind to deplete the nuclear gas reservoir is slightly larger: $\sim 100 \mathrm{Myr}$, based on the mass outflow rates we calculate here. Thus, the gas consumption, mass outflow, and merger dynamical timescales are all similar, and all three processes will be important in regulating star formation.

The overall picture of star formation self-regulation is a complex one, but it is clear that gas outflows do play a role in governing star formation. Molecular gas is part of the dusty, neutral wind we detect here, and these outflows will serve to end star formation in particular regions on a shorter timescale than would the exhaustion of the gas reservoir by star formation itself. This is certainly one means of negative feedback on star formation.

Feedback to AGN accretion, however, is difficult to probe on these scales. Observations at smaller scales, comparison to simulations, and population studies may all be necessary to better constrain whether the fast AGN outflows in these systems are true feedback on the AGN itself, and on what timescales.

\section{SUMMARY}

In this study, we have presented deep integral field spectroscopy of the optical emission and absorption lines in one luminous and five ultraluminous infrared galaxies at $z \leq 0.06$. Three of these galaxies have dustenshrouded QSOs, and three have no apparent AGN. By fitting the Na I D absorption line and multiple Gaussian components to strong emission lines, we perform a spatial and spectral decomposition of the data. We are able to separate rotating from outflowing or tidally-disturbed gas, and have detected neutral and ionized outflows on 
kpc scales in all six galaxies. This detailed data set has enabled us to study the multiphase structure and power sources of the winds in these systems.

We reach a number of major conclusions.

1. Where CO maps of the rotation of a nuclear disk are available ( 4 of the 6 galaxies in our sample), the narrow component of the ionized gas matches the rotation of the molecular gas in those regions where they are coincident $(\oint 5.1 .1)$. On larger scales, the narrow component of the lonized gas combines a continuation of the rotation of the nuclear disk with tidally-induced kinematics.

2. In 5 of 6 galaxies, the dynamics and structure of at least one gas phase at projected galactocentric radii of $1-2 \mathrm{kpc}$ are consistent with a bipolar outflow collimated by the nuclear molecular disk ( $\$$ 5.1.1). In two galaxies (Mrk 273 and VV 705:NW), this bipolar outflow takes the form of a bipolar superbubble that is detected on the basis of clear kinematic signatures (Figures 29 and 41). On larger scales, the winds are less collimated. The prevalence of minor axis flows on small scales, and their short timescales compared to the merger dynamical time, suggests they are a somewhat continuous phenomenon.

3. Consistent with the results of Martin (2006), the winds in our systems reach projected galactocentric radii of at least $2-5 \mathrm{kpc}$, with the detectable size of the wind limited in most cases by either the size of our FOV or sensitivity.

4. Consistent with the results of Sharp \& BlandHawthorn (2010), Rich et al. (2011), and Soto et al. (2012), we find that the excitation of the broad component of the ionized gas is due primarily to wind-driven shocks, since emission line ratios in this component increase with both velocity with respect to systemic and with line width (Figure 49. A contribution from AGN photoionization is also likely in those galaxies with a QSO.

5. Within several systems, we find strong correlations between the velocities of the ionized and neutral gas phases on a spaxel-by-spaxel basis (Figures 50 and 51). The velocities are not often identical, however, and it is not yet clear what determines the relative velocities of the two phases.

6. We calculate that most of the mass, momentum, and energy in the wind is contained in the neutral gas phase (Table 7). The exceptions are the galaxies with the highest ionized gas velocities, which divide the momentum and energy roughly evenly between the ionized and neutral phases. However, the contribution from the ionized phase may be underestimated because of our inability to make extinction corrections, and uncertainties in the neutral gas values remain because of uncertain ionization corrections.

7. Spatially-resolved comparisons between the neutral phase of the wind and the optical continuum (Figures 17 and 52 show that the outflows are dusty in at least some cases, and thus partly responsible for obscuring the optical continuum emission in these systems on kpc scales.

8. In those systems without an AGN, the properties of the wind are consistent with being driven by radiation pressure or supernova mechanical energy. In those systems with a QSO, however, the required thermalization efficiencies appear too high to explain the energetics on the basis of supernova mechanical energy alone. Furthermore, if radiation pressure from a starburst alone drove the outflow in the QSOs, the wind optical depth would need to exceed unity by a factor of $\sim 4$. In the systems with a QSO, the data are consistent with the wind being driven by a combination of radiation pressure and/or mechanical energy from the starburst and QSO $(\$ 5.4 .2$.

9. The galaxies with QSOs have the highest outflow velocities (Figure 54), reaching projected velocities of at least $1450 \mathrm{~km} \mathrm{~s}^{-1}$ in each system with a QSO, and up to $3350 \mathrm{~km} \mathrm{~s}^{-1}$ in one (F08572+3915:NW). Galaxies without AGN reach maximum projected velocities of $\sim 1000 \mathrm{~km} \mathrm{~s}^{-1}$. We conclude that the QSO plays an important role in accelerating the wind in those systems in which a QSO is present.

10. The highest gas velocities $\left(2000-3000 \mathrm{~km} \mathrm{~s}^{-1}\right)$ are reached in the ionized gas of two buried QSOs (Mrk 273 and F08572+3915). Gas velocities this high are not seen in the neutral gas.

11. When the ionized, neutral, and molecular phases are combined, these merger outflows are massive enough to evacuate star-forming gas from the galaxy at a rate comparable to the star formation rate, suggesting that negative feedback is occurring $(\S 5.5)$.

Besides these scientific conclusions, the results of our study also show that single aperture nuclear spectra, or even long slit spectra, do not adequately capture the structural complexity of winds in major mergers. In particular, IFS data is required to disentangle gas motions due to rotation, tidal motions, and outflow. Furthermore, peak velocities measured in nuclear apertures, or along slits in a random direction, are not likely to probe the peak velocities in the outflow, which in our data often occur outside the nuclear line of sight.

Though we are able to draw important conclusions from this sample, it is clear that its scope is limited by its size. Larger IFS samples of ULIRGs will put on firmer footing, or modify, the conclusions reached here. Observations at higher spatial resolution (with, e.g., NIR IFS adaptive optics observations) will further illuminate the launching region and mechanism of these winds, particularly in those systems where the wind is poorly resolved (the ionized phases in F08572+3915 and Mrk 231) or where there are multiple, closely-spaced nuclei (Mrk 273). In QSO-powered systems, studying the inner regions will also help to determine the physical mechanism by which the AGN helps to power the outflow. Finally, observations of other emission lines (e.g., $\mathrm{H} \beta$ and [O III]) will enable us to better constrain the 
connection of the dust to the ionized gas phase, the mass of the ionized gas phase, and the ionization mechanism of the emission line gas.

The authors are pleased to thank Alex Piazza for creating the HST color maps; Vivian U and Anne Medling for sharing their data and conclusions on Mrk 273 prior to publication; Nahum Arav, Mike Crenshaw, Aleks Diamond-Stanic, Claude-Andre Faucher-Giguere, Travis Fischer, Crystal Martin, Kurt Soto, and Christy Tremonti for helpful conversations; the organizers of the 2011 Ringberg, AGN Winds in Charleston, and NRAO Jets and Outflows meetings; and Jenny Shih for her work in prior stages of this project. We also thank David Fanning and Craig Markwardt for their excellent IDL software packages. Finally, we are grateful for the detailed comments of the anonymous referee, whose input significantly improved the paper. D. S. N. R. was supported by NASA through a Herschel data analysis award, by the Rhodes College Faculty Development Endowment, and by a Cottrell College Science Award.

This work was based primarily on observations obtained at the Gemini Observatory (program IDs GN2007A-Q-107 and GN-2010A-Q-41), which is operated by the Association of Universities for Research in Astronomy, Inc., under a cooperative agreement with the NSF on behalf of the Gemini partnership: the NSF (United States), the Science and Technology Facilities Council (United Kingdom), the National Research Council (Canada), CONICYT (Chile), the Australian Research Council (Australia), Ministério da Ciência, Tecnologia e Inovação (Brazil) and Ministerio de Ciencia, Tecnología e Innovación Productiva (Argentina).

The HST observations described herein were obtained from the Hubble Legacy Archive, which is a collaboration between the Space Telescope Science Institute (STScI/NASA), the Space Telescope European Coordinating Facility (ST-ECF/ESA) and the Canadian Astronomy Data Centre (CADC/NRC/CSA).

Small portions of this work are based on observations made with the Spitzer Space Telescope, obtained from the NASA/ IPAC Infrared Science Archive, both of which are operated by the Jet Propulsion Laboratory, California Institute of Technology under a contract with NASA.

Aspects of this work have made use of the SDSS. Funding for the SDSS and SDSS-II has been provided by the Alfred P. Sloan Foundation, the Participating Institutions, the NSF, the U.S. Department of Energy, NASA, the Japanese Monbukagakusho, the Max Planck Society, and the Higher Education Funding Council for England. The SDSS Web Site is http://www.sdss.org/

\section{REFERENCES}

Aalto, S., Garcia-Burillo, S., Muller, S., Winters, J. M., van der Werf, P., Henkel, C., Costagliola, F., \& Neri, R. 2012, A\&A, 537, A44

Adams, T. F., \& Weedman, D. W. 1972, ApJ, 173, L109

Allington-Smith, J., et al. 2002, PASP, 114, 892

Armus, L., Heckman, T. M., \& Miley, G. K. 1990, ApJ, 364, 471

Armus, L., et al. 2007, ApJ, 656, 148

Arribas, S., \& Colina, L. 2003, ApJ, 591, 791

Arribas, S., Colina, L., Monreal-Ibero, A., Alfonso, J.,

García-Marín, M., \& Alonso-Herrero, A. 2008, A\&A, 479, 687
Baan, W. A., \& Klöckner, H.-R. 2006, A\&A, 449, 559

Barnes, J. E., \& Hernquist, L. 1996, ApJ, 471, 115

Bennert, N., Falcke, H., Schulz, H., Wilson, A. S., \& Wills, B. J. 2002, ApJ, 574, L105

Bland, J., \& Tully, B. 1988, Nature, 334, 43

Boksenberg, A., Carswell, R. F., Allen, D. A., Fosbury, R. A. E. Penston, M. V., \& Sargent, W. L. W. 1977, MNRAS, 178, 451

Bondi, M., Pérez-Torres, M.-A., Dallacasa, D., \& Muxlow,

T. W. B. 2005, MNRAS, 361, 748

Borguet, B. C. J., Arav, N., Edmonds, D., Chamberlain, C., \& Benn, C. 2013, ApJ, 762, 49

Bouché, N., Hohensee, W., Vargas, R., Kacprzak, G. G., Martin, C. L., Cooke, J., \& Churchill, C. W. 2012, MNRAS, 3207

Bush, S. J., Wang, Z., Karovska, M., \& Fazio, G. G. 2008, ApJ, 688,875

Carilli, C. L., \& Taylor, G. B. 2000, ApJ, 532, L95

Cecil, G., Bland-Hawthorn, J., \& Veilleux, S. 2002, ApJ, 576, 745

Cecil, G., Bland-Hawthorn, J., Veilleux, S., \& Filippenko, A. V. 2001, ApJ, 555, 338

Chen, Y.-M., Tremonti, C. A., Heckman, T. M., Kauffmann, G., Weiner, B. J., Brinchmann, J., \& Wang, J. 2010, AJ, 140, 445

Chini, R., Kruegel, E., \& Steppe, H. 1992, A\&A, 255, 87

Cicone, C., Feruglio, C., Maiolino, R., Fiore, F., Piconcelli, E., Menci, N., Aussel, H., \& Sturm, E. 2012, A\&A, 543, A99

Cole, G. H. J., Pedlar, A., Holloway, A. J., \& Mundell, C. G. 1999, MNRAS, 310, 1033

Colina, L., Arribas, S., \& Borne, K. D. 1999, ApJ, 527, L13

Colina, L., Arribas, S., \& Monreal-Ibero, A. 2005, ApJ, 621, 725

Condon, J. J., Helou, G., Sanders, D. B., \& Soifer, B. T. 1990, ApJS, 73, 359

Condon, J. J., Huang, Z.-P., Yin, Q. F., \& Thuan, T. X. 1991, ApJ, 378, 65

Crenshaw, D. M., \& Kraemer, S. B. 2000, ApJ, 532, L101

Crenshaw, D. M., et al. 2000, AJ, 120, 1731

Dale, D. A., et al. 2000, AJ, 120, 583

Diamond-Stanic, A. M., Moustakas, J., Tremonti, C. A., Coil, A. L., Hickox, R. C., Robaina, A. R., Rudnick, G. H., \& Sell, P. H. 2012, ApJ, 755, L26

Downes, D., \& Solomon, P. M. 1998, ApJ, 507, 615

Dudik, R. P., Satyapal, S., \& Marcu, D. 2009, ApJ, 691, 1501

Dudley, C. C., \& Wynn-Williams, C. G. 1997, ApJ, 488, 720

Dunn, J. P., et al. 2010, ApJ, 709, 611

Evans, A. S., Mazzarella, J. M., Surace, J. A., \& Sanders, D. B. 2002, ApJ, 580, 749

Farrah, D., et al. 2007, ApJ, 667, 149

Faucher-Giguère, C.-A., Quataert, E., \& Murray, N. 2012, MNRAS, 420, 1347

Feruglio, C., Maiolino, R., Piconcelli, E., Menci, N., Aussel, H., Lamastra, A., \& Fiore, F. 2010, A\&A, 518, L155

Fischer, J., et al. 2010a, A\&A, 518, L41

Fischer, T. C., Crenshaw, D. M., Kraemer, S. B., Schmitt, H. R., Mushotsky, R. F., \& Dunn, J. P. 2011, ApJ, 727, 71

Fischer, T. C., Crenshaw, D. M., Kraemer, S. B., Schmitt, H. R., \& Trippe, M. L. 2010b, AJ, 140, 577

Franceschini, A., et al. 2003, MNRAS, 343, 1181

Genzel, R., et al. 1998, ApJ, 498, 579

Goldader, J. D., Meurer, G., Heckman, T. M., Seibert, M. Sanders, D. B., Calzetti, D., \& Steidel, C. C. 2002, ApJ, 568, 651

Greene, J. E., Zakamska, N. L., Ho, L. C., \& Barth, A. J. 2011, ApJ, 732, 9

Heckman, T. M., Armus, L., \& Miley, G. K. 1987, AJ, 93, 276

-. 1990, ApJS, 74, 833

Heckman, T. M., Lehnert, M. D., Strickland, D. K., \& Armus, L. 2000, ApJS, 129, 493

Heckman, T. M., et al. 2005, ApJ, 619, L35

-. 2011, ApJ, 730, 5

Hook, I. M., Jørgensen, I., Allington-Smith, J. R., Davies, R. L., Metcalfe, N., Murowinski, R. G., \& Crampton, D. 2004, PASP, 116,425

Hopkins, P. F., \& Elvis, M. 2010, MNRAS, 401, 7

Hopkins, P. F., Hernquist, L., Cox, T. J., Di Matteo, T., Martini, P., Robertson, B., \& Springel, V. 2005, ApJ, 630, 705

Imanishi, M., \& Dudley, C. C. 2000, ApJ, 545, 701

Imanishi, M., Imase, K., Oi, N., \& Ichikawa, K. 2011, AJ, 141, 156

Iwasawa, K., et al. 2011a, A\&A, 529, A106

-. 2011b, A\&A, 528, A137 
Kennicutt, R. C., J. 1998, ARA\&A, 36, 189

Kewley, L. J., Groves, B., Kauffmann, G., \& Heckman, T. 2006, MNRAS, 372, 961

Kim, D.-C., Sanders, D. B., Veilleux, S., Mazzarella, J. M., \& Soifer, B. T. 1995, ApJS, 98, 129

Kim, D.-C., Veilleux, S., \& Sanders, D. B. 1998, ApJ, 508, 627 King, A. 2003, ApJ, 596, L27

Knapen, J. H., Laine, S., Yates, J. A., Robinson, A., Richards, A. M. S., Doyon, R., \& Nadeau, D. 1997, ApJ, 490, L29

Koski, A. T. 1978, ApJ, 223, 56

Krug, H. B., Rupke, D. S. N., \& Veilleux, S. 2010, ApJ, 708, 1145

Lehnert, M. D., \& Heckman, T. M. 1996, ApJ, 462, 651

Leitherer, C., et al. 1999, ApJS, 123, 3

Majewski, S. R., Hereld, M., Koo, D. C., Illingworth, G. D., \& Heckman, T. M. 1993, ApJ, 402, 125

Martin, C. L. 1998, ApJ, 506, 222

-. 2005, ApJ, 621, 227

-. 2006, ApJ, 647, 222

Mihos, J. C., \& Hernquist, L. 1996, ApJ, 464, 641

Moe, M., Arav, N., Bautista, M. A., \& Korista, K. T. 2009, ApJ, 706,525

Momjian, E., Romney, J. D., Carilli, C. L., Troland, T. H., \& Taylor, G. B. 2003, ApJ, 587, 160

Monreal-Ibero, A., Arribas, S., \& Colina, L. 2006, ApJ, 637, 138

Monreal-Ibero, A., Arribas, S., Colina, L., Rodríguez-Zaurín, J., Alonso-Herrero, A., \& García-Marín, M. 2010, A\&A, 517, A28

Moustakas, J., \& Kennicutt, Jr., R. C. 2006, ApJS, 164, 81

Müller-Sánchez, F., Prieto, M. A., Hicks, E. K. S., Vives-Arias, H., Davies, R. I., Malkan, M., Tacconi, L. J., \& Genzel, R. 2011, ApJ, 739, 69

Murphy, Jr., T. W., Armus, L., Matthews, K., Soifer, B. T., Mazzarella, J. M., Shupe, D. L., Strauss, M. A., \& Neugebauer, G. 1996, AJ, 111, 1025

Murray, N., Quataert, E., \& Thompson, T. A. 2005, ApJ, 618, 569

Osterbrock, D. E. 1989, Astrophysics of gaseous nebulae and active galactic nuclei (Sausalito, CA, University Science Books, 1989)

Overzier, R. A., et al. 2009, ApJ, 706, 203

Prochaska, J. X., Kasen, D., \& Rubin, K. 2011, ApJ, 734, 24

Rich, J. A., Dopita, M. A., Kewley, L. J., \& Rupke, D. S. N. 2010, ApJ, 721, 505

Rich, J. A., Kewley, L. J., \& Dopita, M. A. 2011, ApJ, 734, 87

Rupke, D. S., Veilleux, S., \& Sanders, D. B. 2002, ApJ, 570, 588

-. 2005a, ApJ, 632, 751

-. 2005b, ApJS, 160, 87

-. 2005c, ApJS, 160, 115

Rupke, D. S. N., Kewley, L. J., \& Chien, L. 2010, ApJ, 723, 1255

Rupke, D. S. N., \& Veilleux, S. 2011, ApJ, 729, L27

Sanders, D. B., Mazzarella, J. M., Kim, D.-C., Surace, J. A., \& Soifer, B. T. 2003, AJ, 126, 1607

Sanders, D. B., \& Mirabel, I. F. 1996, ARA\&A, 34, 749

Sanders, D. B., Scoville, N. Z., \& Soifer, B. T. 1991, ApJ, 370, 158
Sanders, D. B., Soifer, B. T., Elias, J. H., Madore, B. F., Matthews, K., Neugebauer, G., \& Scoville, N. Z. 1988, ApJ, 325,74

Savage, B. D., \& Sembach, K. R. 1996, ARA\&A, 34, 279

Scoville, N. Z., et al. 2000, AJ, 119, 991

Sharp, R. G., \& Bland-Hawthorn, J. 2010, ApJ, 711, 818

Shih, H., \& Rupke, D. S. N. 2010, ApJ, 724, 1430

Smith, H. E., Lonsdale, C. J., \& Lonsdale, C. J. 1998, ApJ, 492, 137

Soifer, B. T., Neugebauer, G., Matthews, K., Egami, E., \&

Weinberger, A. J. 2002, AJ, 124, 2980

Soifer, B. T., et al. 2000, AJ, 119, 509

Soto, K. T., \& Martin, C. L. 2010, ApJ, 716, 332

—. 2012, ApJS, 203, 3

Soto, K. T., Martin, C. L., Prescott, M. K. M., \& Armus, L. 2012, ApJ, 757, 86

Springel, V., Di Matteo, T., \& Hernquist, L. 2005, MNRAS, 361, 776

Strickland, D. K., \& Heckman, T. M. 2009, ApJ, 697, 2030

Strickland, D. K., \& Stevens, I. R. 2000, MNRAS, 314, 511

Sturm, E., et al. 2011, ApJ, 733, L16

Surace, J. A., Sanders, D. B., \& Mazzarella, J. M. 2004, AJ, 127, 3235

Surace, J. A., Sanders, D. B., Vacca, W. D., Veilleux, S., \& Mazzarella, J. M. 1998, ApJ, 492, 116

Teng, S. H., \& Veilleux, S. 2010, ApJ, 725, 1848

Teng, S. H., et al. 2009, ApJ, 691, 261

Tremonti, C. A., Moustakas, J., \& Diamond-Stanic, A. M. 2007, ApJ, 663, L77

U, V., et al. 2012, ApJ, submitted

Veilleux, S., Cecil, G., \& Bland-Hawthorn, J. 2005, ARA\&A, 43, 769

Veilleux, S., Cecil, G., Bland-Hawthorn, J., Tully, R. B., Filippenko, A. V., \& Sargent, W. L. W. 1994, ApJ, 433, 48

Veilleux, S., Kim, D.-C., \& Sanders, D. B. 1999a, ApJ, 522, 113 —. 2002, ApJS, 143, 315

Veilleux, S., Kim, D.-C., Sanders, D. B., Mazzarella, J. M., \& Soifer, B. T. 1995, ApJS, 98, 171

Veilleux, S., \& Rupke, D. S. 2002, ApJ, 565, L63

Veilleux, S., Sanders, D. B., \& Kim, D.-C. 1999b, ApJ, 522, 139

Veilleux, S., Shopbell, P. L., Rupke, D. S., Bland-Hawthorn, J., \& Cecil, G. 2003, AJ, 126, 2185

Veilleux, S., et al. 2006, ApJ, 643, 707

一. 2009, ApJS, 182, 628

Westmoquette, M. S., Clements, D. L., Bendo, G. J., \& Khan, S. A. 2012, MNRAS, 3173

Wilson, C. D., et al. 2008, ApJS, 178, 189

Yao, L., Seaquist, E. R., Kuno, N., \& Dunne, L. 2003, ApJ, 588, 771

Yates, J. A., Richards, A. M. S., Wright, M. M., Collett, J. L.,

Gray, M. D., Field, D., \& Cohen, R. J. 2000, MNRAS, 317, 28

Yuan, T.-T., Kewley, L. J., \& Sanders, D. B. 2010, ApJ, 709, 884 
(a) F435W+F814W, 1’x1'

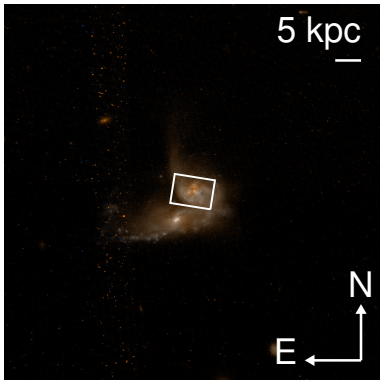

(e) $\log (\mathrm{F} 435 \mathrm{~W} / \mathrm{F} 814 \mathrm{~W})$

$1^{\prime} \times 1^{\prime}$

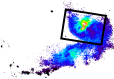

(b) F435W+F814W, 15"×15"

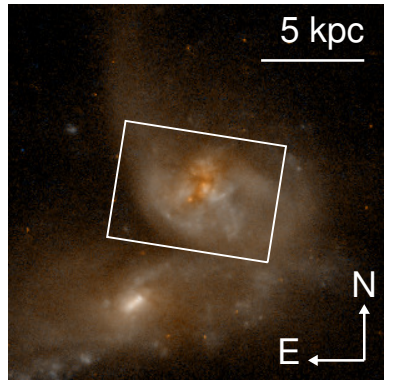

(f) $\log (F 435 W / F 814 W)$ $15 " \times 15 "$

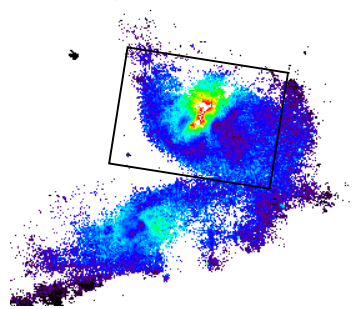

(c) 6100-6800

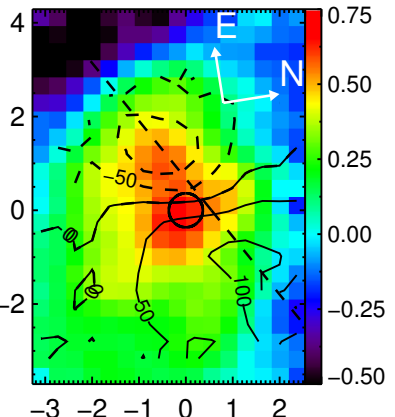

(g) $\log (F 435 W / F 814 W)$ GMOS FOV

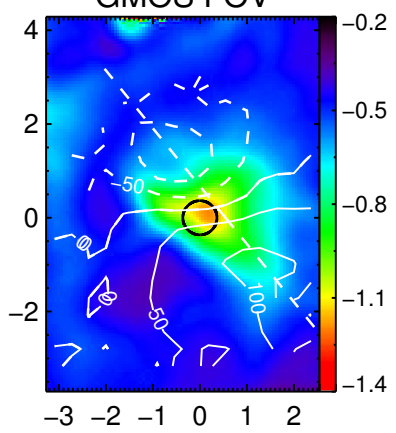

(d) $W_{\mathrm{Na} D}(\AA)$

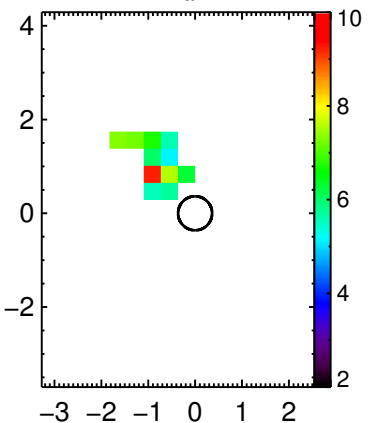

(h) $\mathrm{W}_{\mathrm{NaD}} / \delta \mathrm{W}_{\mathrm{NaD}}$

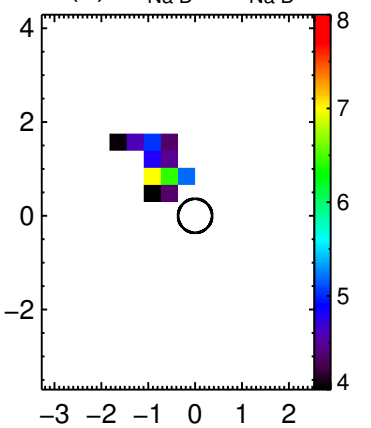

Figure 1. $(a)-(b)$ : Two-color $H S T$ images of F08572+3915:NW at sizes $1^{\prime} \times 1^{\prime}$ and $15^{\prime \prime} \times 15^{\prime \prime}$. Red is $F 814 W$, blue is $F 435 W$, and green is their average. The image orientation is indicated by the compass rose. The FOV of the GMOS observations is indicated by the box. (c) Logarithm of total GMOS flux integrated from 6100 to $6800 \AA$, in arbitrary units. The image orientation is indicated by the compass rose, and the axis labels are in units of kpc. The near-infrared (NIR) continuum peak is circled. Isovelocity contours of the narrow ionized gas component are overplotted and labeled with velocity in $\mathrm{km} \mathrm{s}^{-1}$. Velocities are relative to $z_{s y s}=0.0584$. The dashed line intersects the peaks in rotational velocity and has $\mathrm{PA}=120^{\circ} \mathrm{E}$ of $\mathrm{N}$. $(d)$ Rest-frame equivalent width of $\mathrm{Na} \mathrm{I} \mathrm{D}$, computed from line fits, in $\AA$. $(e)-(f)$ Maps of the HST continuum flux ratio, $\log (\mathrm{F} 435 \mathrm{~W} / \mathrm{F} 814 \mathrm{~W})$, with sizes and orientations identical to $(a)$ and $(b)$, respectively. $(g)$ Map of HST color within the GMOS FOV, rotated and smoothed with a Gaussian kernel of FWHM $=1^{\prime \prime}$. $(h)$ Signal-to-noise ratio of Na I D equivalent width. 
(a) F160W

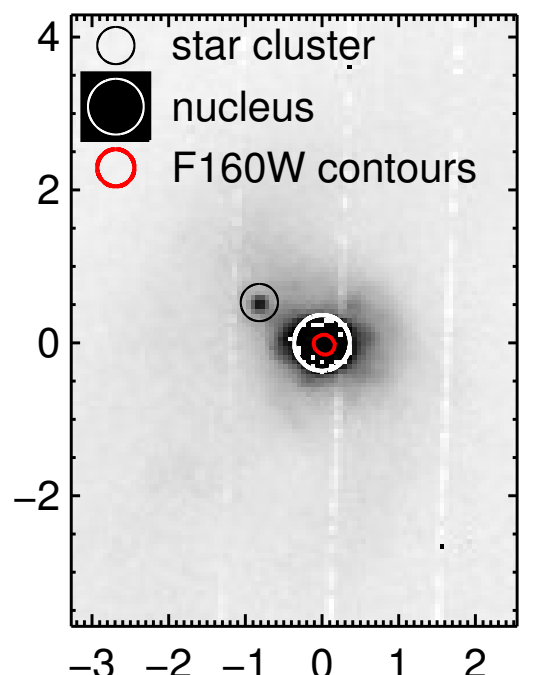

(c) F814W, smoothed

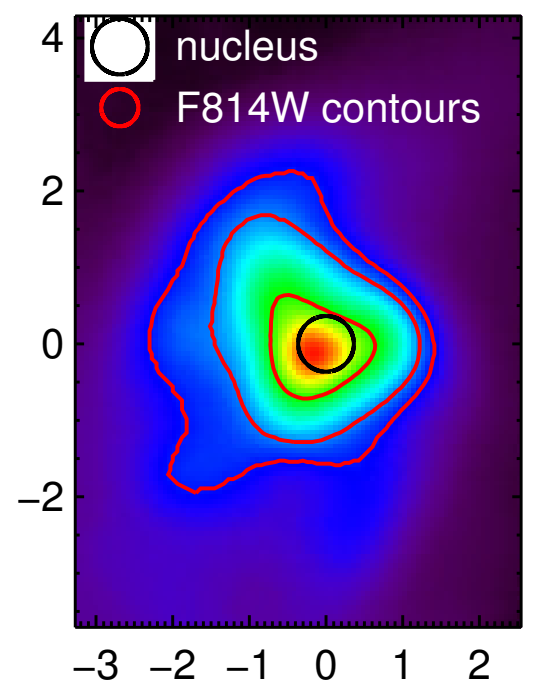

(b) F814W
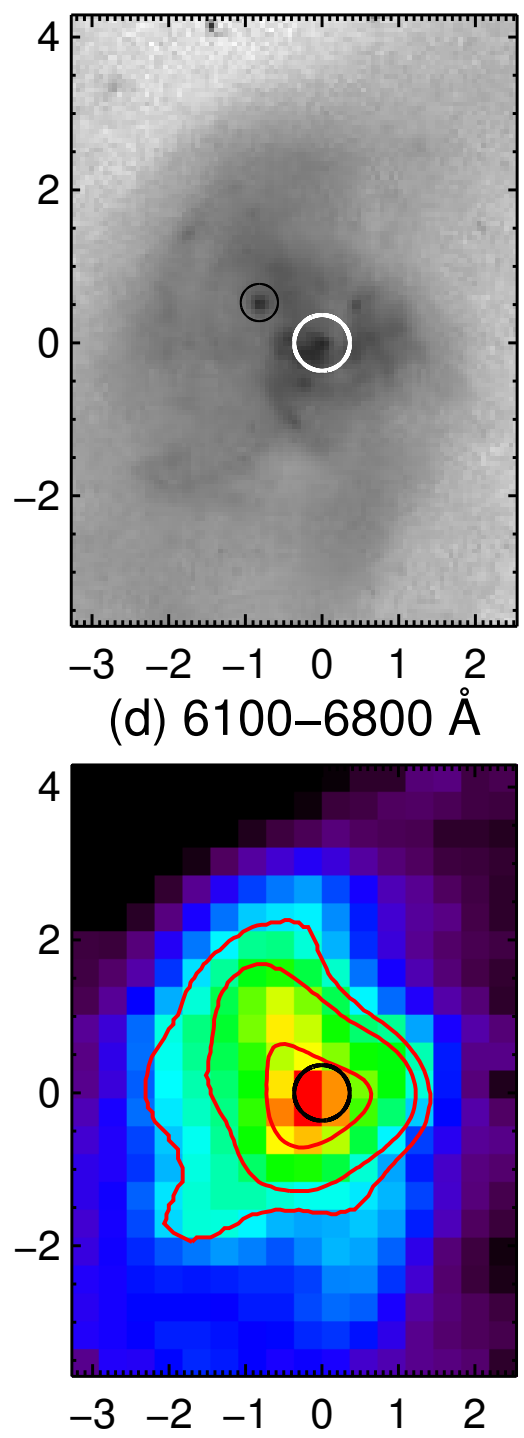

Figure 2. (a) HST F160W image of F08572+3915:NW, within the GMOS FOV. A contour highlighting the nucleus is in red. The nuclear star cluster used to align the images is circled, as is the NIR nucleus. Slight defects resulting from interpolation over detector artifacts appear near the nucleus but do not affect centering. (b) HST/ACS F814W image. Note the common alignment of the star cluster between this image and the F160W image, and the relative faintness of the nucleus in the optical. (c) F814W image, smoothed with a Gaussian kernel of $\sigma=00^{\prime \prime} 25$ to match ground-based seeing. Image contours are in red, and the NIR nucleus is circled. (d) GMOS $6100-6800 \AA$ image. Smoothed F814W contours are in red. Note that the smoothed F814W image is well aligned with the GMOS image. The location of the nucleus in the GMOS data is tied to the F160W image by common alignment with the F814W image. Axis labels are in kpc. 

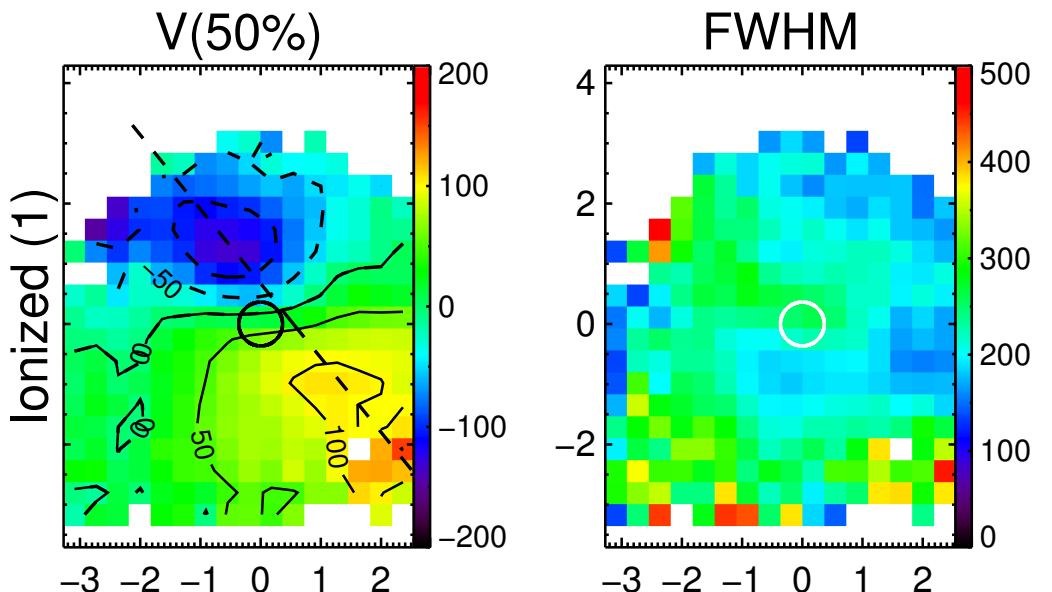

$\mathrm{V}(98 \%)$
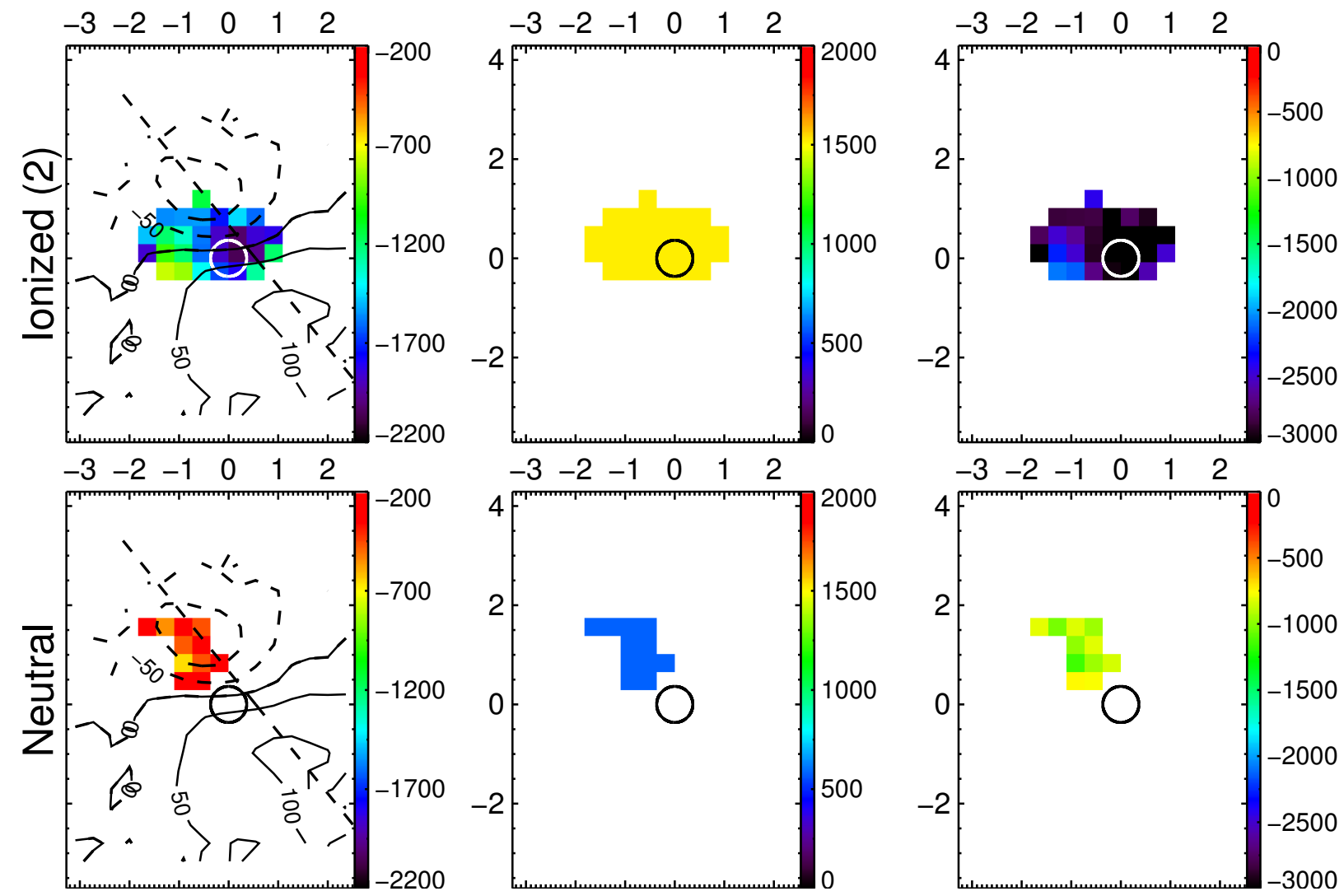

Figure 3. For F08572+3915: NW, maps of the centroid velocity $\left(v_{50 \%}\right)$, FWHM, and most blueshifted velocity $\left(v_{98} \% v_{50 \%}-2 \sigma\right)$ in each velocity component in the ionized gas (based on emission lines) and neutral gas (based on Na I D absorption). Each column shows a different velocity measure, and each row a different gas phase or velocity component. The ionized gas velocity component is labeled in parentheses $\left(1=\right.$ narrow, rotating component; 2 = broad, outflowing component). Colors indicate velocity in $\mathrm{km} \mathrm{s}^{-1}$. FWHM is corrected in quadrature for the instrumental profile. The contours, dashed lines, and open circles have the same meaning as in Figure 1 

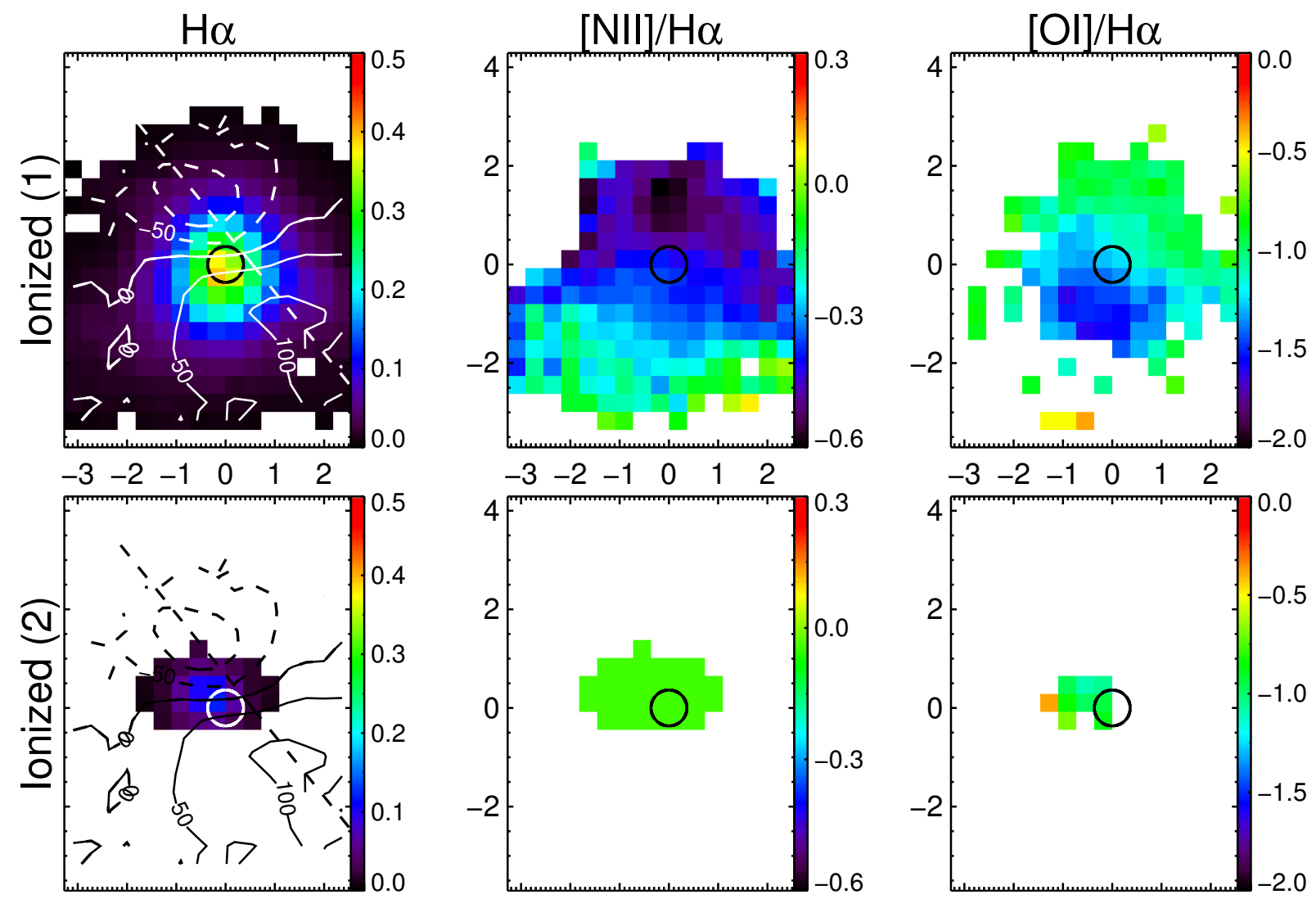

Figure 4. For F08572+3915:NW, maps of $\mathrm{H} \alpha$ flux in units of $1.0 \times 10^{-15} \mathrm{erg} \mathrm{s}^{-1} \mathrm{~cm}^{-2}$ per spaxel, or $1.1 \times 10^{-14} \mathrm{erg} \mathrm{s}^{-1} \mathrm{~cm}^{-2}$ $\operatorname{arcsec}^{-2}$ (left column), [N II] $6583 \AA / \mathrm{H} \alpha$ flux ratio (central column) and [O I] $6300 \AA / \mathrm{H} \alpha$ flux ratio (right column) in each ionized gas velocity component. The ionized gas velocity component is labeled in parentheses $(1=$ narrow, rotating component; $2=$ broad, outflowing component). The contours, dashed lines, and open circles have the same meaning as in Figure 1 . 


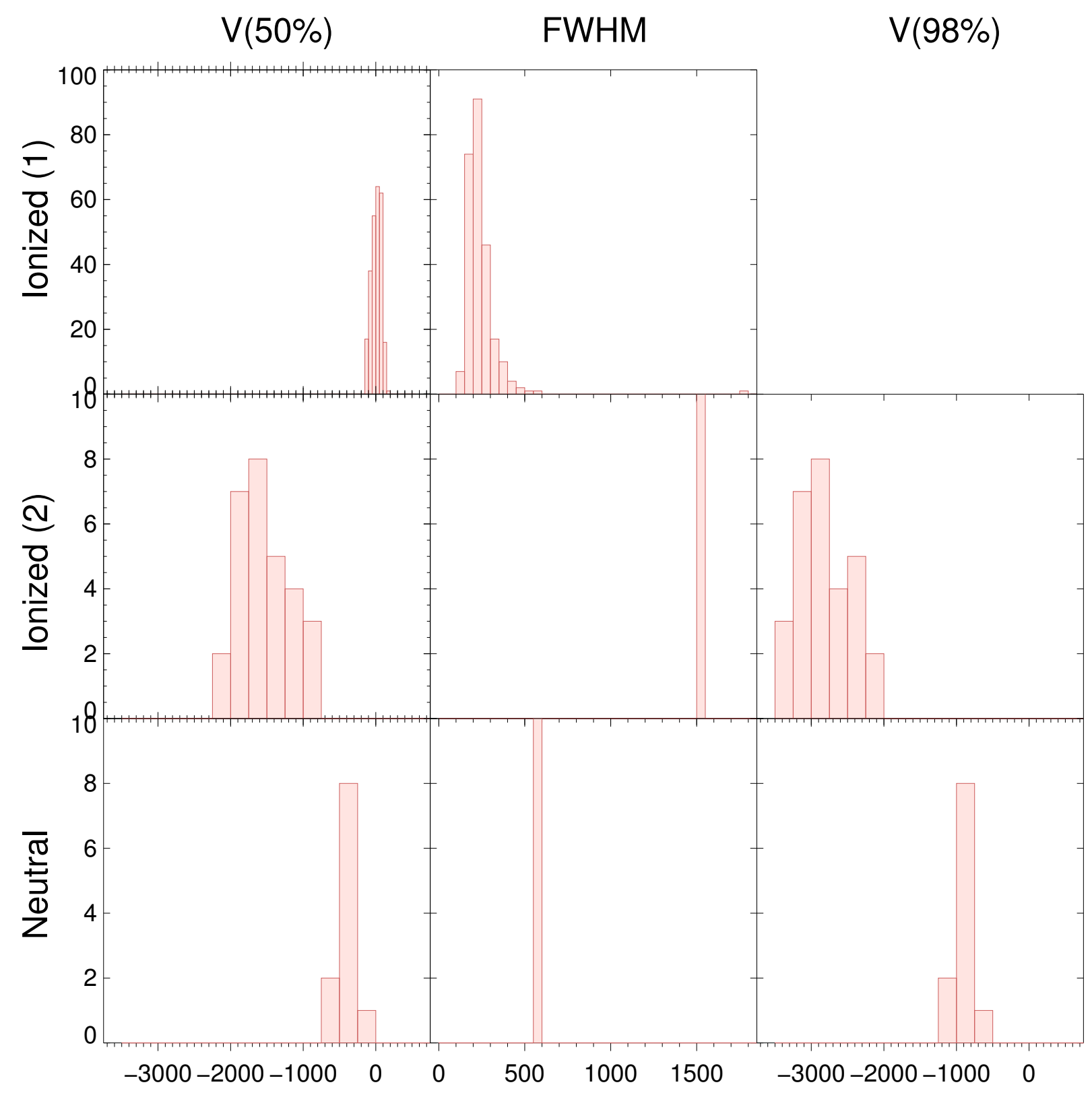

Figure 5. Distributions of $v_{50 \%}, \mathrm{FWHM}$, and $v_{98 \%}$ in each ionized gas component and the neutral gas in F08572+3915:NW. FWHM is corrected in quadrature for the instrumental profile. The vertical axis shows the number of spaxels in each bin, and the horizontal axis velocity in $\mathrm{km} \mathrm{s}^{-1}$. 
(a) F435W+F814W, 1'x1' (b) F435W+F814W, 15"×15"

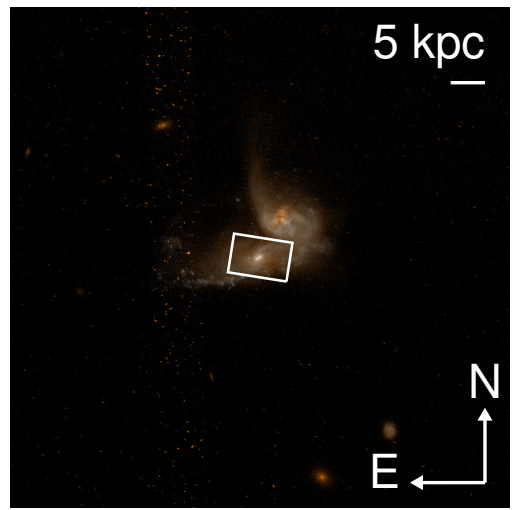

(d) $\log (\mathrm{F} 435 \mathrm{~W} / \mathrm{F} 814 \mathrm{~W})$ $1^{\prime} \times 1^{\prime}$

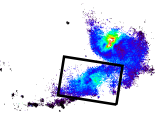

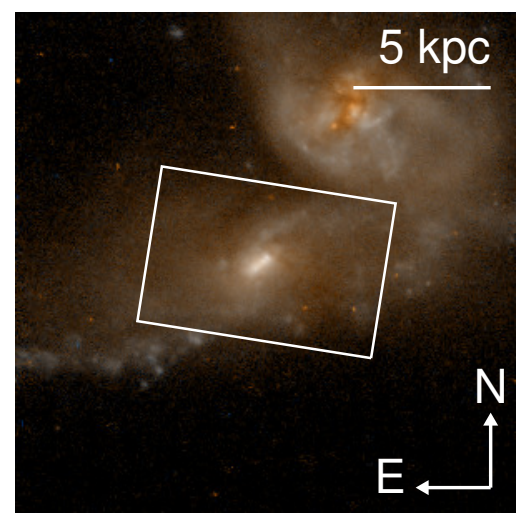

(e) $\log (\mathrm{F} 435 \mathrm{~W} / \mathrm{F} 814 \mathrm{~W})$

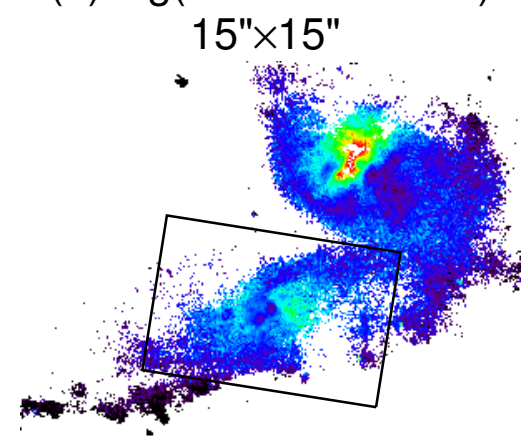

(c) $6100-6800 \AA$

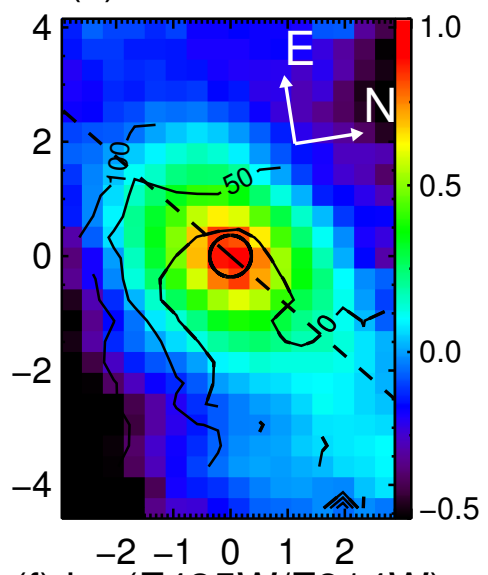

(f) $\log (F 435 W / F 814 W)$ GMOS FOV

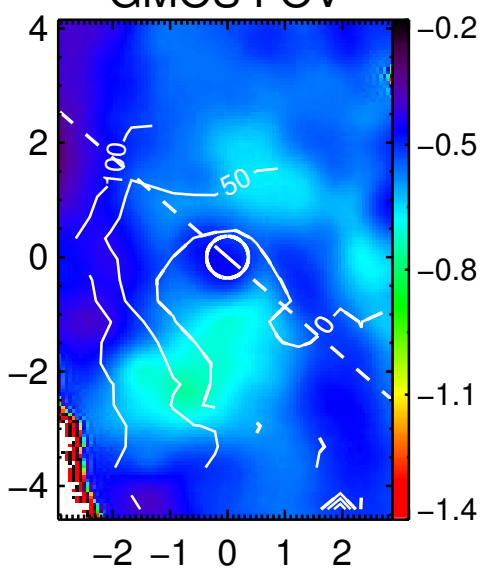

Figure 6. $(a)-(b)$ : Two-color HST image of F08572+3915:SE at sizes $1^{\prime} \times 1^{\prime}$ and $15^{\prime \prime} \times 15^{\prime \prime}$. (See Figure 1 for more details.) (c) Logarithm of total GMOS flux integrated from 6100 to $6800 \AA$, in arbitrary units. See Figure 1 for more details. Velocities are relative to $z_{\text {sys }}=0.0585$. The dashed line is along the major axis of the galaxy bulge, with $\mathrm{PA}=140^{\circ} \mathrm{E}$ of $\mathrm{N}$. $(d)-(e)$ Maps of the $H S T$ continuum flux ratio, $\log (\mathrm{F} 435 \mathrm{~W} / \mathrm{F} 814 \mathrm{~W})$, with sizes and orientations identical to $(a)$ and $(b)$, respectively. $(f)$ Map of $H S T$ color within the GMOS FOV, rotated and smoothed with a Gaussian kernel of FWHM $=1^{\prime \prime}$. 

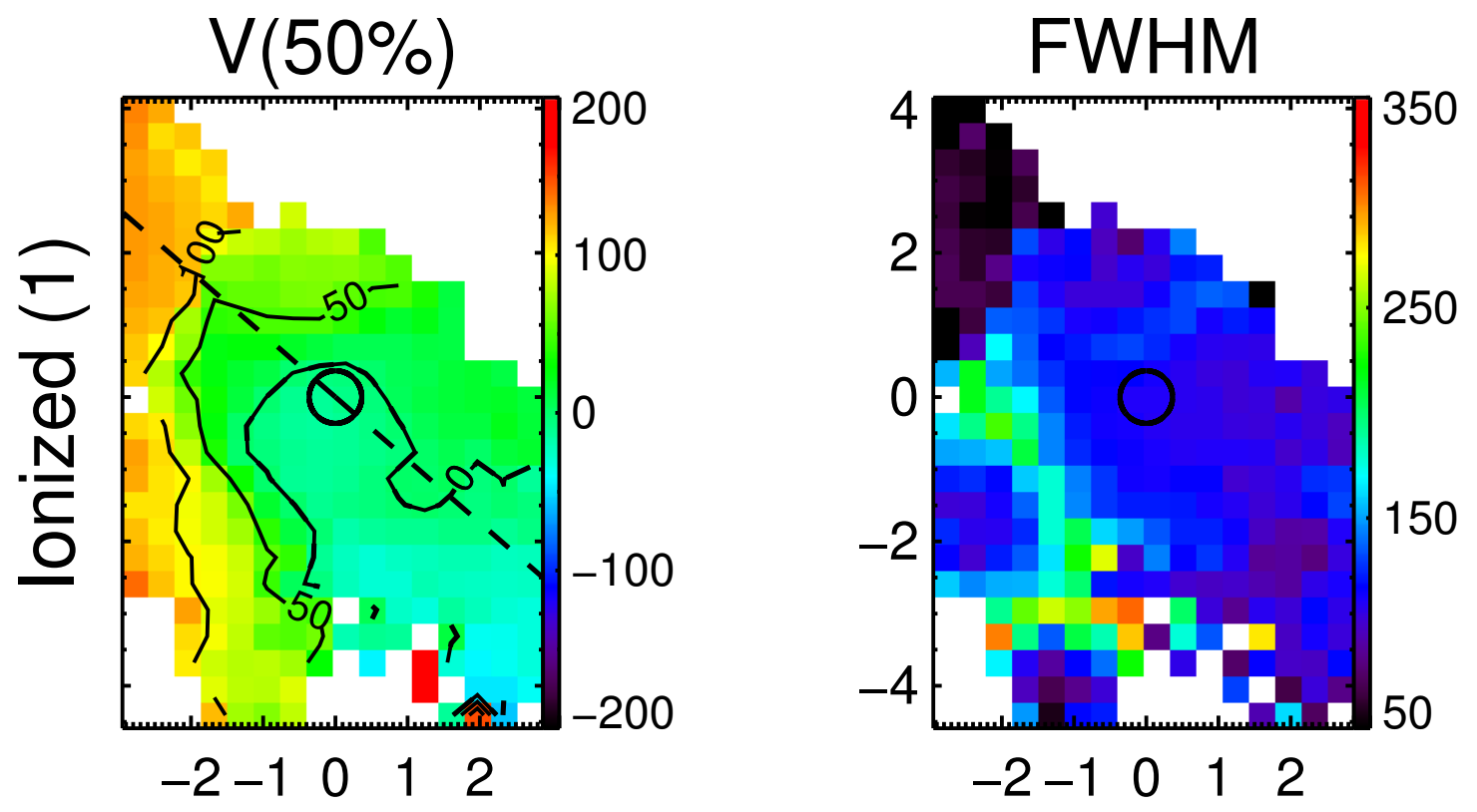

Figure 7. The same as Figure 3 but for F08572+3915:SE.
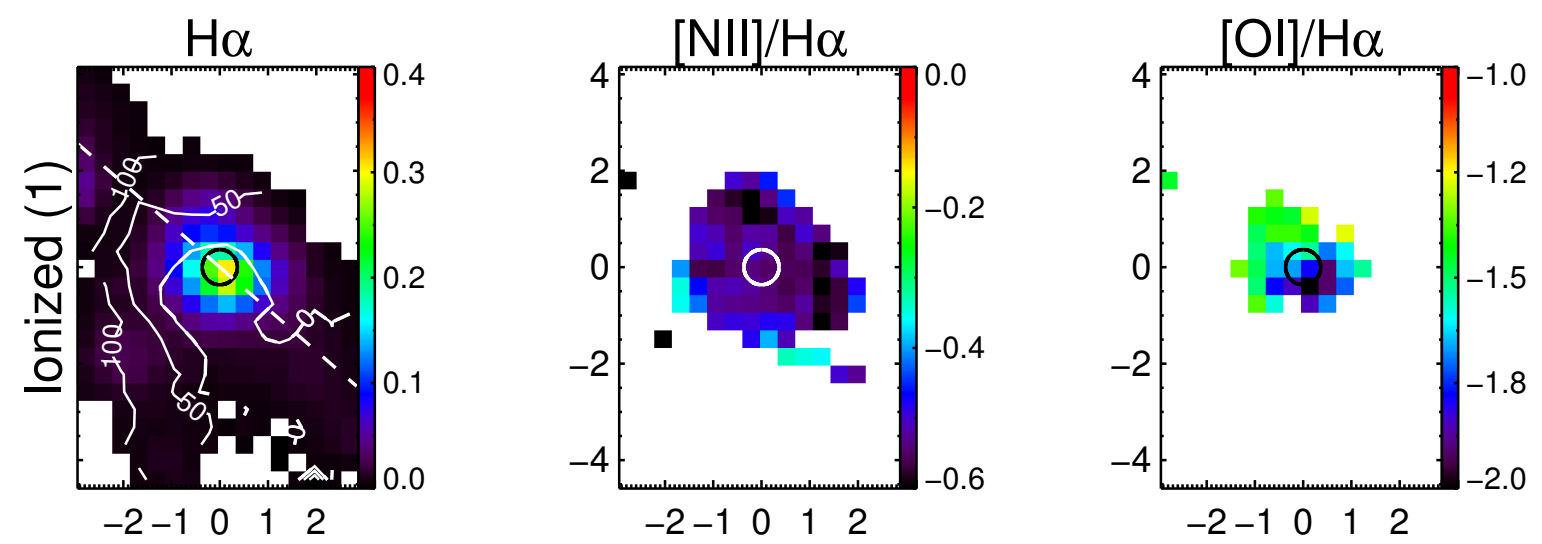

Figure 8. The same as Figure 4 but for F08572+3915:SE. 


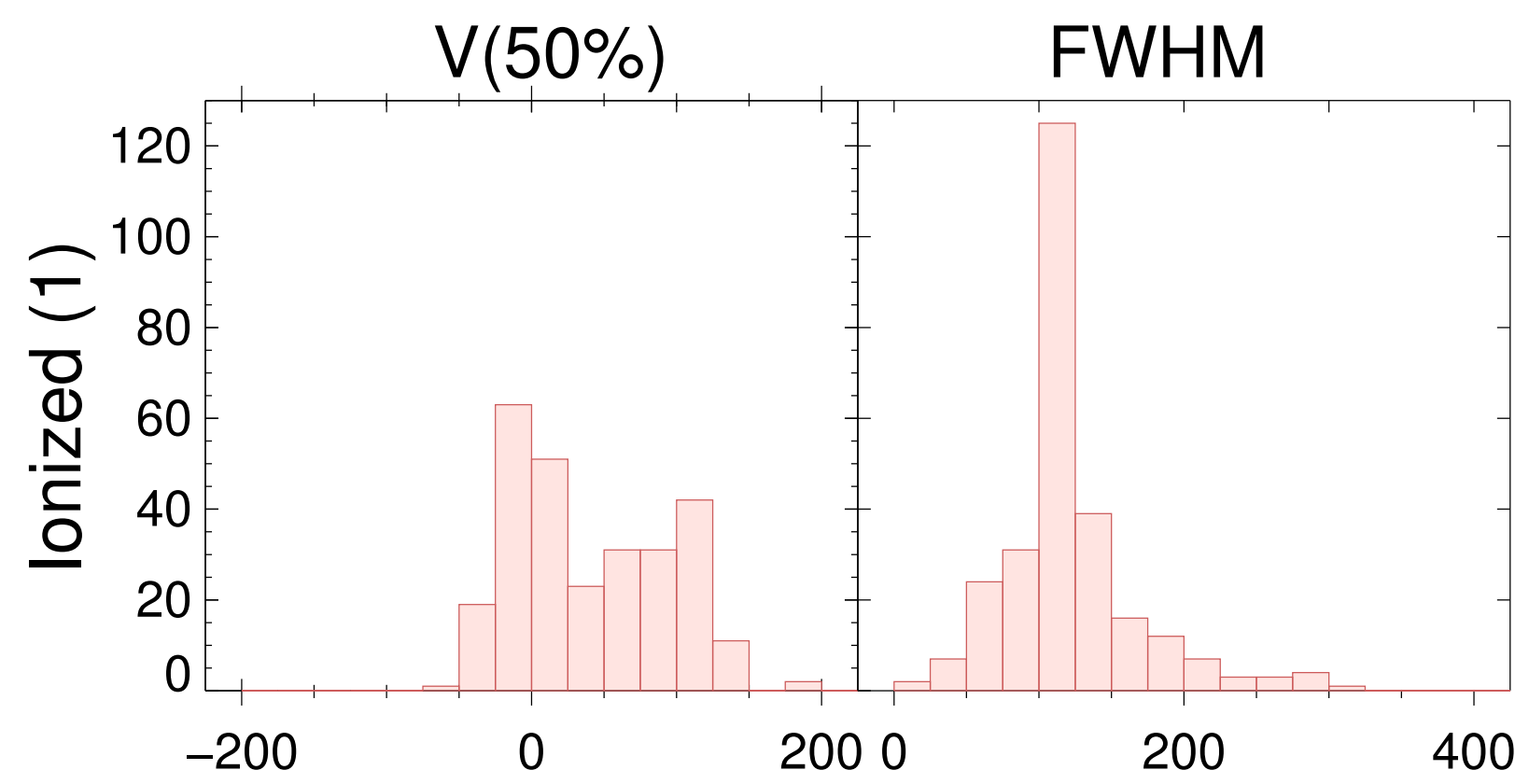

Figure 9. The same as Figure 5 but for F08572+3915:SE.

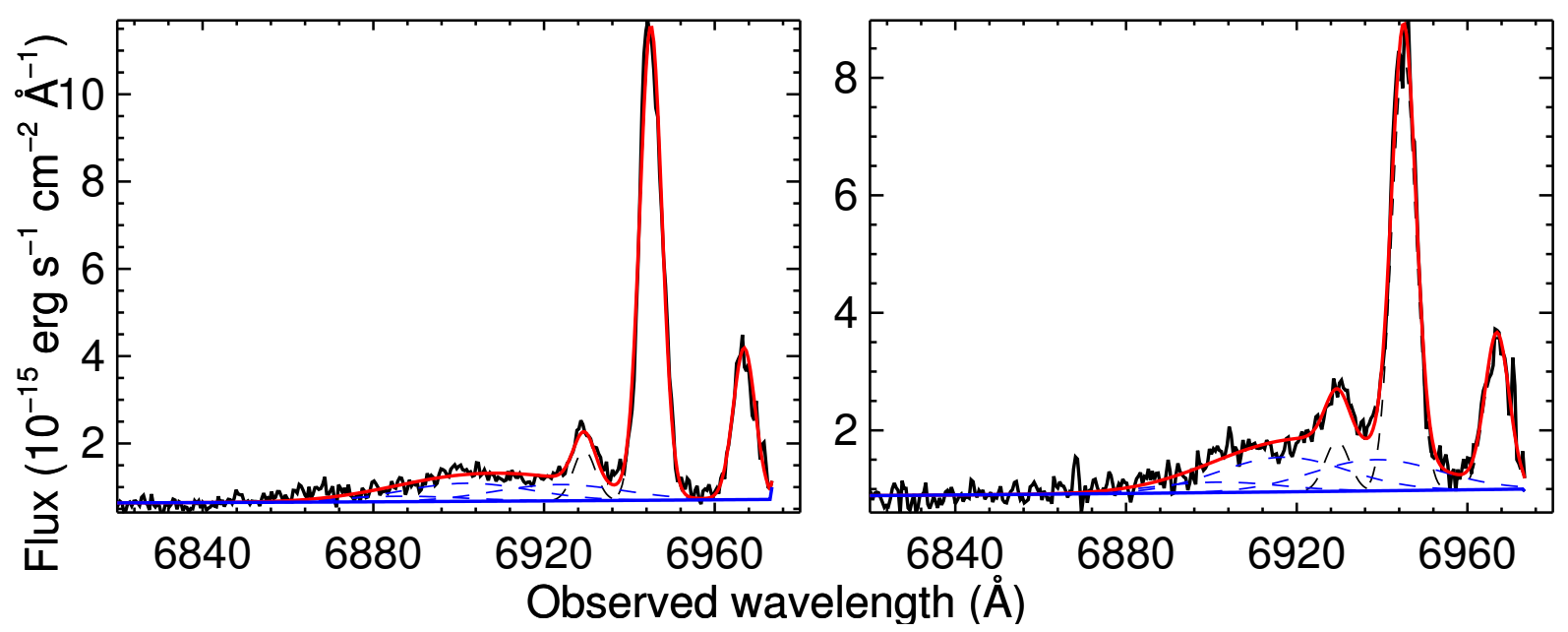

Figure 10. $\mathrm{H} \alpha+[\mathrm{N} \mathrm{II}] 6548,6583 \AA$ spectra of two spaxels in the emission-line outflow of F08572+3915:NW. The solid black line is the data, the solid red line the total fit, the solid blue line the continuum fit, and the dashed lines fits to individual lines. The different colors of the dashed lines represent different velocity components. 


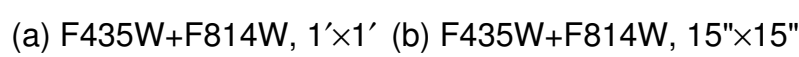

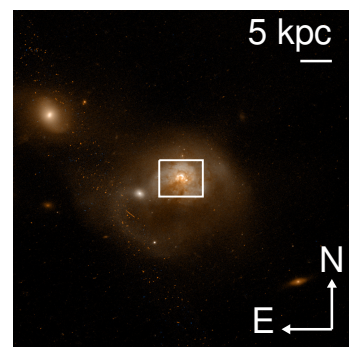

(e) $\log (\mathrm{F} 435 \mathrm{~W} / \mathrm{F} 814 \mathrm{~W})$ $1^{\prime} \times 1^{\prime}$

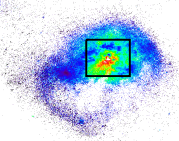

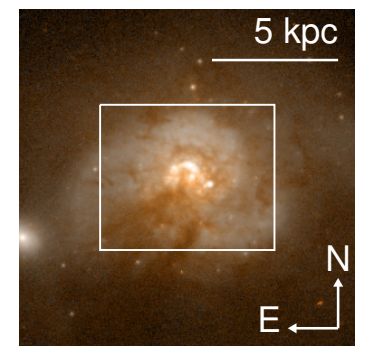

(f) $\log (F 435 W / F 814 W)$ $15 " \times 15^{\prime \prime}$

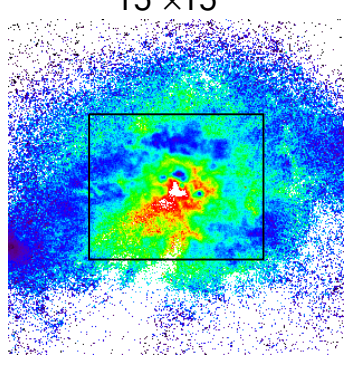

(c) $6100-6800 \AA$

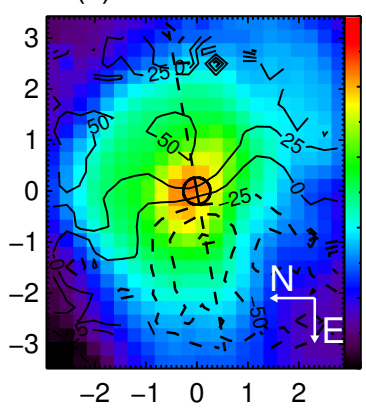

(g) $\log (\mathrm{F} 435 \mathrm{~W} / \mathrm{F} 814 \mathrm{~W})$ GMOS FOV

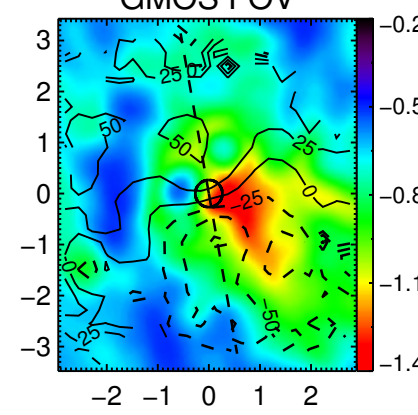

(d) $\mathrm{W}_{\mathrm{NaD}}(\AA)$

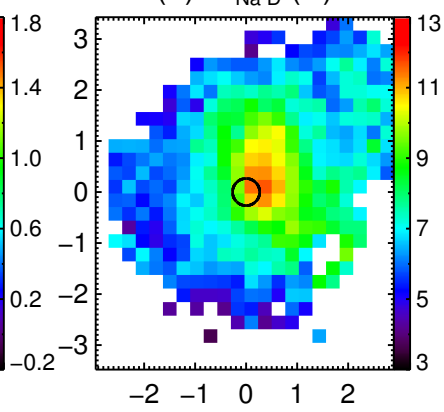

(h) $\mathrm{W}_{\mathrm{NaD}} / \delta \mathrm{W}_{\mathrm{NaD}}$

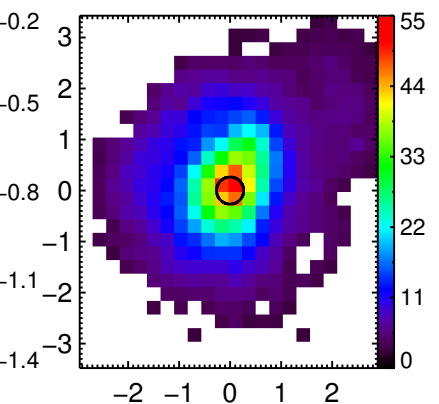

Figure 11. The same as Figure 1 but for F10565+2448. Velocities are relative to $z_{s y s}=0.0431$. The dashed line is along the CO line of nodes (Downes \& Solomon $1998 \mathrm{PA}=100^{\circ} \mathrm{E}$ of $\mathrm{N}$ ). 

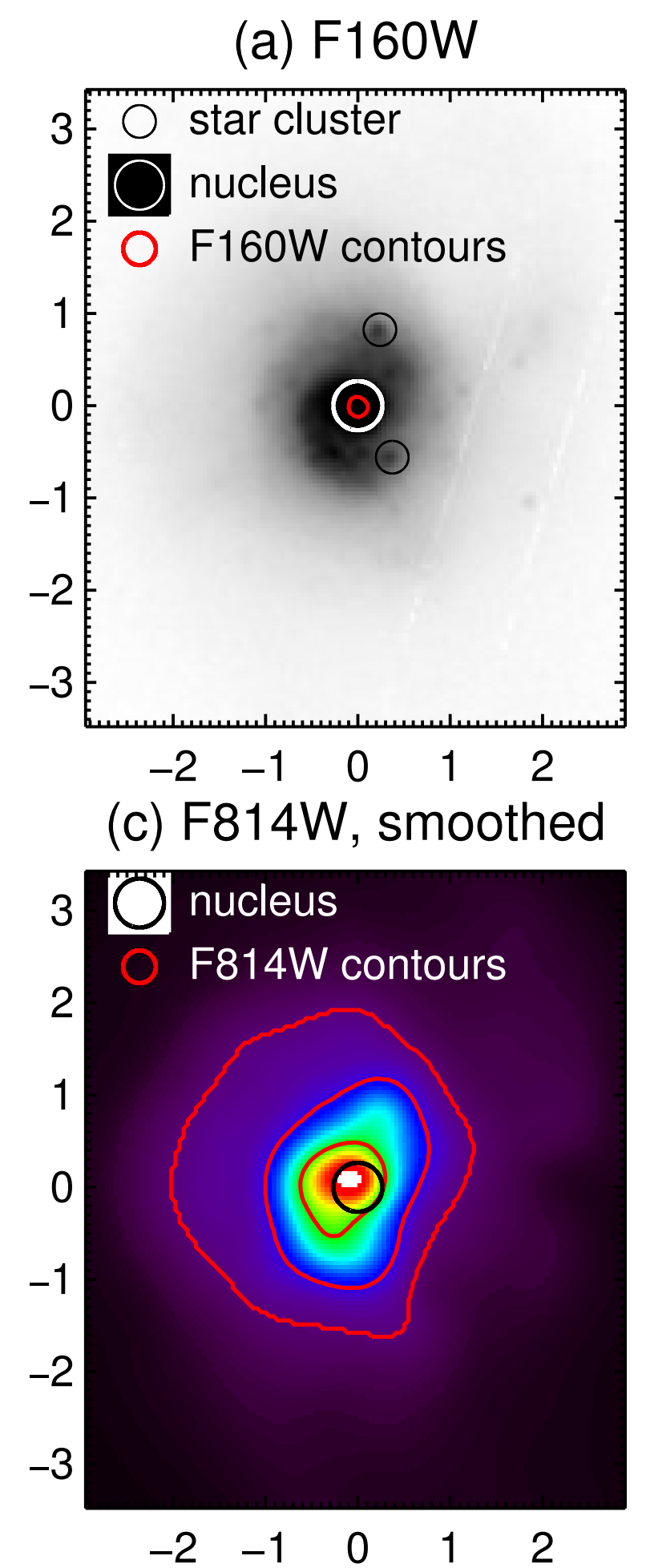

Figure 12. The same as Figure 2 but for F10565+2448. (b) F814W
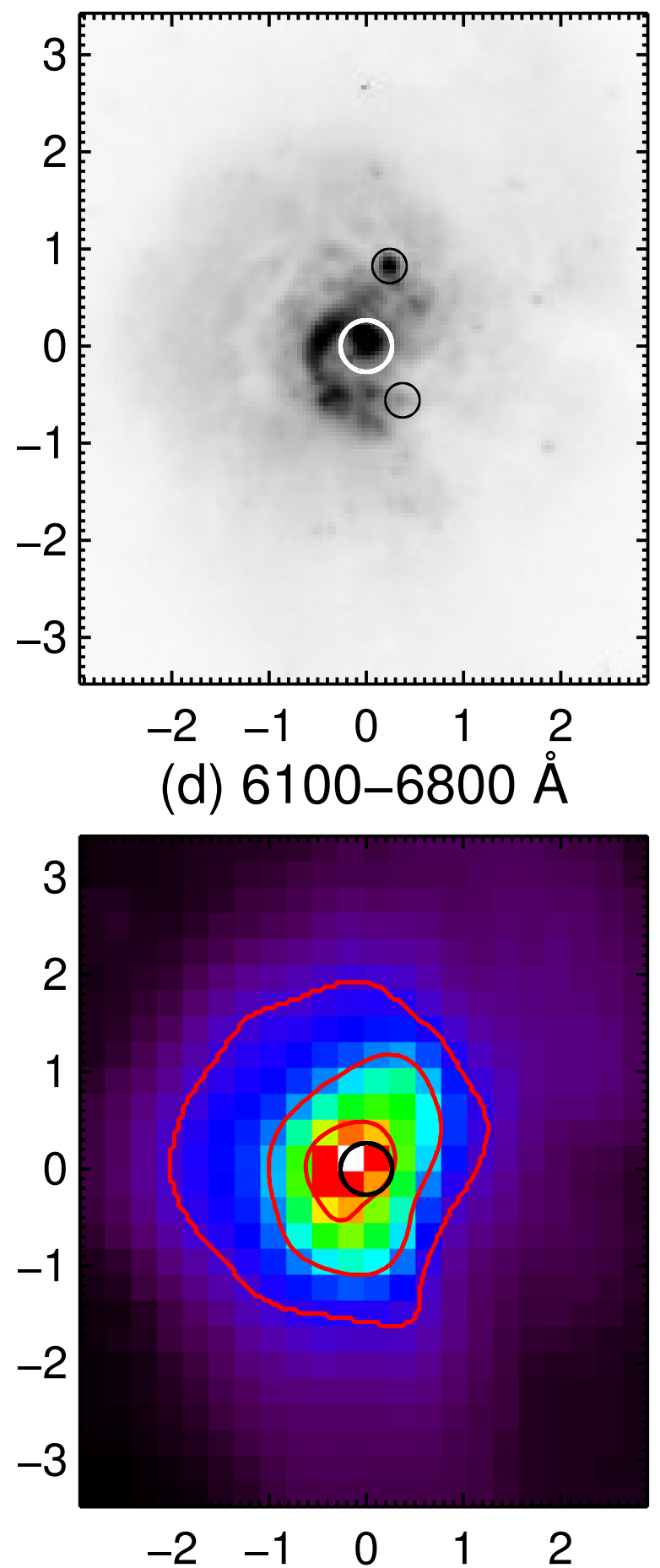

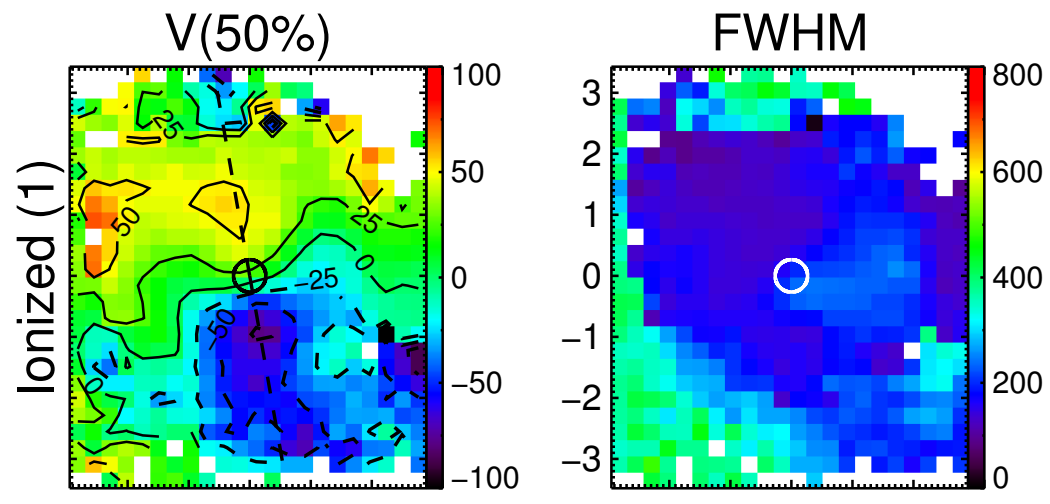

$\mathrm{V}(98 \%)$
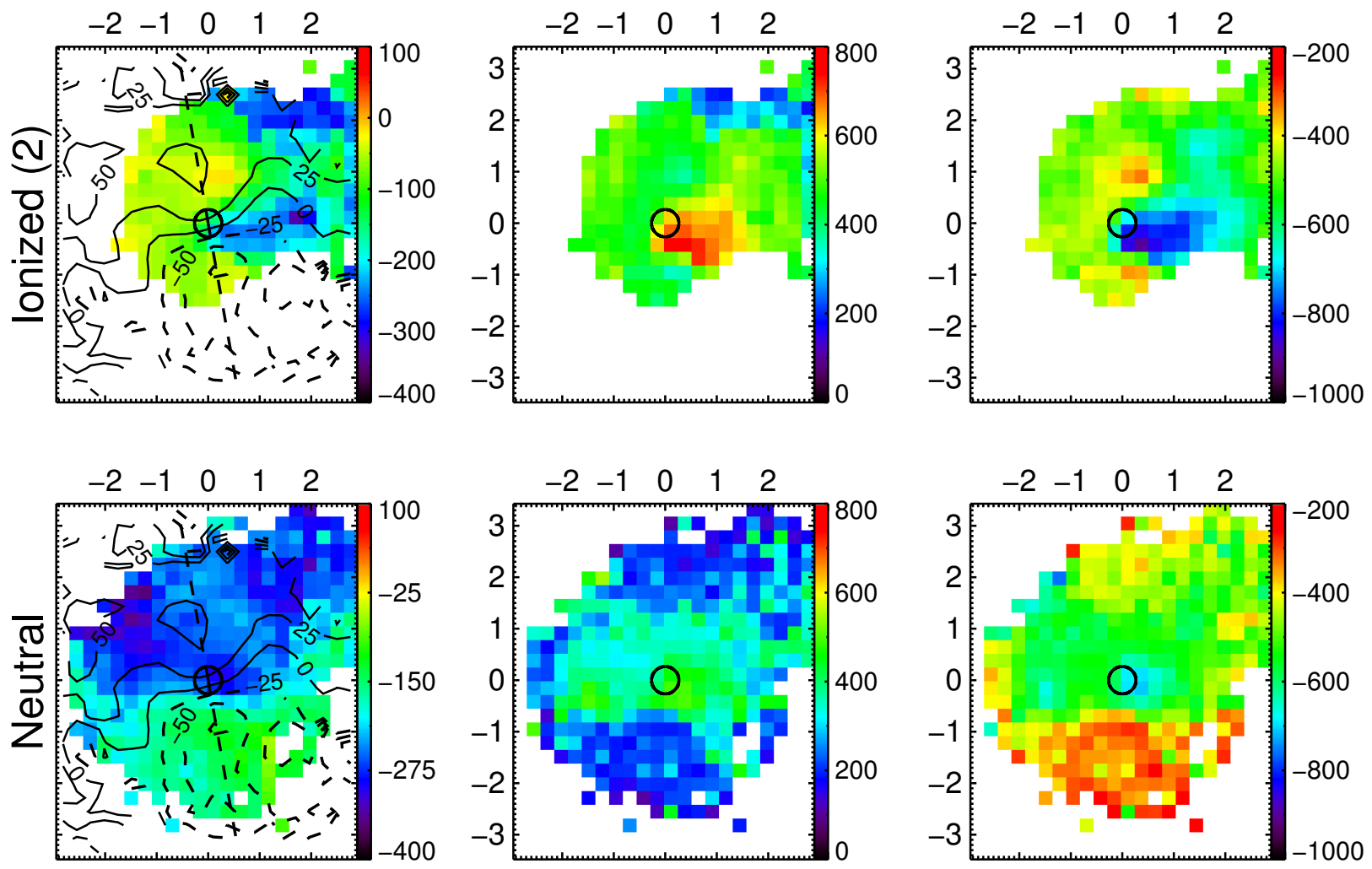

Figure 13. The same as Figure 3 but for F10565+2448. 

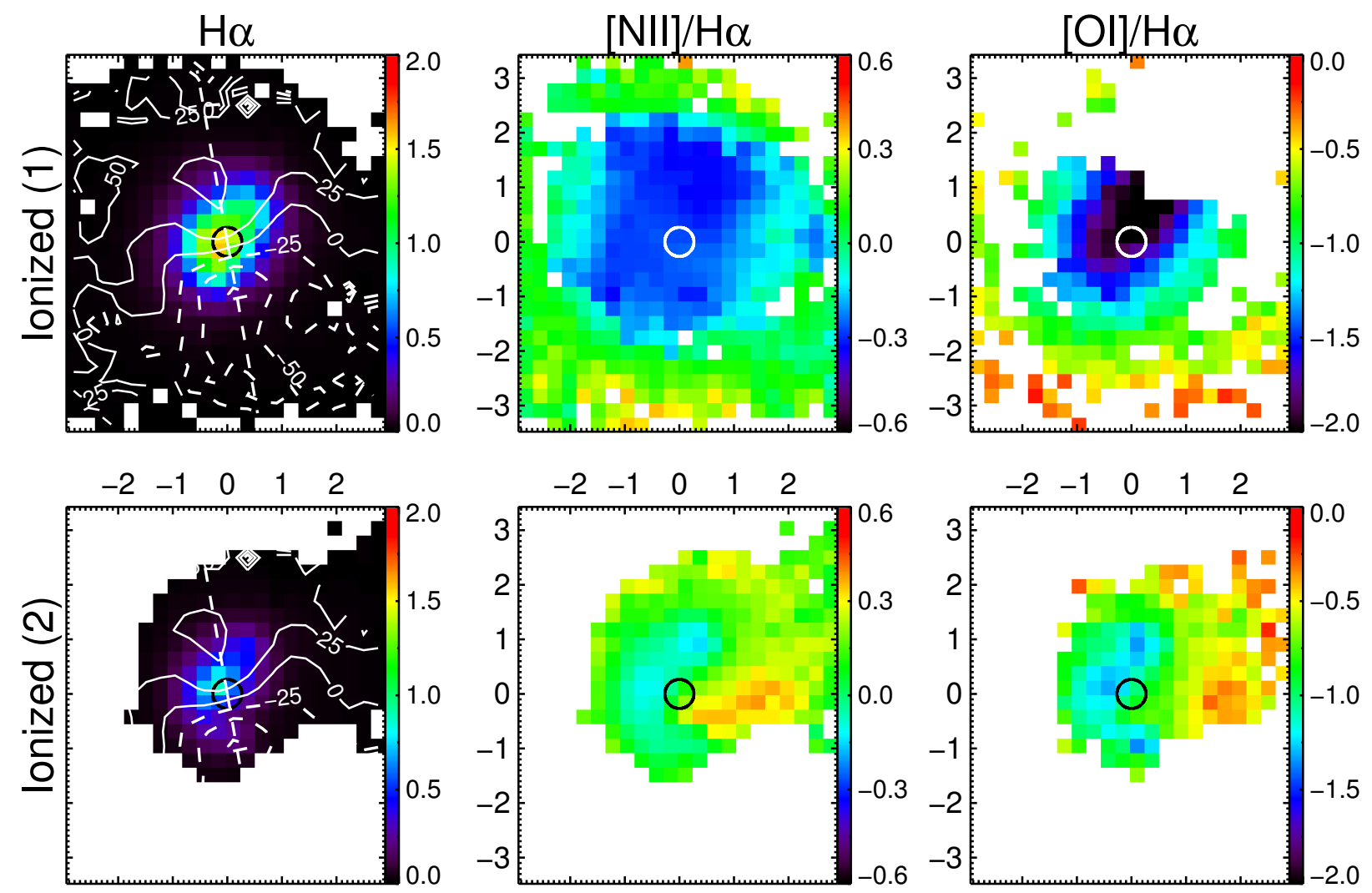

Figure 14. The same as Figure 4 but for F10565+2448. 


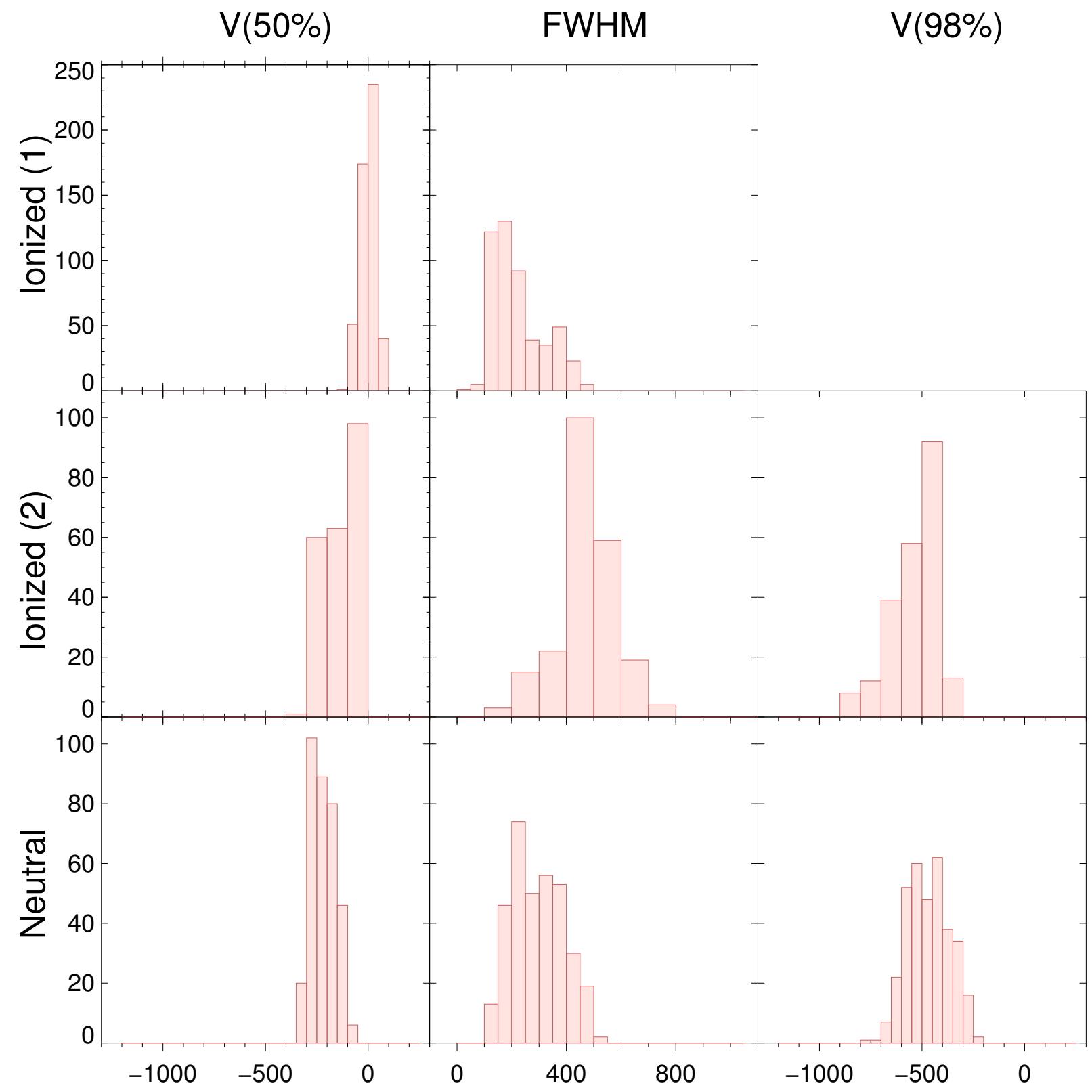

Figure 15. The same as Figure 5 but for F10565+2448. 


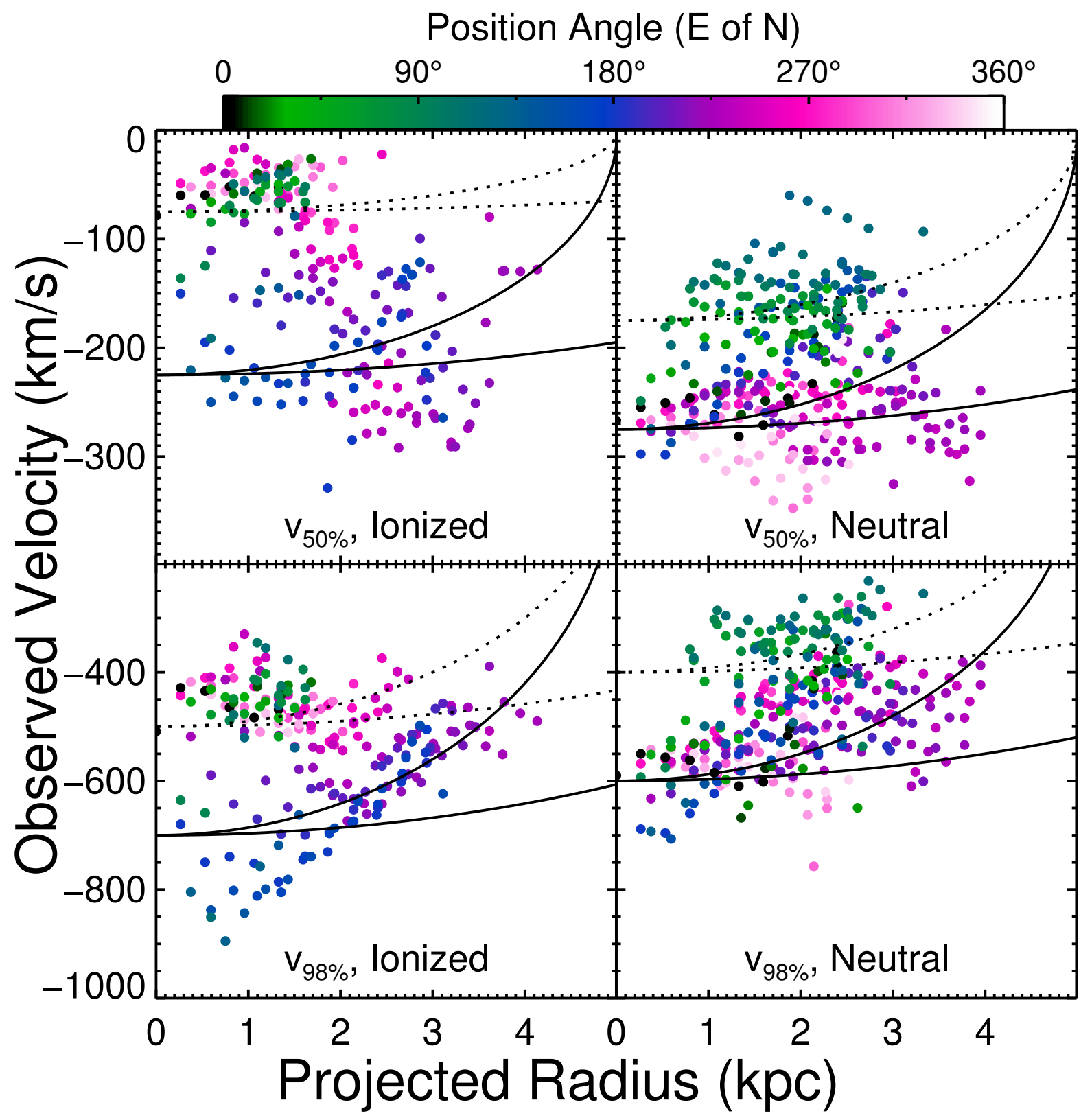

Figure 16. Observed velocity $\left(v_{50 \%}\right.$ and $\left.v_{98} \%\right)$ vs. projected galactocentric radius in the outflowing ionized (broad component only) and neutral gas of F10565+2448. Each point represents one spaxel, and the position angle (E of $\mathrm{N}$ ) of the spaxel with respect to the outflow center is represented by its color. The lines represent single radius free wind models $(\S 3.4$ at constant velocity for $R=5 \mathrm{kpc}$ and $10 \mathrm{kpc}$ (the velocity of each model is given by the value at $R_{\text {proj }}=0 \mathrm{kpc}$ ). The flat profiles of $v_{50 \%}$ imply large wind radii $(\geq 5 \mathrm{kpc}$ ), and the strong asymmetry $\mathrm{E}$ and $\mathrm{W}$ of the disk requires asymmetric acceleration. The declining $v_{98 \%}$ values either place an upper limit on the wind radius $(\lesssim 10 \mathrm{kpc})$ or reflect the decrease in unresolved (turbulent) motions with radius. (See $\S 4.2 .2$ for more details.) 
(a) FWHM, lonized
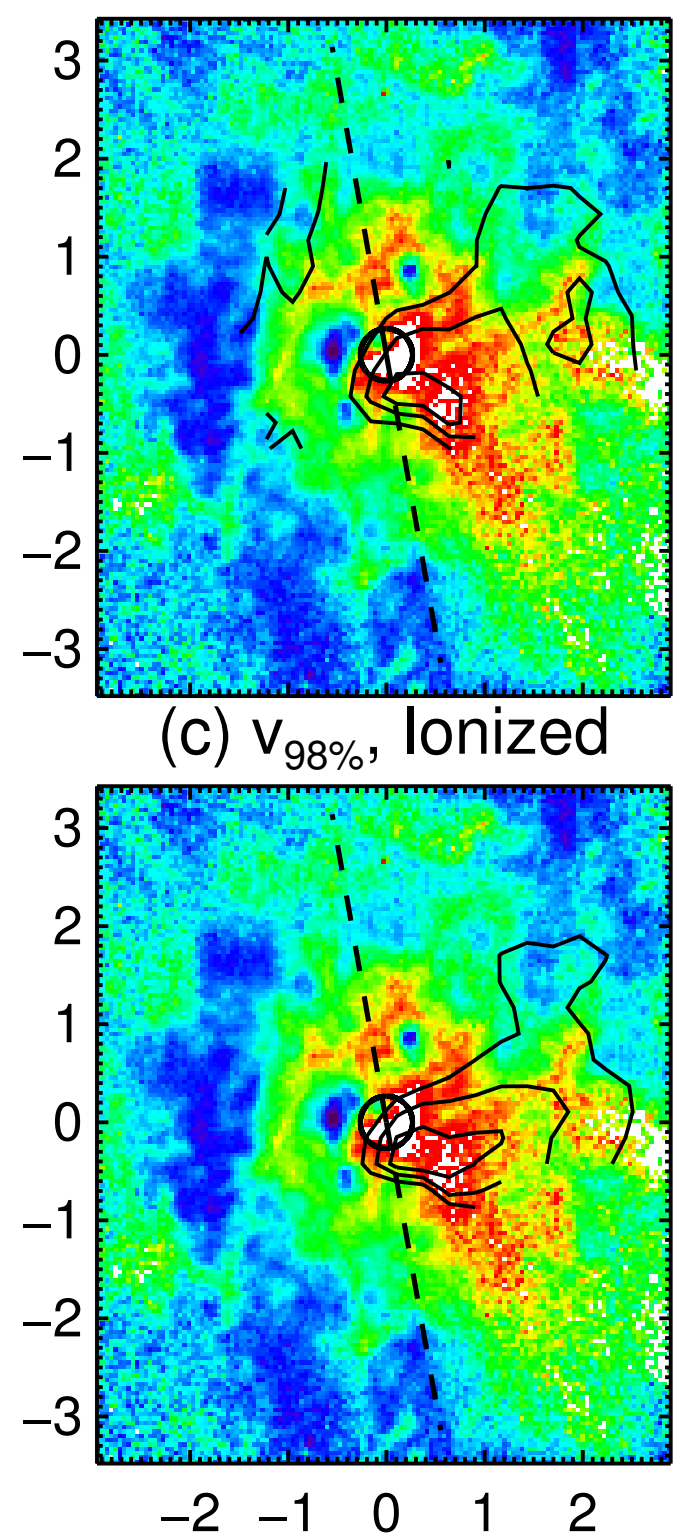

(b) FWHM, Neutral
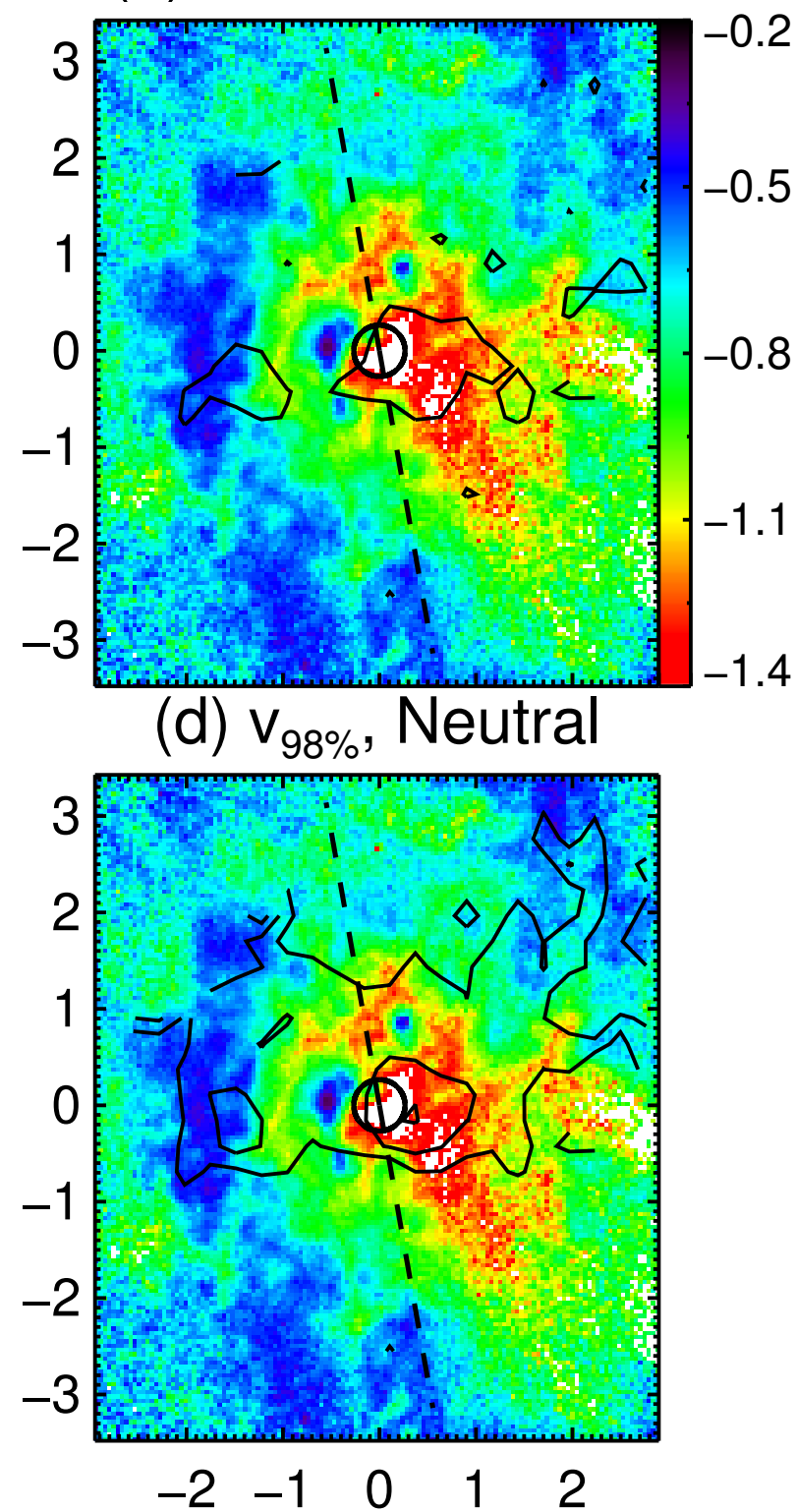

Figure 17. Color maps of the $H S T$ continuum flux ratio, $\log (\mathrm{F} 435 \mathrm{~W} / \mathrm{F} 814 \mathrm{~W})$, in $\mathrm{F} 10565+2448$, with velocity contours overlaid: (a) ionized gas FWHM (contours: 500, 600, $700 \mathrm{~km} \mathrm{~s}^{-1}$ ); (b) neutral gas FWHM (contours: $400 \mathrm{~km} \mathrm{~s}^{-1}$ ); (c) ionized gas $v_{98 \%}$ (contours: $\left.-800,-700,-600 \mathrm{~km} \mathrm{~s}^{-1}\right)$; and (d) neutral gas $v_{98 \%}$ (contours: $-700,-600,-500 \mathrm{~km} \mathrm{~s}^{-1}$ ). The dashed line represents the position angle of the CO disk (Downes \& Solomon 1998), and the open circle the GMOS continuum peak. The relative color and morphology of the blue clumps to the lett (N) are consistent with unobscured star forming regions in the near side of a galaxy disk, while the dusty, high-velocity gas to the right $(\mathrm{S})$ is almost certainly a minor-axis outflow. The minor-axis outflow reveals dusty filaments and unresolved motions of hundreds of $\mathrm{km} \mathrm{s}^{-1}$, consistent with dusty, shocked gas. If this outflow is emerging along the minor axis of the $\mathrm{CO}$ disk, it is inclined $\sim 20^{\circ}$ away from the line of sight (Downes \& Solomon 1998). (See $\S 4.2 .2$ for more details.) 
(a) F435W+F814W, $1^{\prime} \times 1^{\prime}$

(b) $\mathrm{F} 435 \mathrm{~W}+\mathrm{F} 814 \mathrm{~W}, 15 " \times 15 "$

(c) $6100-6800 \AA$

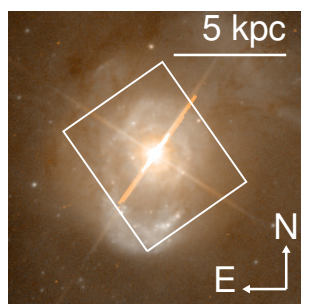

(e) $\log (\mathrm{F} 435 \mathrm{~W} / \mathrm{F} 814 \mathrm{~W})$ $1^{\prime} \times 1^{\prime}$

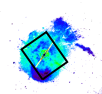

(f) $\log (F 435 W / F 814 W)$
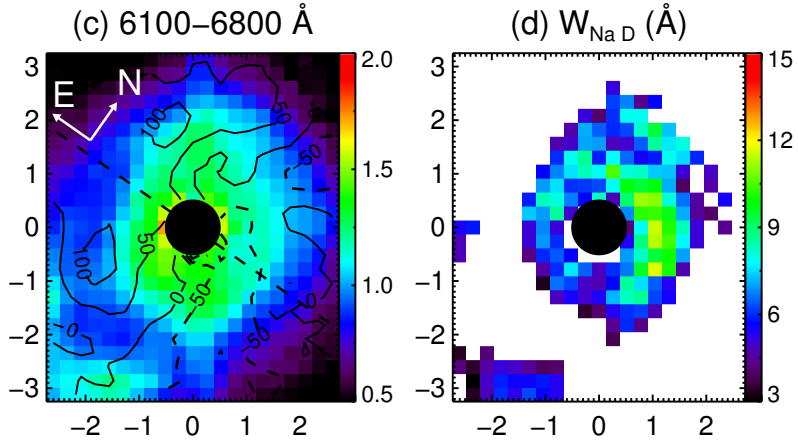

(g) $\log ($ F435W/F814W)

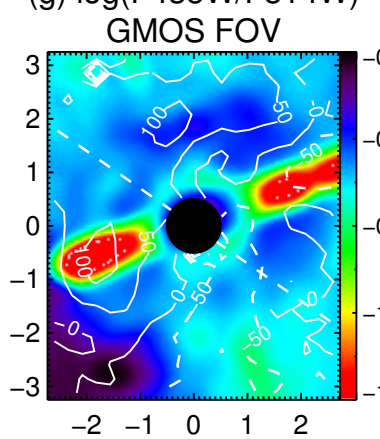

(h) $\mathrm{W}_{\mathrm{NaD}} / \delta \mathrm{W}_{\mathrm{NaD}}$

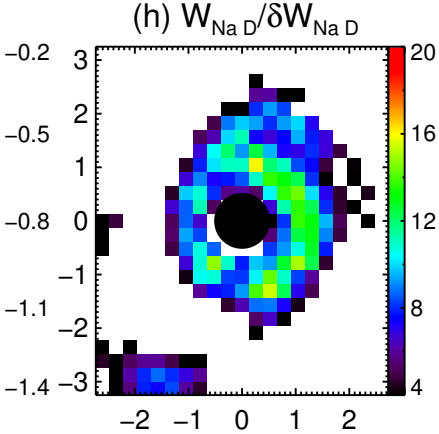

Figure 18. The same as Figure 1 but for Mrk 231. Velocities are relative to $z_{\text {sys }}=0.0422$. The dashed line is along the CO line of nodes (Downes \& Solomon $1998 \mathrm{PA}=9^{\circ} \mathrm{E}$ of $\mathrm{N}$ ). 


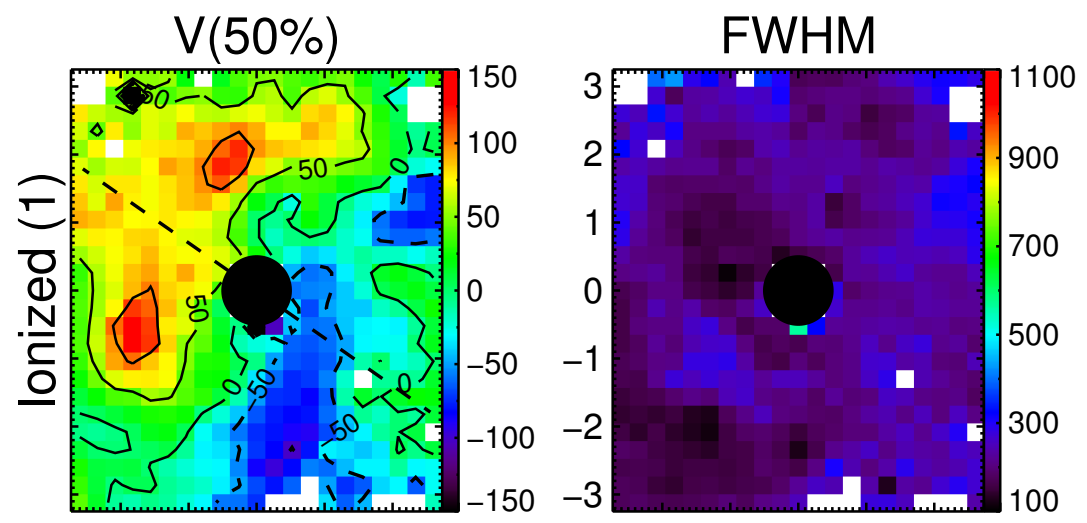

$\mathrm{V}(98 \%)$
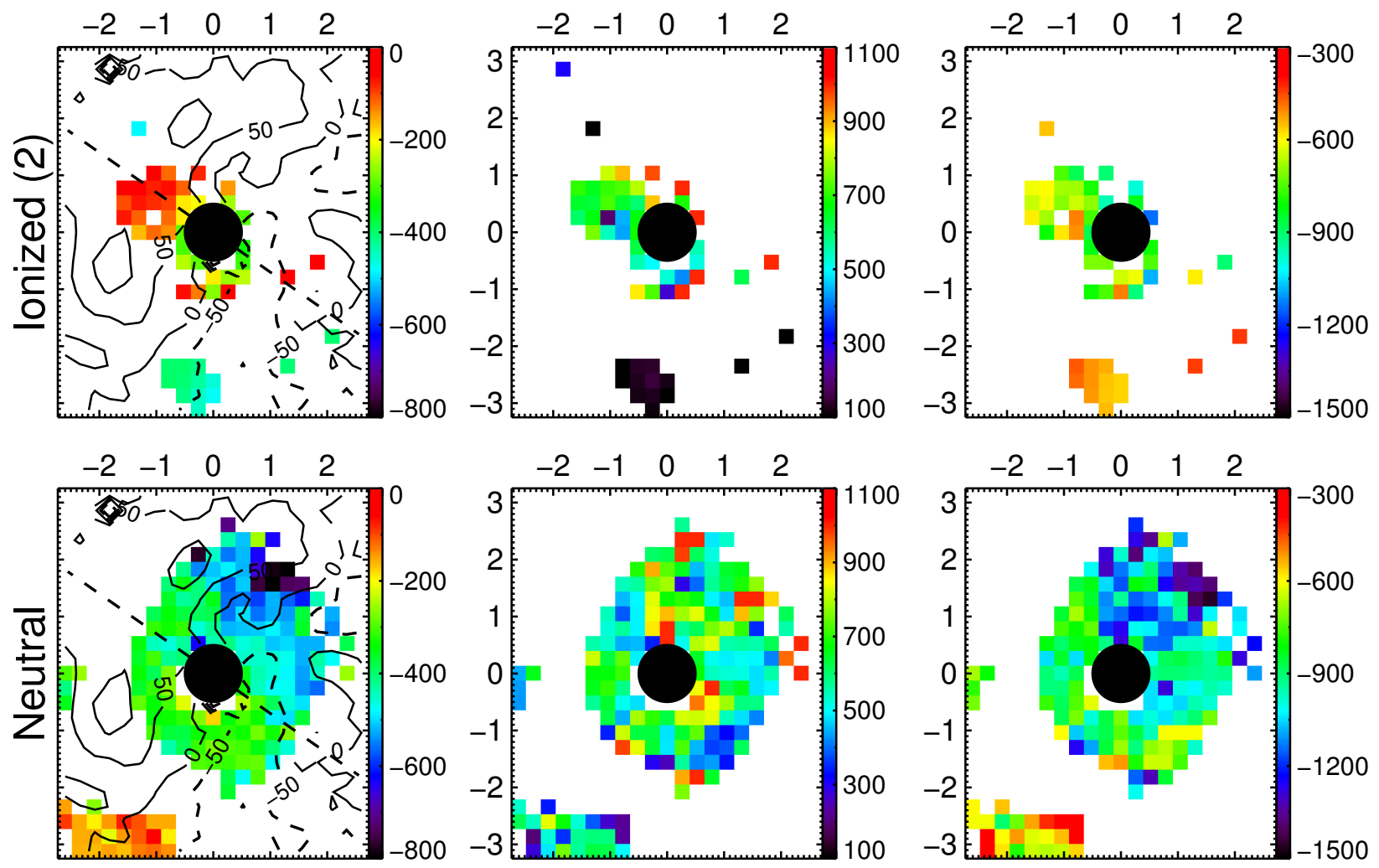

Figure 19. The same as Figure 3 but for Mrk 231. 

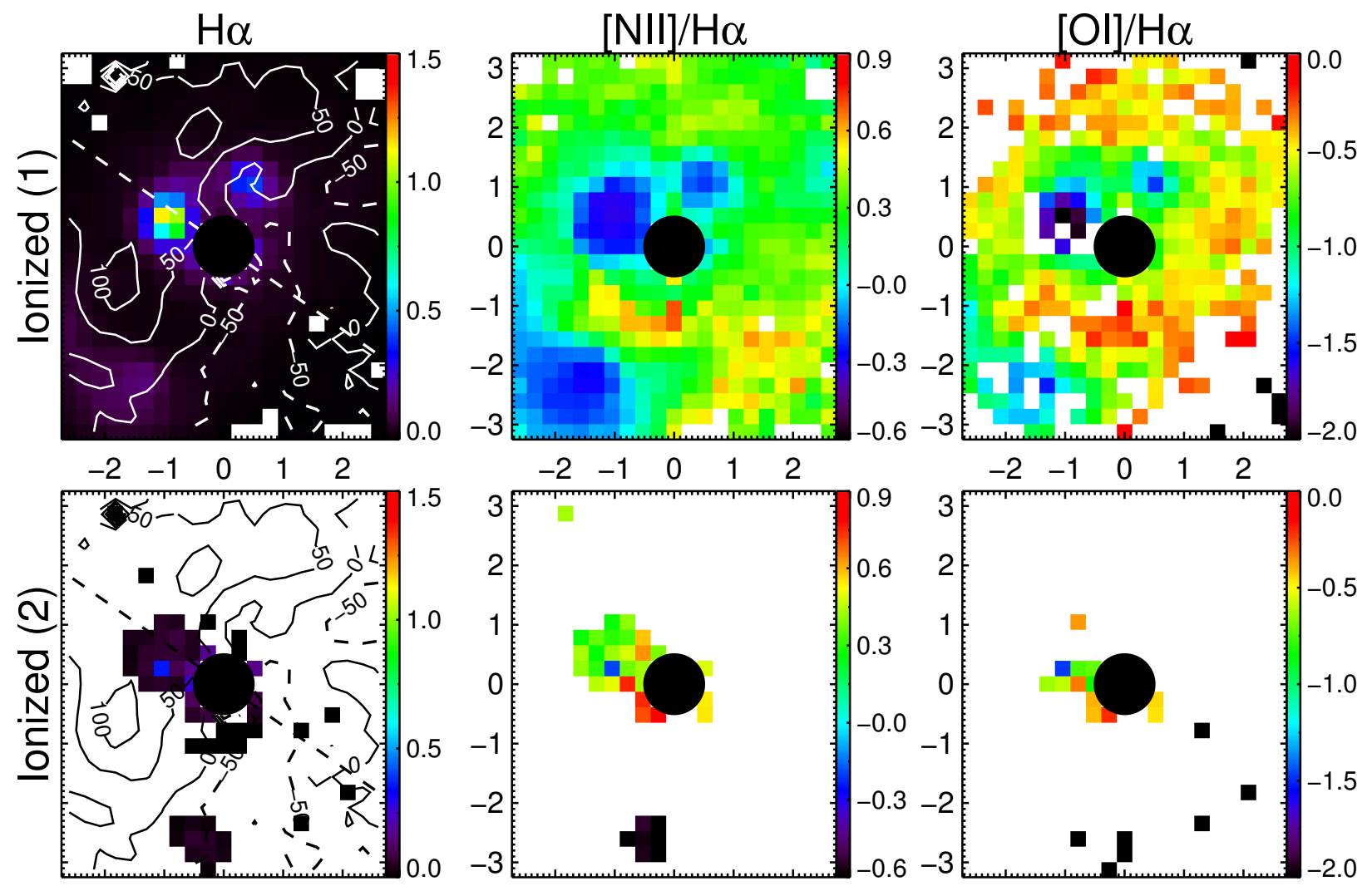

Figure 20. The same as Figure 4 but for Mrk 231. 


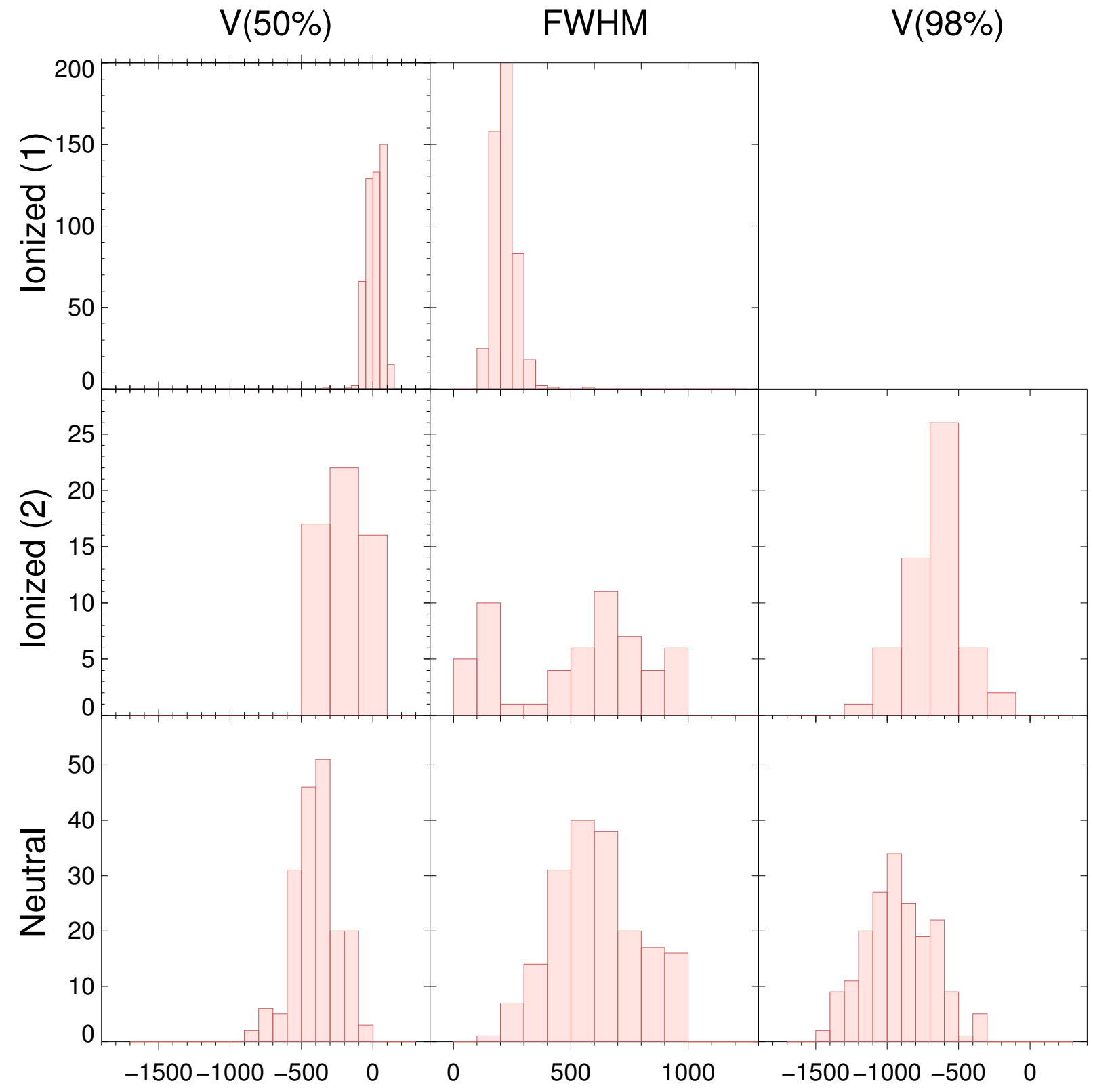

Figure 21. The same as Figure 5 but for Mrk 231. 
(a) F435W+F814W, $1^{\prime} \times 1^{\prime}$

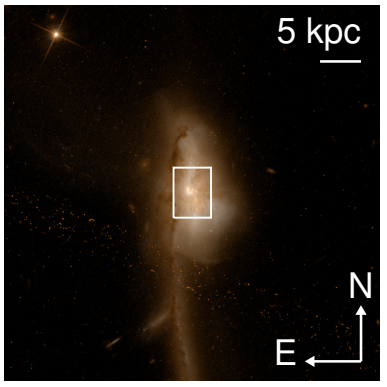

(e) $\log (\mathrm{F} 435 \mathrm{~W} / \mathrm{F} 814 \mathrm{~W})$

$1^{\prime} \times 1^{\prime}$

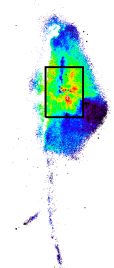

(b) F435W+F814W, 15"×15"

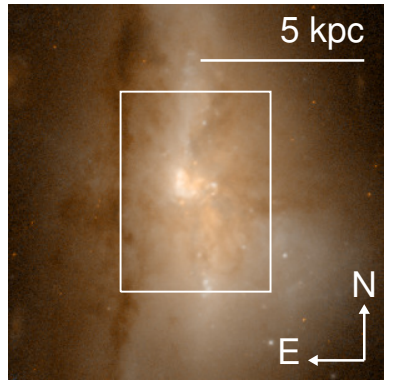

(f) $\log (F 435 W / F 814 W)$

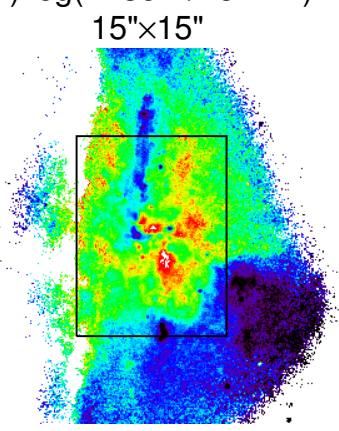

(c) $6100-6800 \AA$

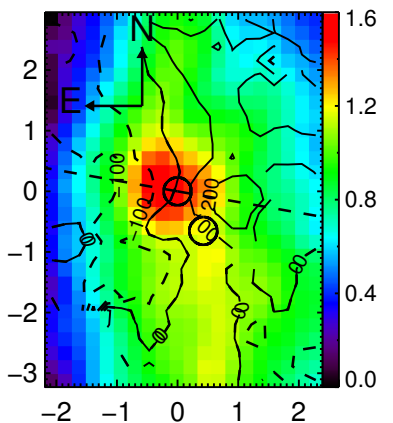

(g) $\log ^{-1}(F 435 W / F 814 W)$

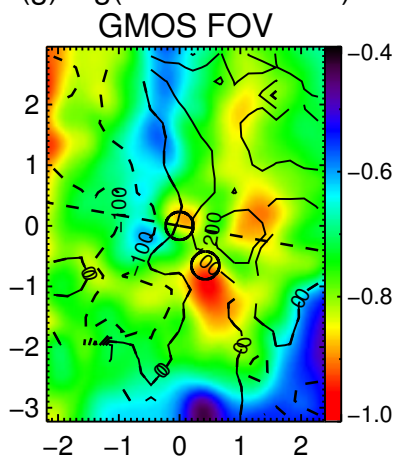

(d) $\mathrm{W}_{\mathrm{Na} \text { D }}(\AA)$

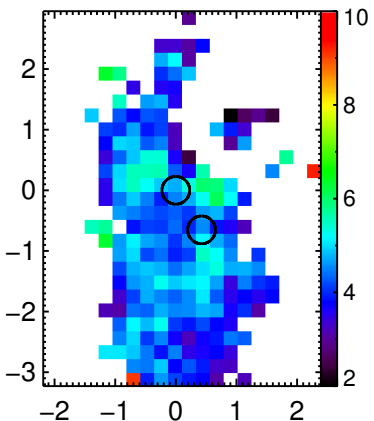

(h) $\mathrm{W}_{\mathrm{Na} D} / \delta \mathrm{W}_{\mathrm{Na} \mathrm{D}}$

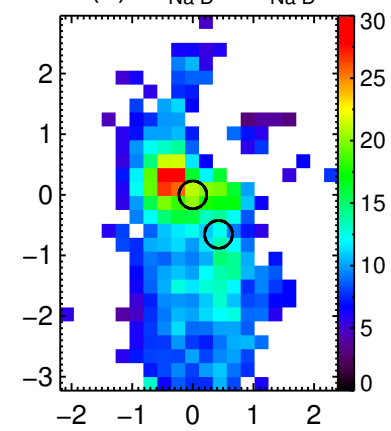

Figure 22. The same as Figure 1, but for Mrk 273. The open circles are the locations of the NIR nuclei. Velocities are relative to $z_{\text {sus }}=0.0373$. The dashed line is along the approximate line of nodes of the gas disk around the $\mathrm{N}$ nucleus (Downes \& Solomon 1998 . Cole et al. 1999, Wilson et al. 2008, PA $\sim 80^{\circ} \mathrm{E}$ of $\mathrm{N}$ ). 


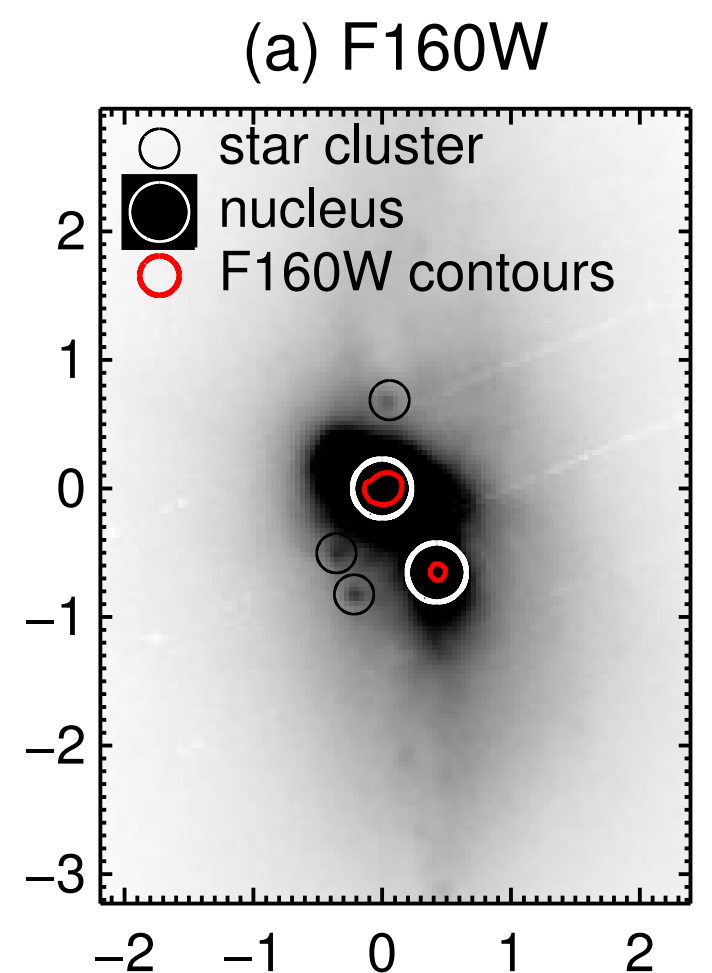

(c) F814W, smoothed

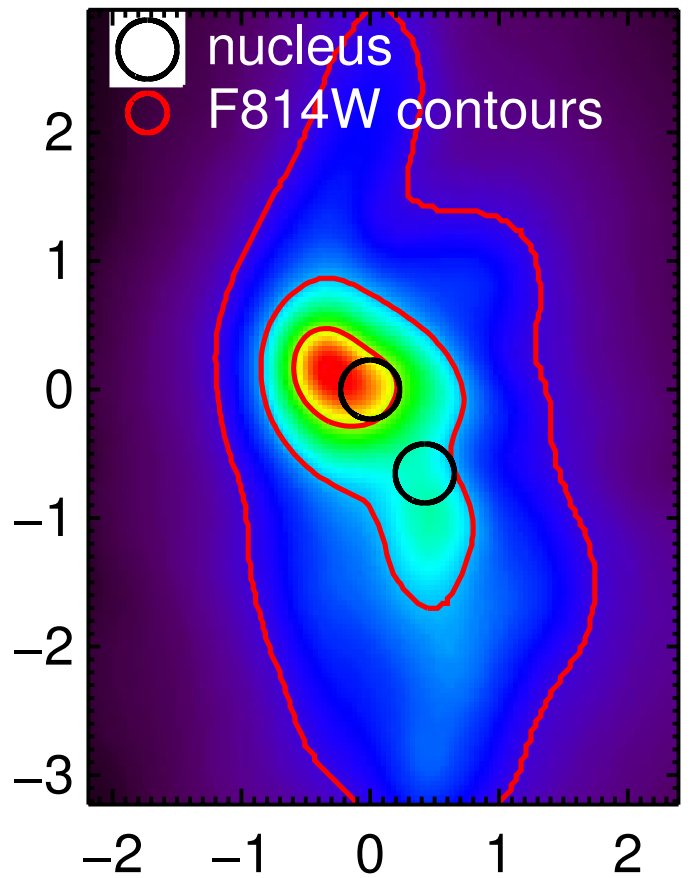

(b) F814W
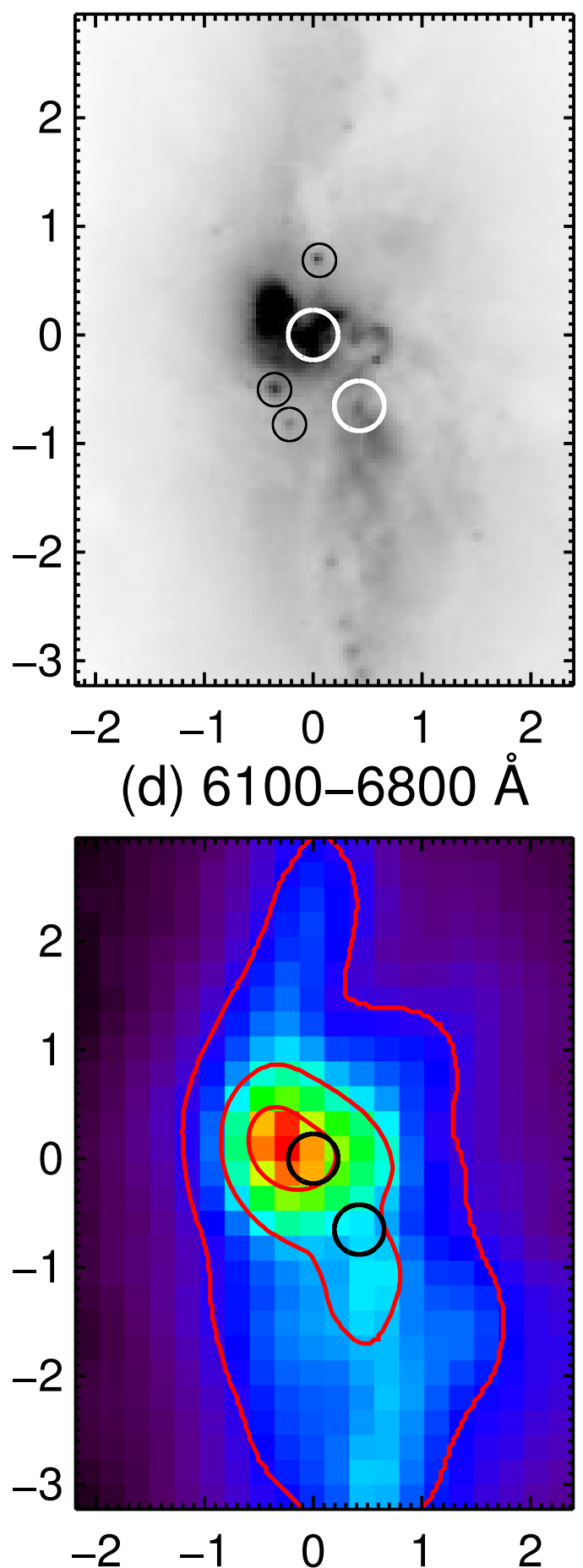

Figure 23. The same as Figure 2 but for Mrk 273. 

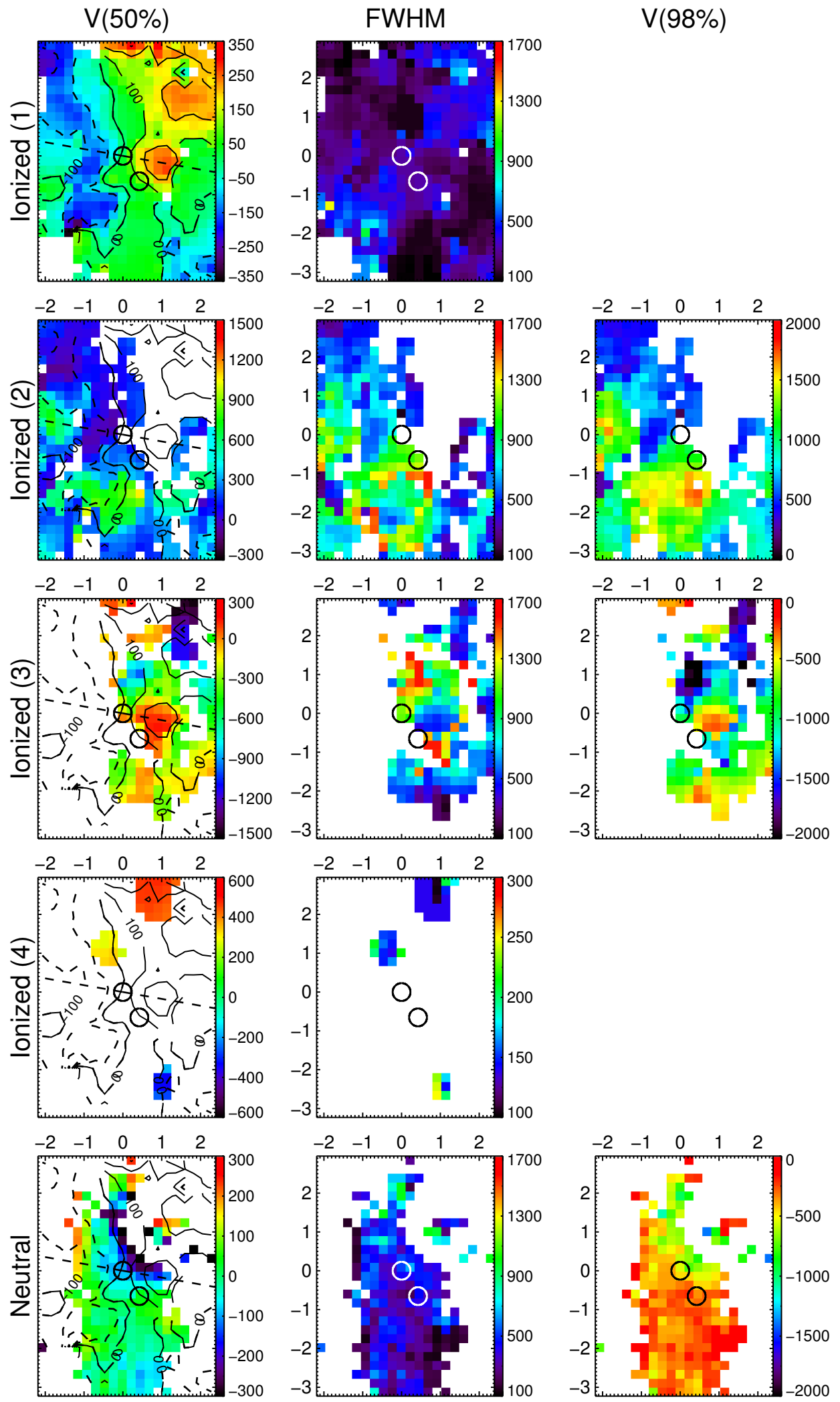

Figure 24. The same as Figure 3 but for Mrk 273. For this sytem, the four ionized gas components are: (1) rotating, (2) broad and redshifted, (3) broad and blueshifted, and (4) isolated clouds with narrow profiles $\left(\$ 4.4 .2\right.$. For component $2, v_{98 \%}$ is computed by adding, rather than subtracting, $2 \sigma(\S 3.3$. 
$\mathrm{H} \alpha$
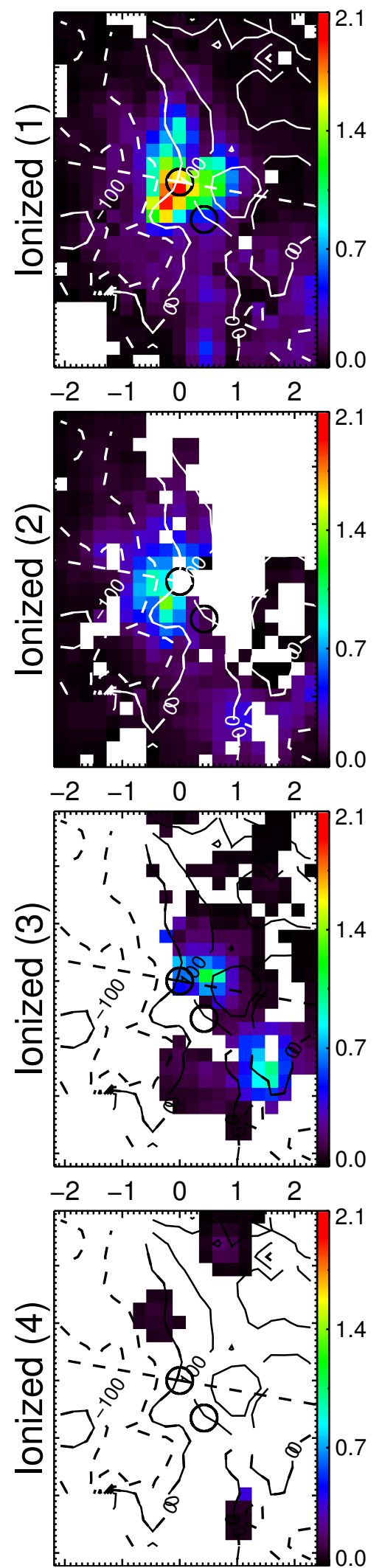

$[\mathrm{NII} / \mathrm{H} \alpha$
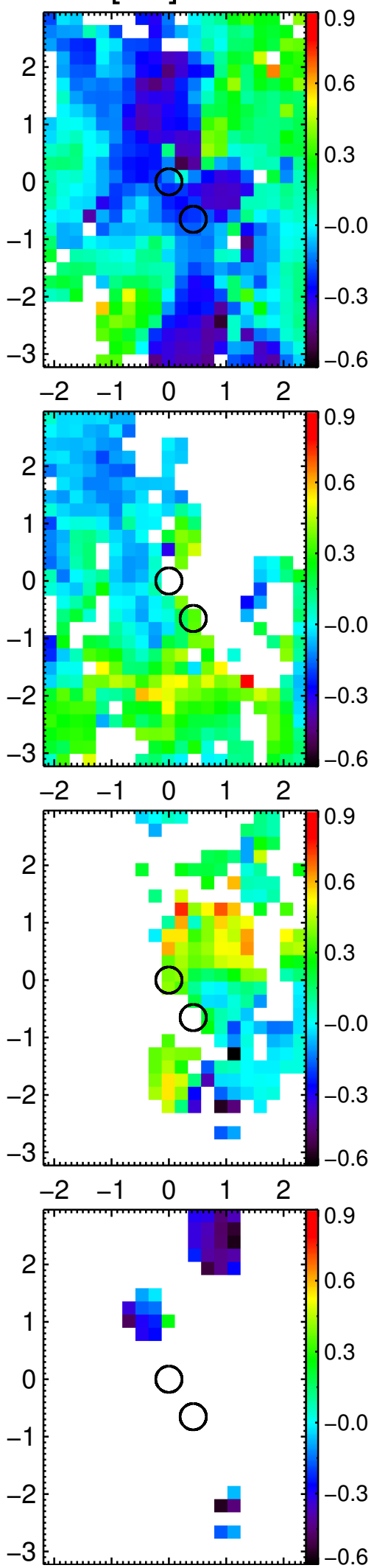

[Ol] $/ \mathrm{H} \alpha$
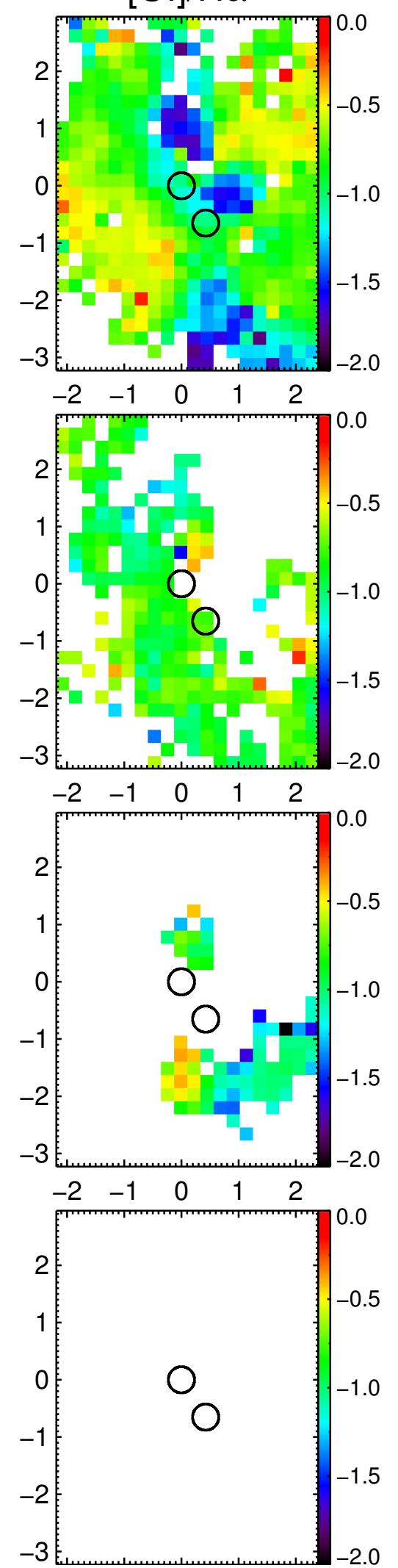

Figure 25. The same as Figure 4 but for Mrk 273. 

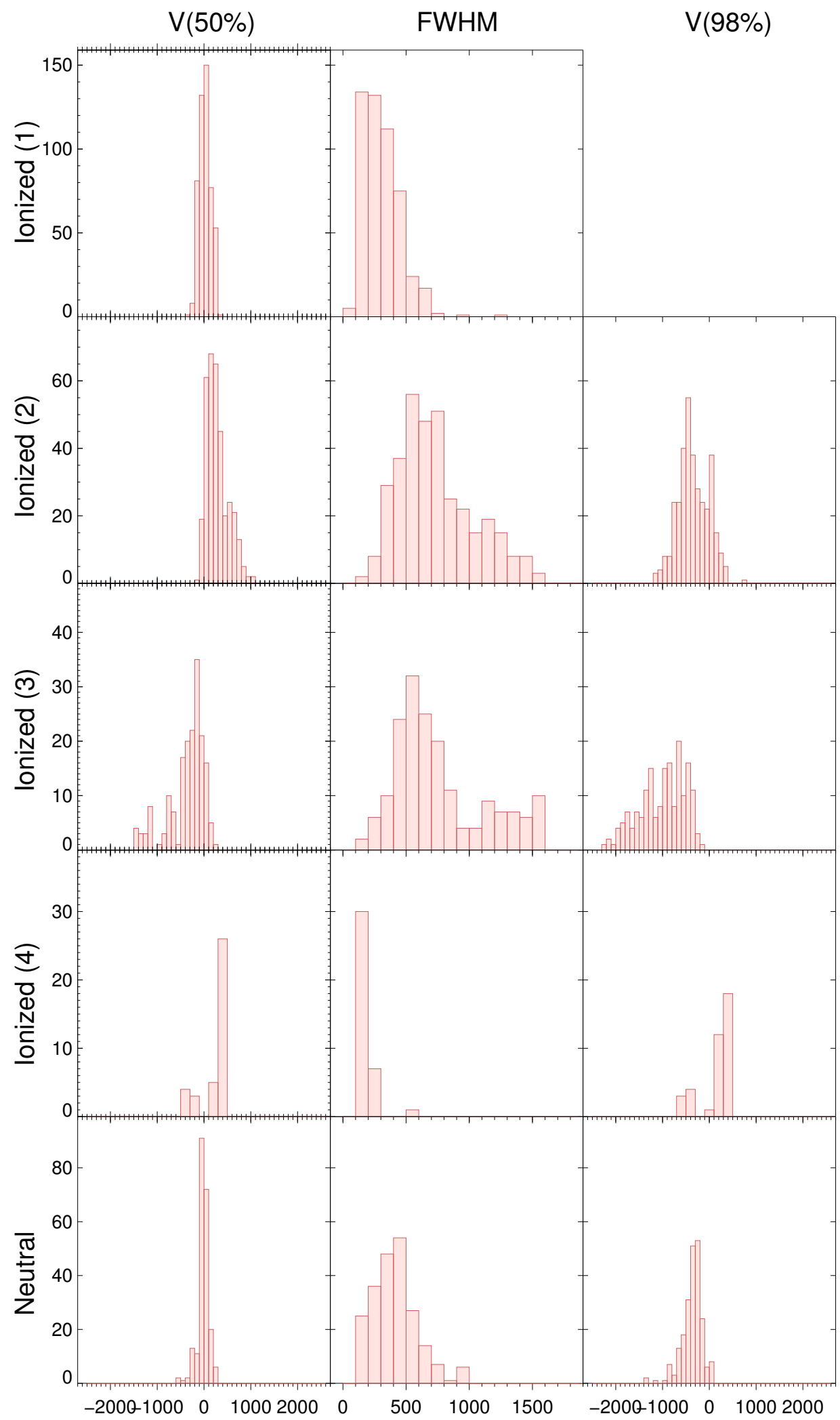

Figure 26. The same as Figure 5 but for Mrk 273. 


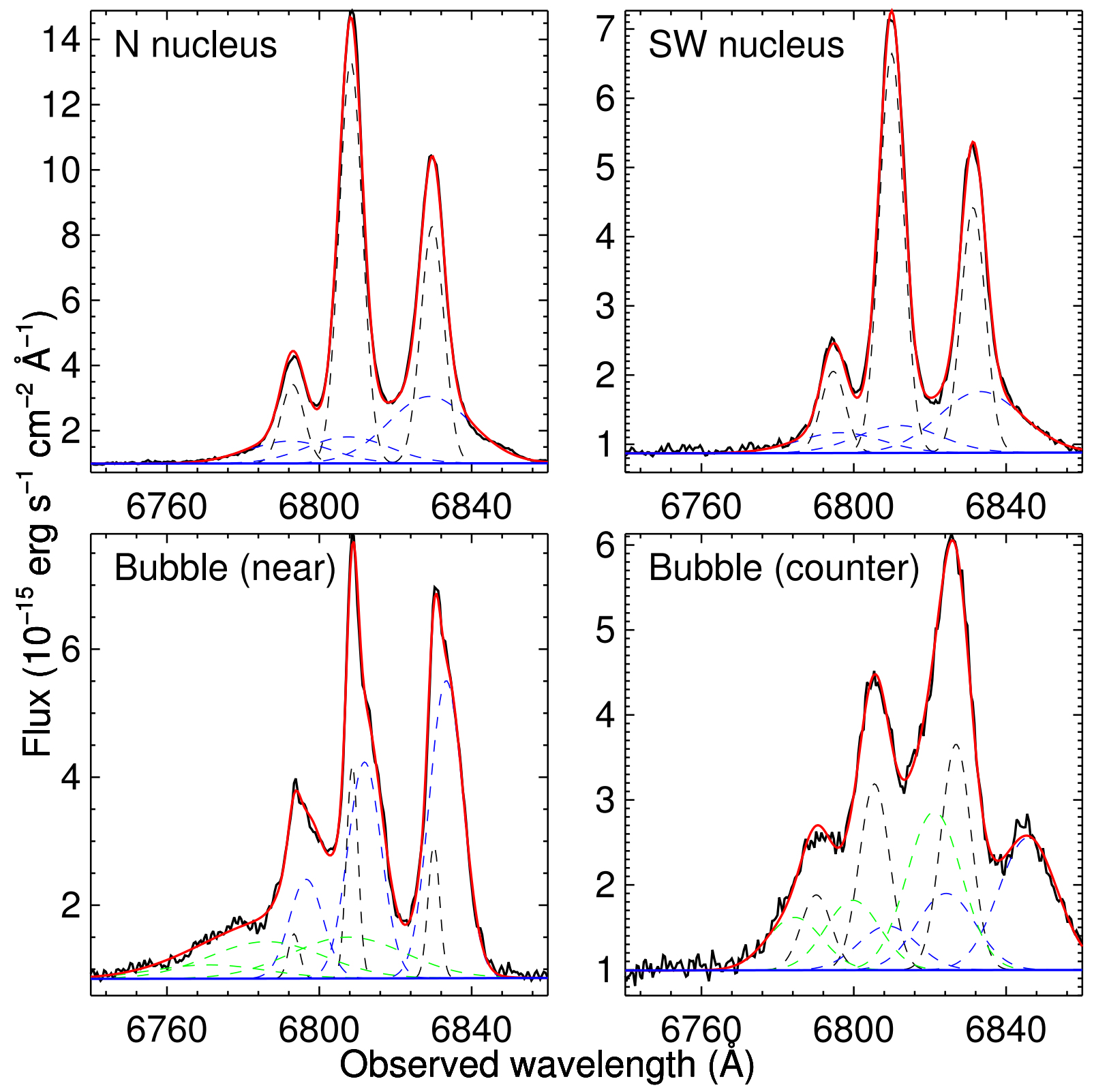

Figure 27. $\mathrm{H} \alpha+[\mathrm{N}$ II $] 6548,6583 \AA$ spectra of four spaxels in Mrk 273: nearest the N and SW nuclei, in the near-side superbubble, and in the counter-propagating superbubble. The solid black line is the data, the solid red line the total fit, the solid blue line the continuum fit, and the dashed lines fits to individual lines. The different colors of the dashed lines represent different velocity components. Note the three components in each superbubble: the near- (blueshifted) and far- (redshifted) sides of the bubble, as well as the rotating component. 


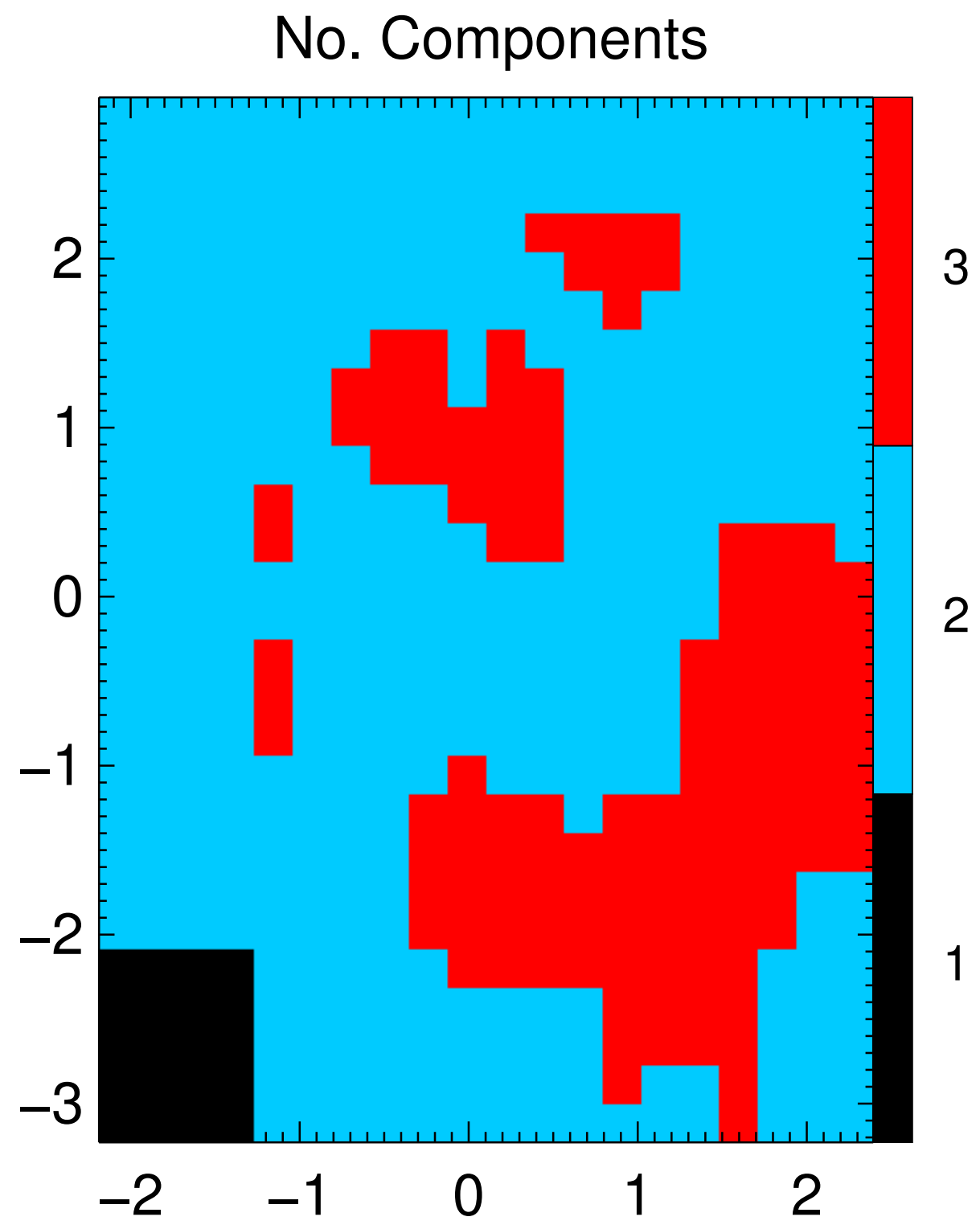

Figure 28. A color map of the number of emission-line components fit to each spaxel in Mrk 273. The axis labels are in kpc. 


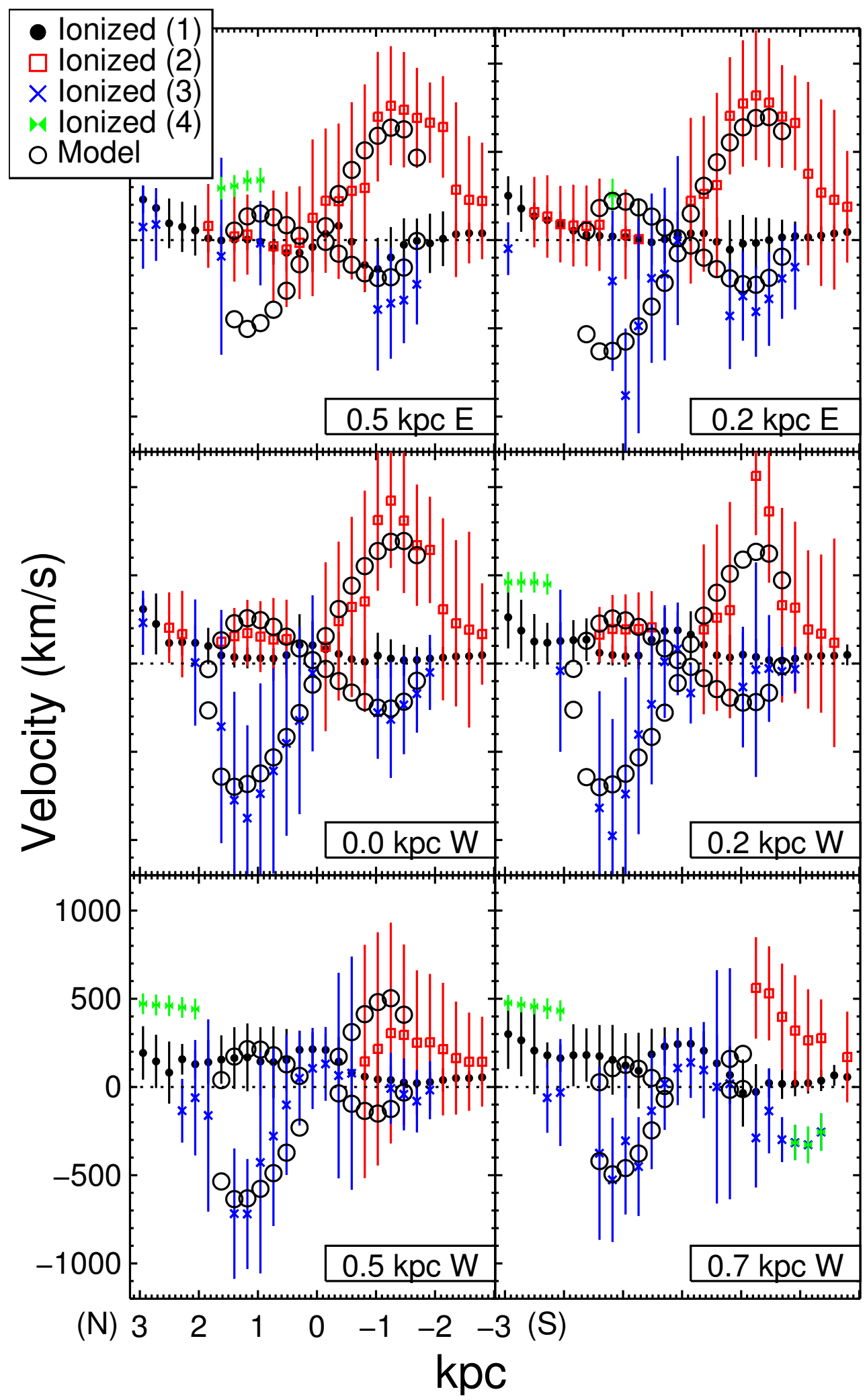

Figure 29. Position-velocity diagrams of the ionized gas in Mrk 273. Six columns are shown, with horizontal distance from the outflow center in kpc overlaid on each. Each ionized gas velocity component is shown $(1 /$ black solid circles = narrow, rotating; $2 / \mathrm{red}$ open squares $=$ broad, redshifted; 3 /blue crosses = broad, blueshifted; and 4/green bowties = narrow, non-rotating; $\delta 4.4 .2$, as well as the $n=2$ bipolar superbubble model fit to the data (open black circles). The vertical lines emerging from each point represent the spread in the velocity distribution $( \pm 1 \sigma)$. Model parameters are given in Table 5 (See $\$ \S 3.4 .2$ and 4.4 .3 for more details.) 


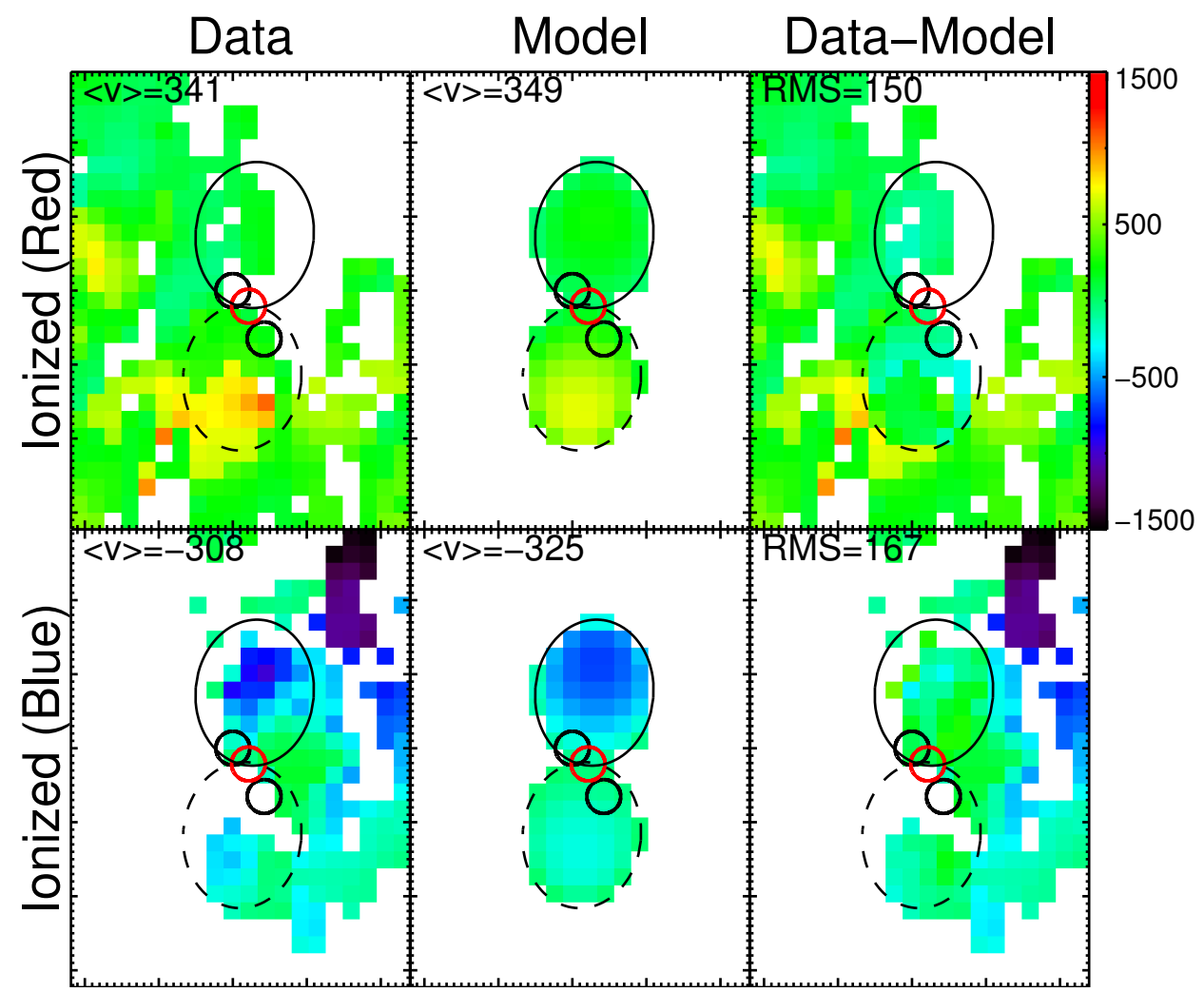

Figure 30. Color maps of superbubble model fits to the emission line velocity fields in Mrk 273. The fit shown is for $n=2$. The three columns show the data (left), model (middle), and their difference (right). In each panel, the solid line outlines the near-side bubble, and the dashed line the counter-bubble. Small black circles locate the two nuclei, while the red circle is the best-fit outflow center, assuming symmetry along the outflow axis. The numbers in the left and middle columns give the average velocity of that map within the model confines, while the numbers in the right panel are the RMS velocity differences within the same area. Model parameters are given in Table 5 (See $\$ \S 3.4 .2$ and 4.4 .3 for more details.) 
(a) F435W+F814W, 1'x1' (b) F435W+F814W, 15" $\times 15^{\prime \prime}$

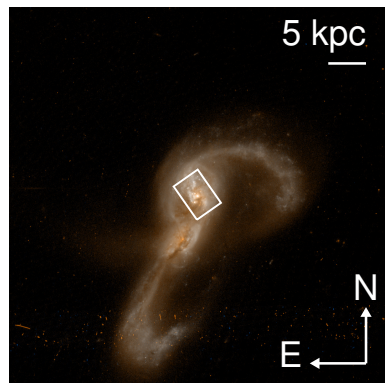

(e) $\log (F 435 W / F 814 W)$ $1^{\prime} \times 1^{\prime}$

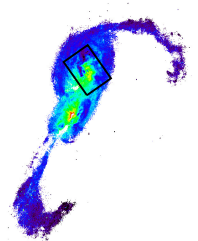

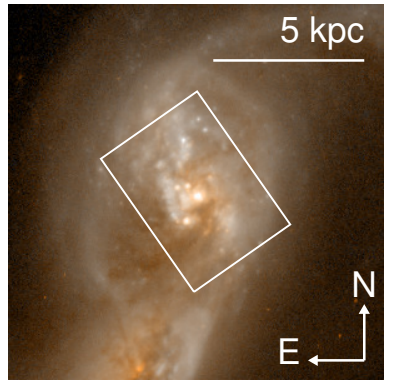

(f) $\log (\mathrm{F} 435 \mathrm{~W} / \mathrm{F} 814 \mathrm{~W})$

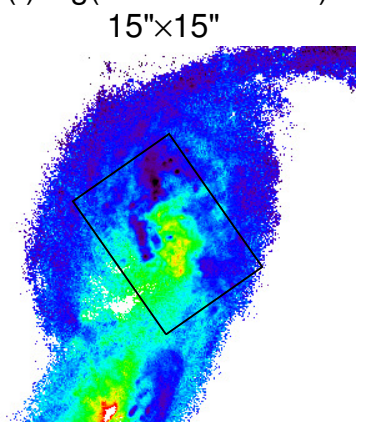

(c) $6100-6800 \AA$

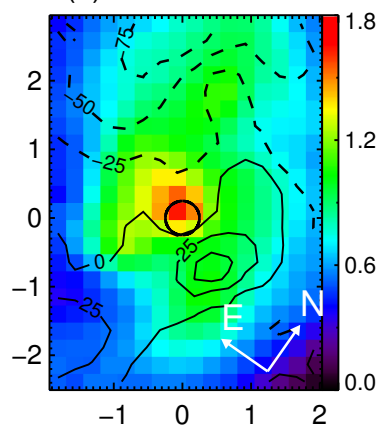

(g) $\log ^{-1}(\mathrm{~F} 435 \mathrm{~W} / \mathrm{F} 814 \mathrm{~W})$

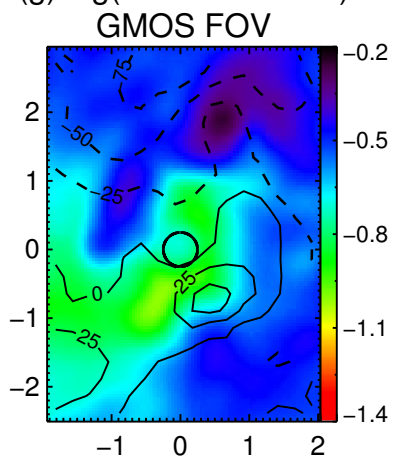

(d) $\mathrm{W}_{\mathrm{Na} D}(\AA)$

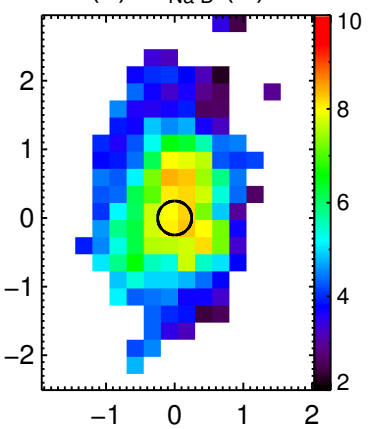

(h) $\mathrm{W}_{\mathrm{Na} D} / \delta \mathrm{W}_{\mathrm{Na} \text { D }}$

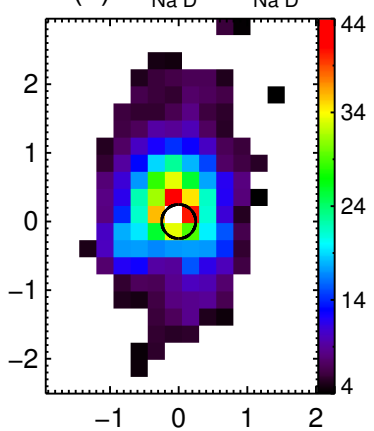

Figure 31. The same as Figure 1 but for VV 705:NW. Velocities are with respect to $z_{s y s}=0.04035$. 


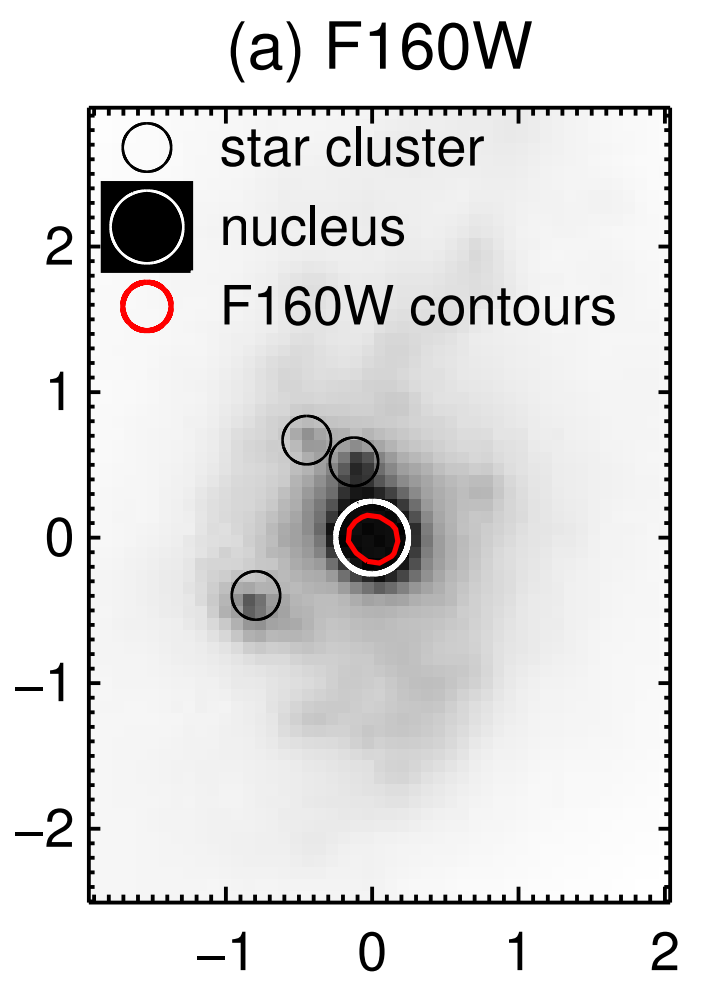

(c) F814W, smoothed

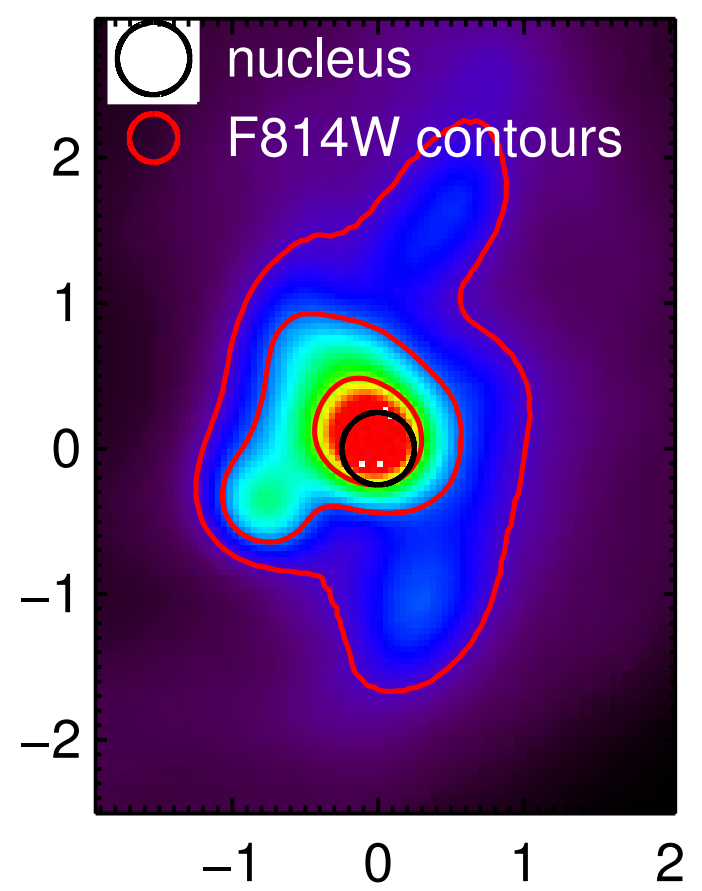

(b) F814W
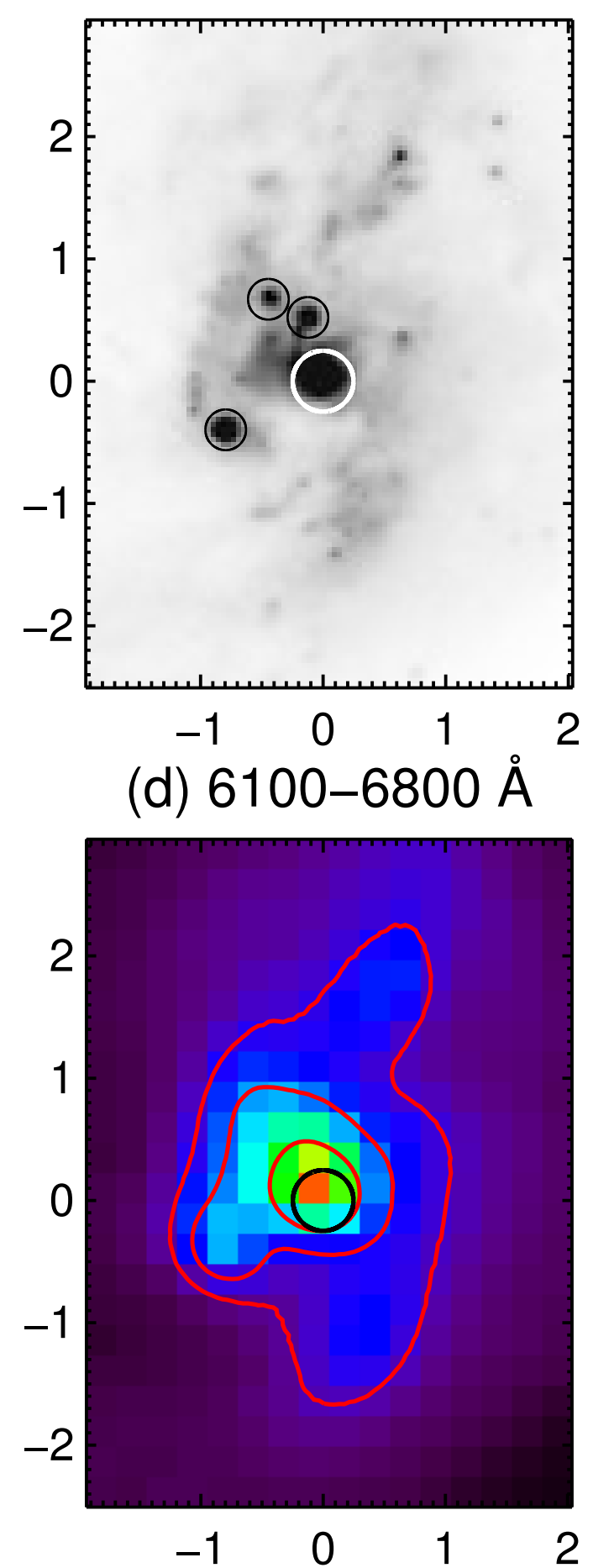

Figure 32. The same as Figure 2 but for VV 705:NW. 

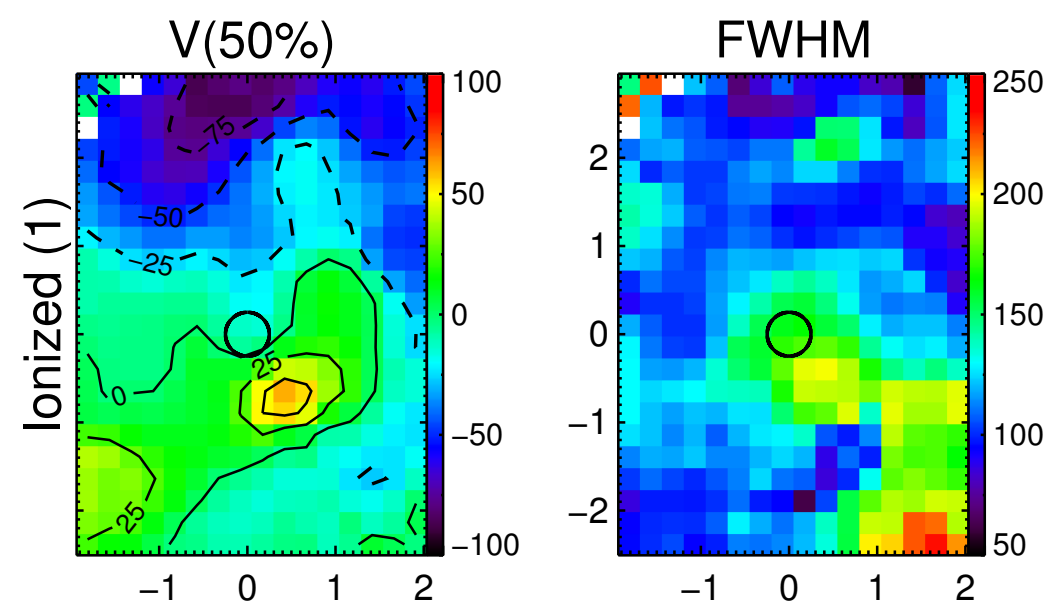

$\mathrm{V}(98 \%)$
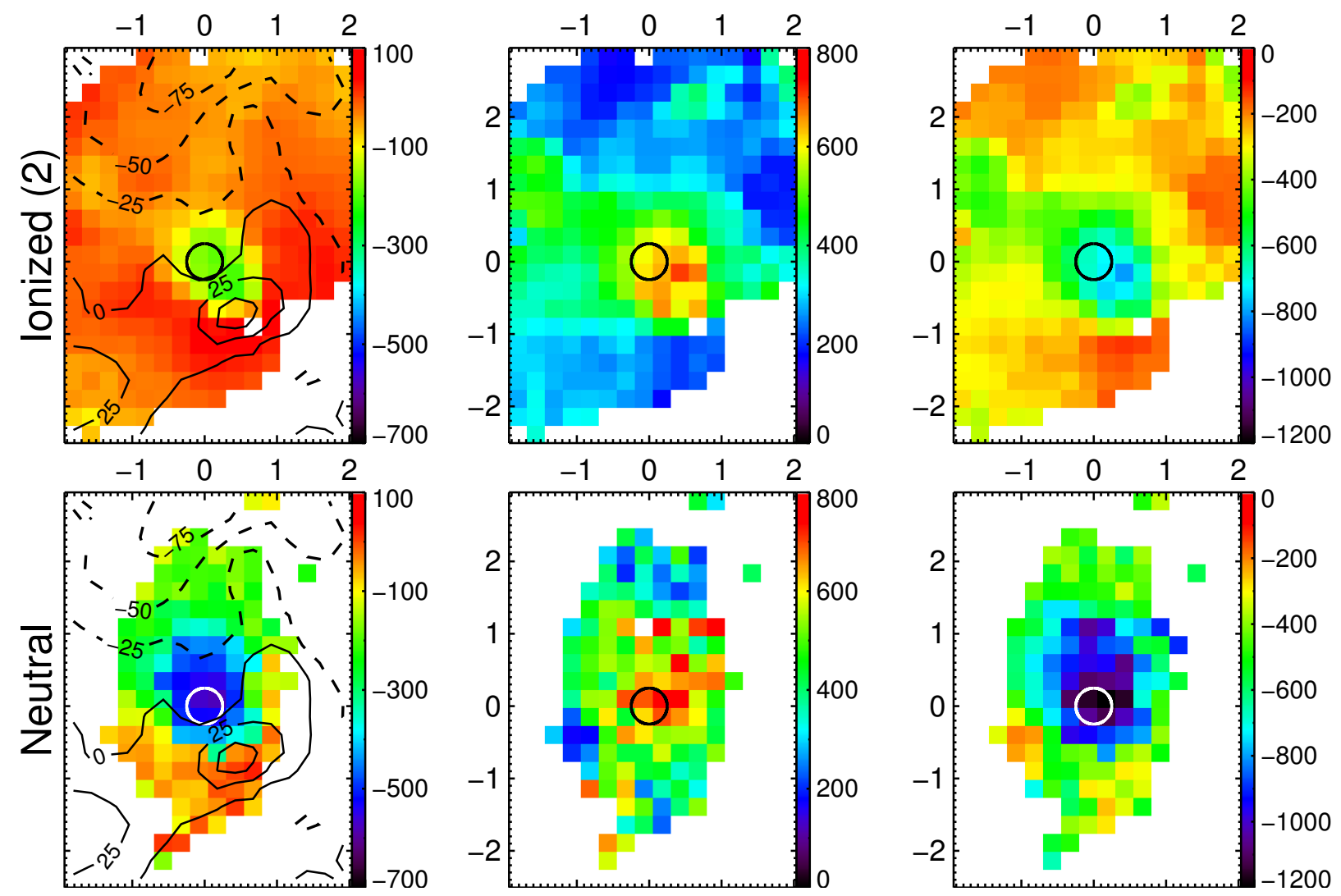

Figure 33. The same as Figure 3 but for VV 705:NW. 

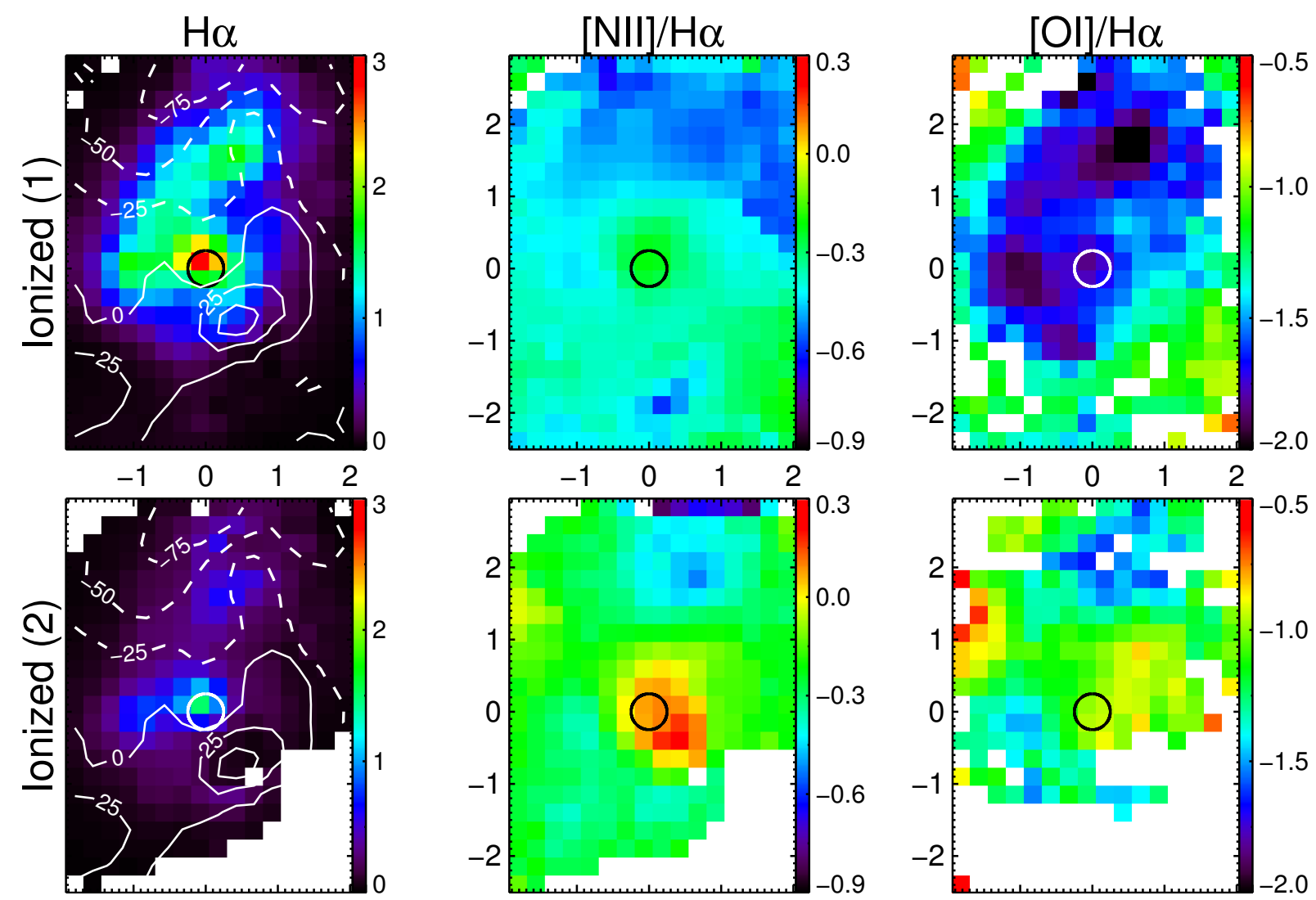

Figure 34. The same as Figure 4 but for VV 705:NW. 


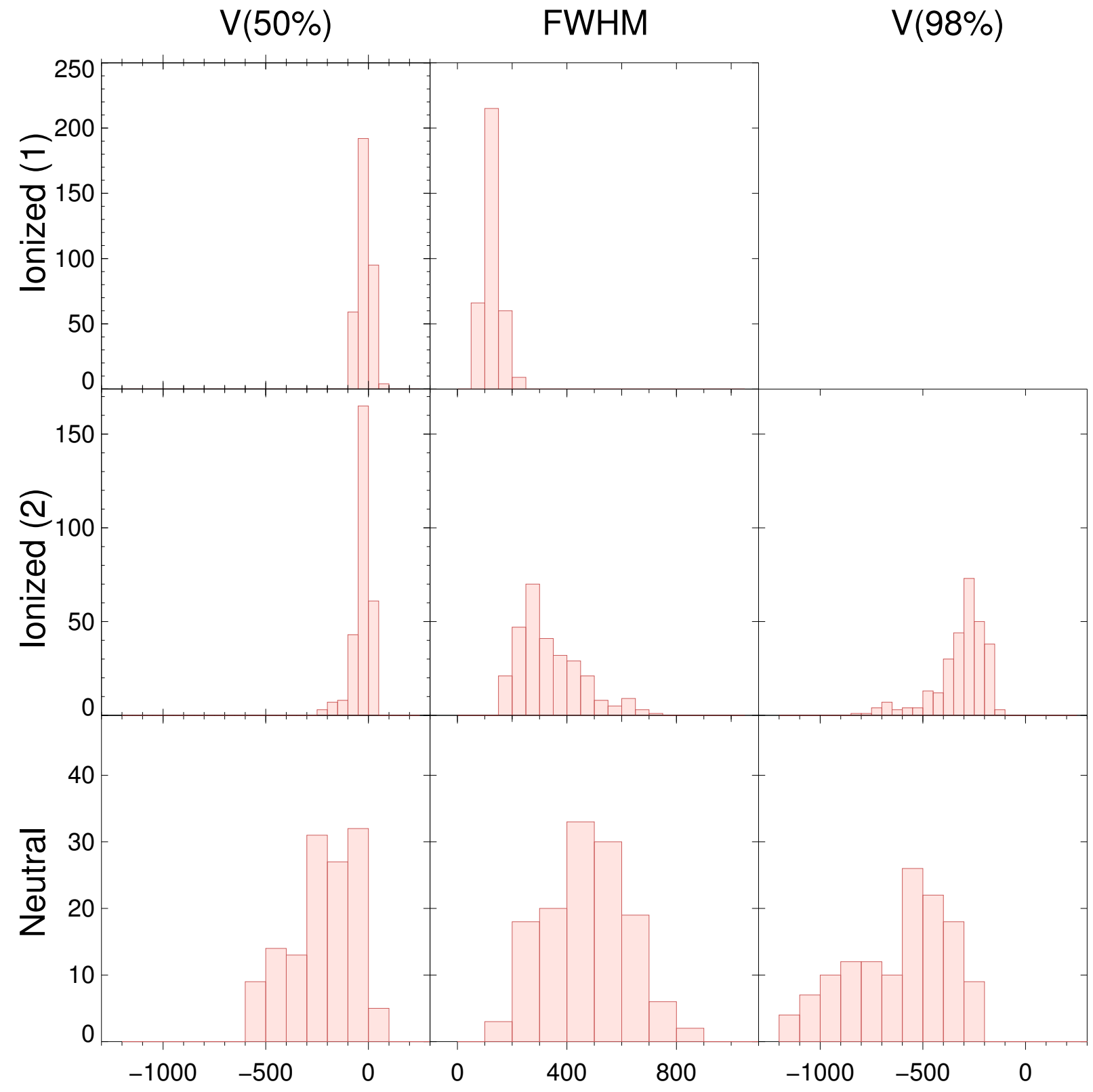

Figure 35. The same as Figure 5 but for VV 705:NW. 
(a) F435W+F814W, $1^{\prime} \times 1^{\prime}$

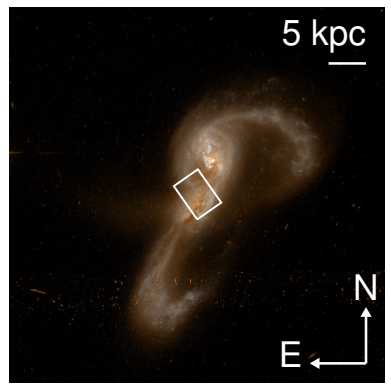

(e) $\log (\mathrm{F} 435 \mathrm{~W} / \mathrm{F} 814 \mathrm{~W})$

$1^{\prime} \times 1^{\prime}$

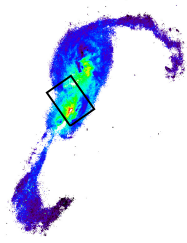

(b) $\mathrm{F} 435 \mathrm{~W}+\mathrm{F} 814 \mathrm{~W}, 15 " \times 15 "$

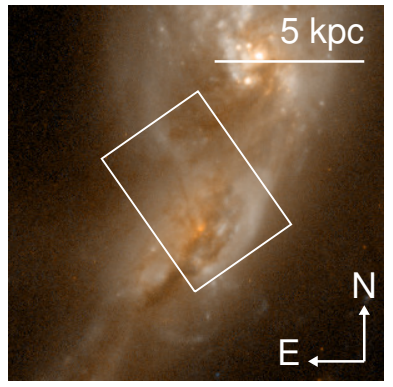

(f) $\log (F 435 W / F 814 W)$

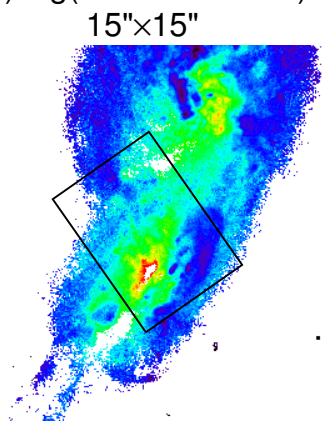

(c) $6100-6800 \AA$

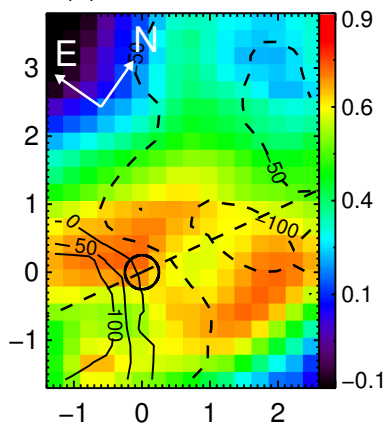

(g) $\log (\mathrm{F} 435 \mathrm{~W} / \mathrm{F} 814 \mathrm{~W})$

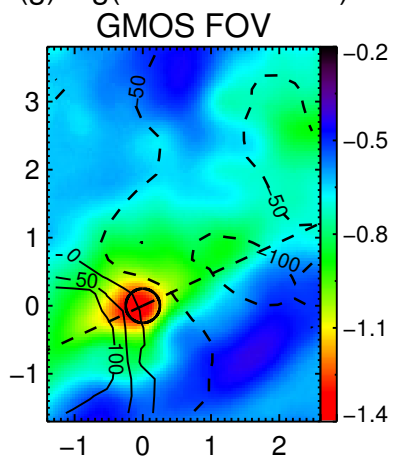

(d) $\mathrm{W}_{\mathrm{Na} D}(\AA)$

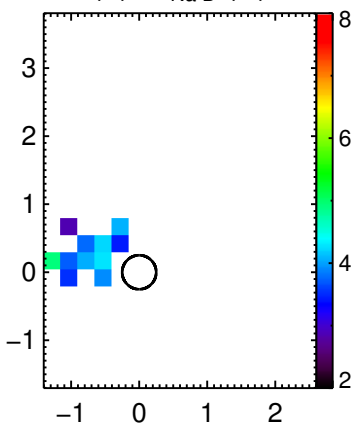

(h) $\mathrm{W}_{\mathrm{Na} \text { D }} / \delta \mathrm{W}_{\mathrm{Na}} \mathrm{D}$

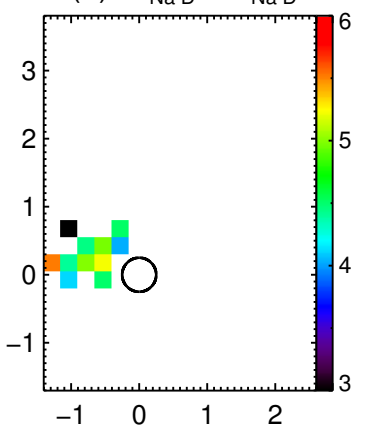

Figure 36. The same as Figure 1 but for VV 705:SE. Velocities are with respect to $z_{s y s}=0.0407$. The dashed line is the estimated line of nodes based on the current dataset $\left(\mathrm{PA}=-30^{\circ}\right)$. The nuclear position is based on the $I$-band peak. 
(a) F160W

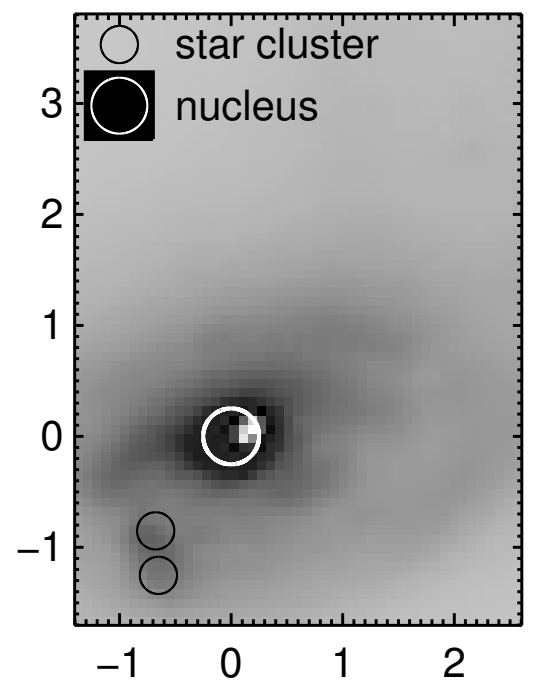

(c) F814W, smoothed

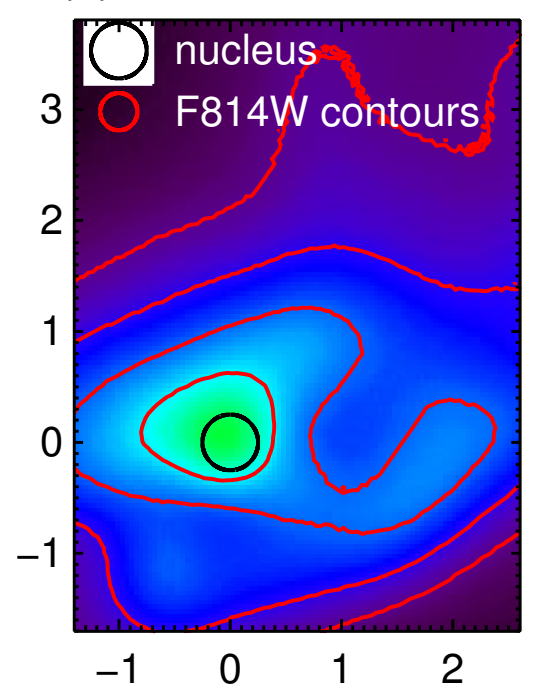

(b) F814W
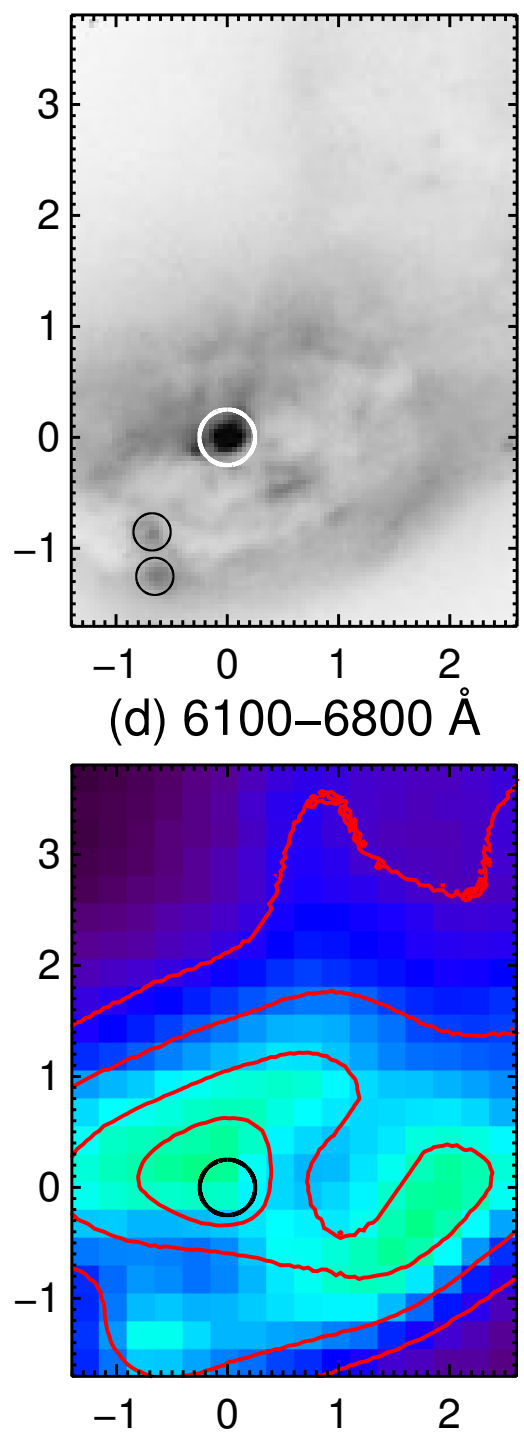

Figure 37. The same as Figure 2 but for VV 705:SE. Note that saturated or bad pixels are present near the nucleus in the F160W image, and that the star clusters do not appear in the F160W image; as a result, the F814W image is used to locate the nucleus. 

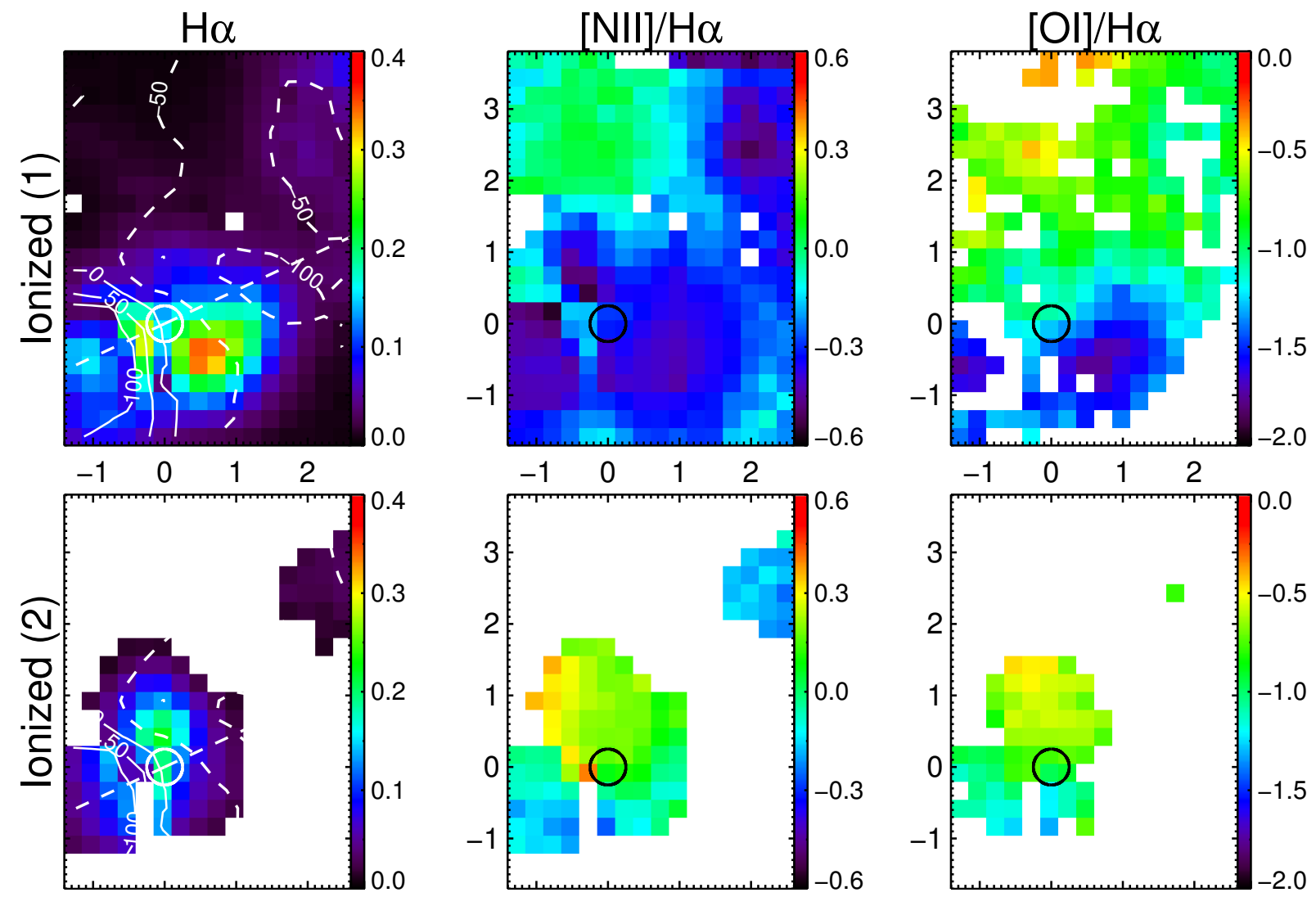

Figure 38. The same as Figure 3 but for VV 705:SE. 

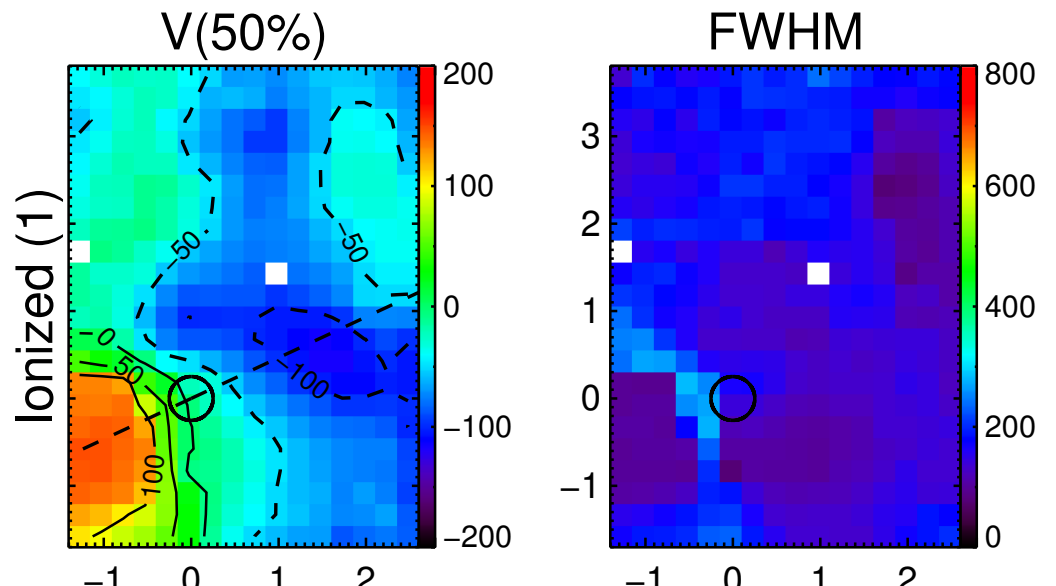

$\mathrm{V}(98 \%)$
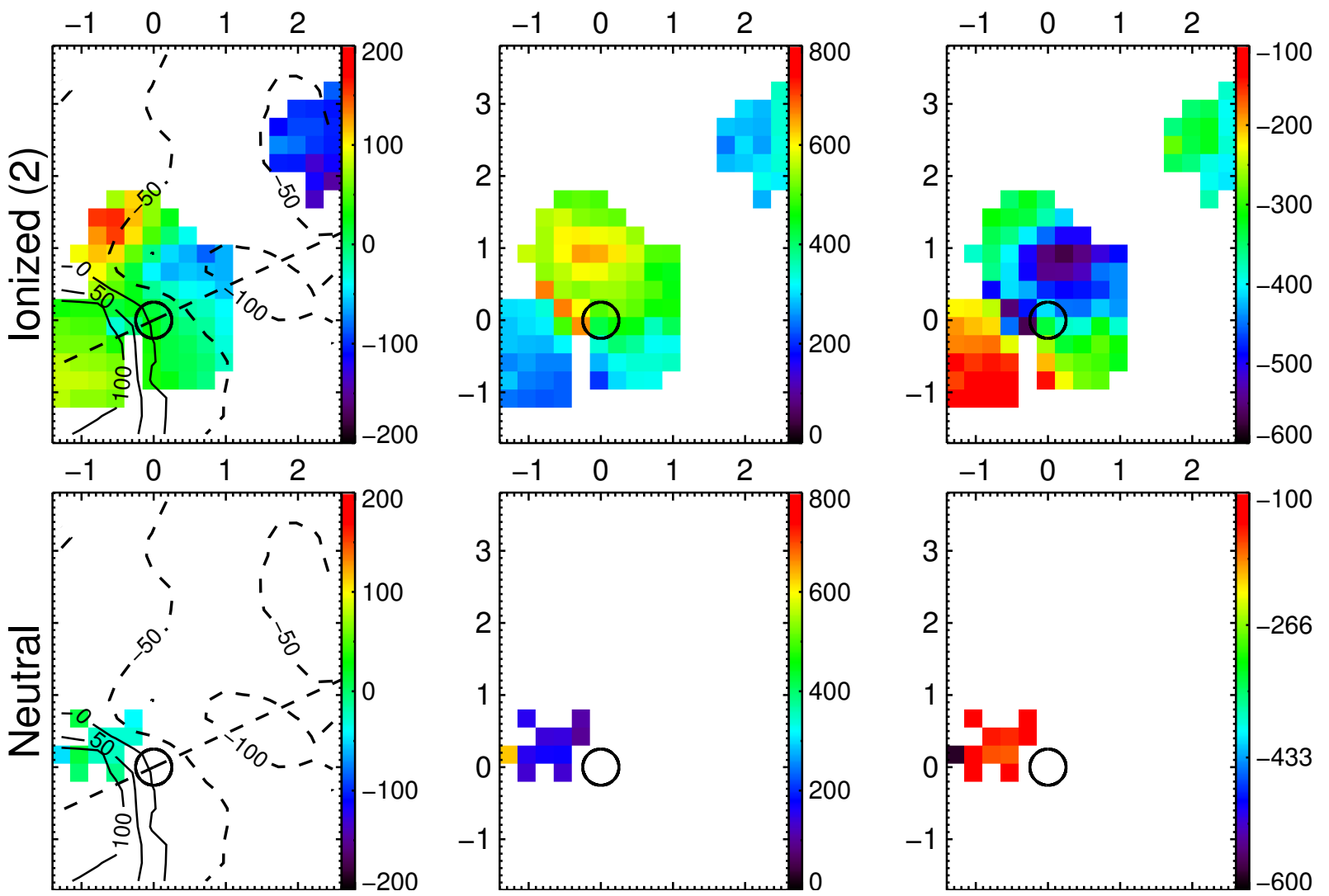

Figure 39. The same as Figure 4 but for VV 705:SE. 


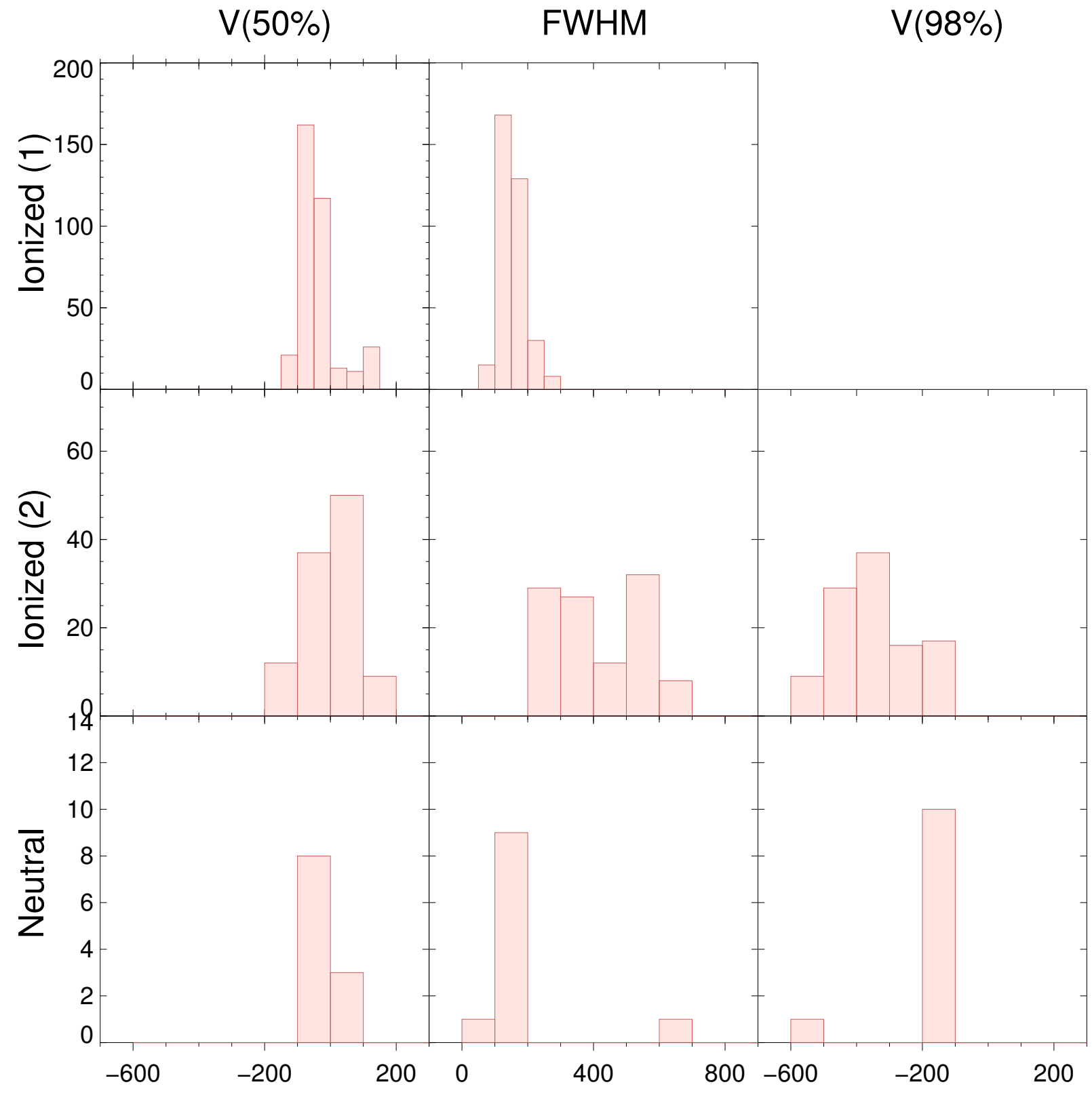

Figure 40. The same as Figure 5 but for VV 705:SE. 


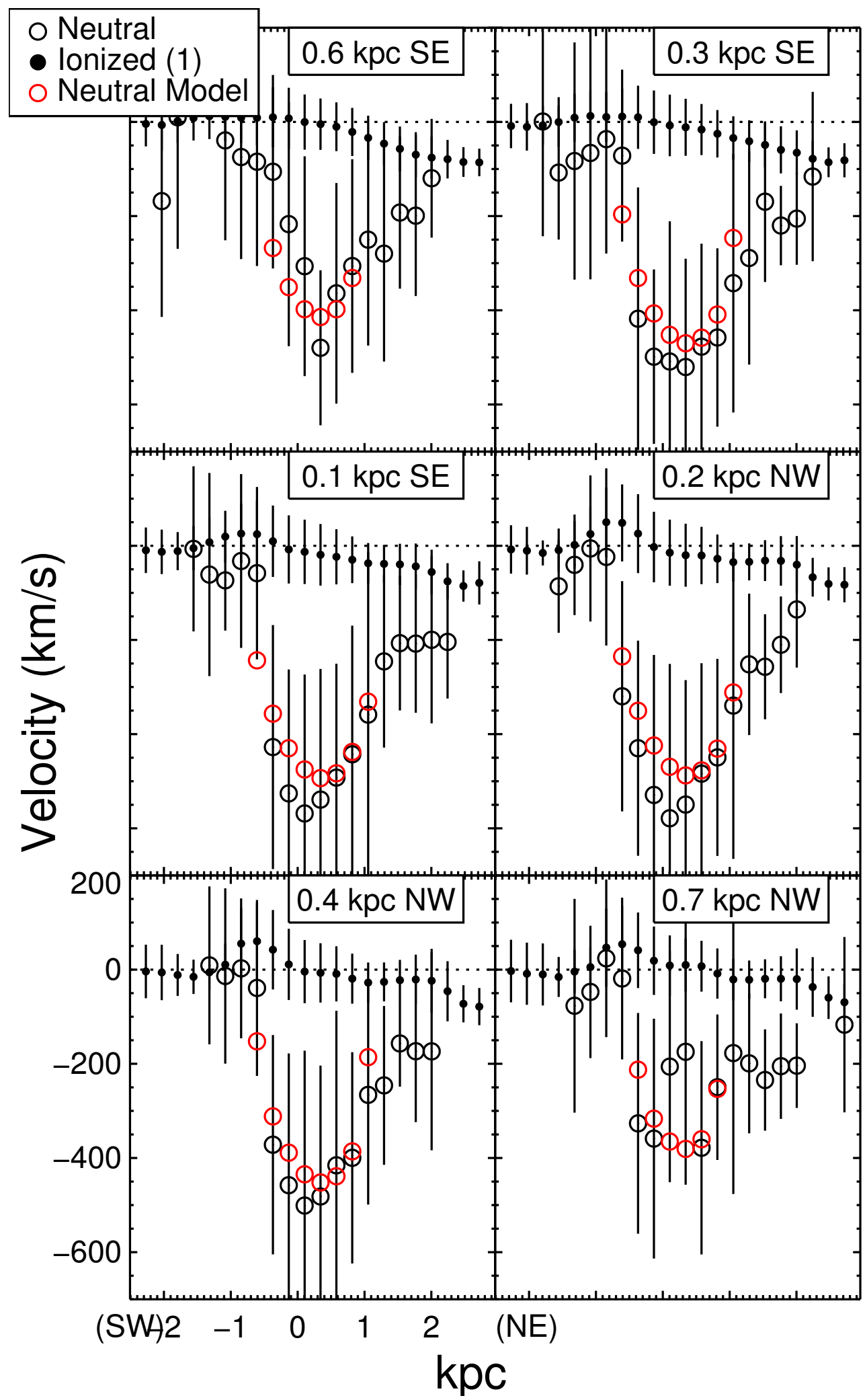

Figure 41. Position-velocity diagrams of the neutral gas in VV 705:NW. Six columns are shown, with horizontal distance from the outflow center in kpc overlaid on each. The neutral gas $v_{50 \%}$ values are shown (black open circles), as well as the $n=2$ bipolar superbubble model fit to the data (red open circles) and the narrow ionized gas component (small black points). The vertical lines emerging from each point represent the spread in the velocity distribution $( \pm 1 \sigma)$. Model parameters are given in Table 5 (See $\S 4.5 .2$ for more details.) 


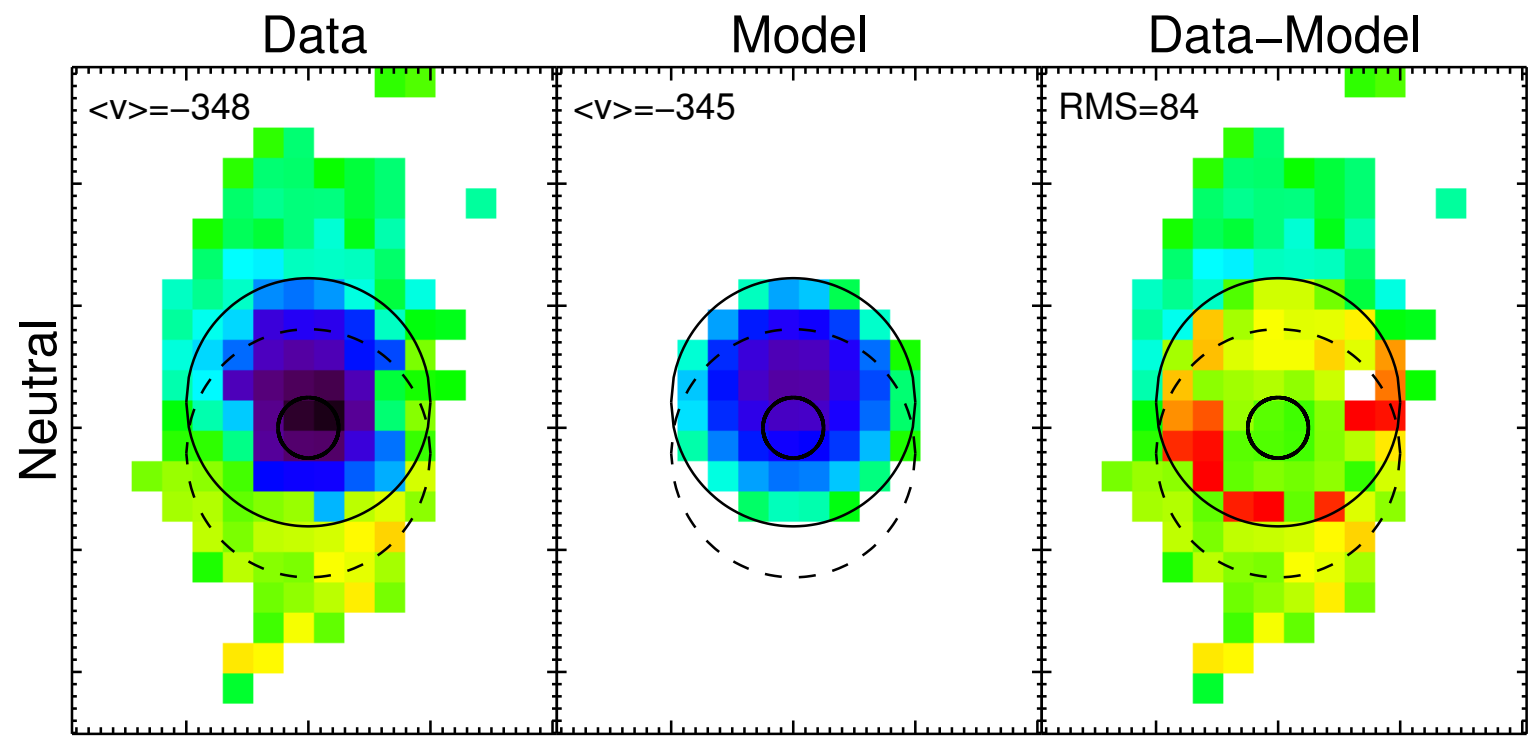

Figure 42. Color maps of superbubble model fits to the absorption line velocity field in VV 705:NW. The fit shown is for $n=2$. The three columns show the data (left), model (middle), and their difference (right). In each panel, the solid line outlines the near-side bubble, the dashed lines the expected counter-bubble (the counter-bubble is hidden behind the disk). A small black circle locates the nucleus and outflow center. The numbers in the left and middle columns give the average velocity of that map within the model confines, while the number in the right panel is the RMS velocity difference within the same area. Model parameters are given in Table 5 (See $\delta \S 3.4 .2$ and 4.5 .2 for more details.) 
(a) $\mathrm{F} 435 \mathrm{~W}+\mathrm{F} 814 \mathrm{~W}, 1^{\prime} \times 1^{\prime}$

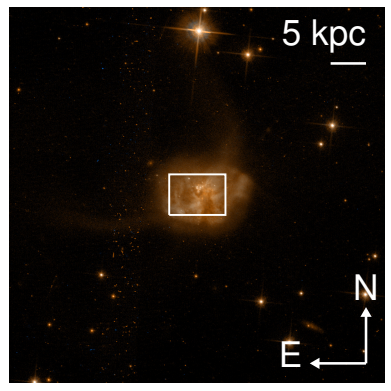

(e) $\log (F 435 W / F 814 W)$

$1^{\prime} \times 1^{\prime}$

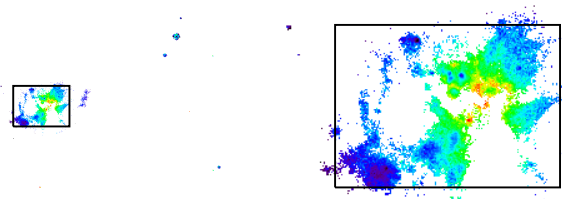
$15 " \times 15 "$ (c) $6100-6800 \AA$

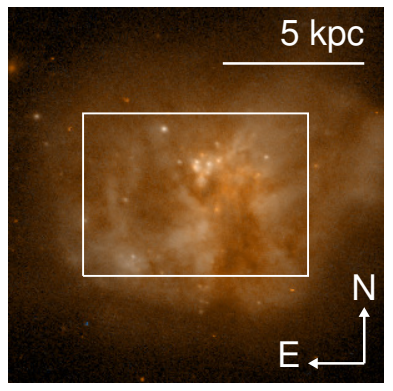

(f) $\log (F 435 W / F 814 W)$

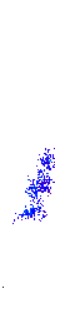

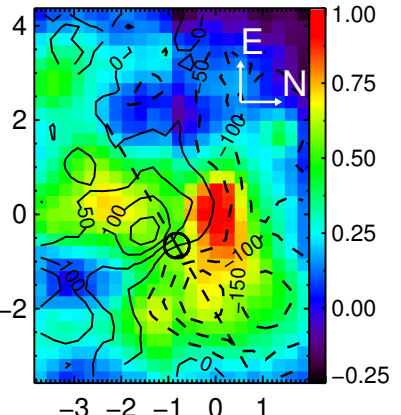

$-3-2-1 \stackrel{0}{1}$
$\log (F 435 W / F 814 W)$

GMOS FOV

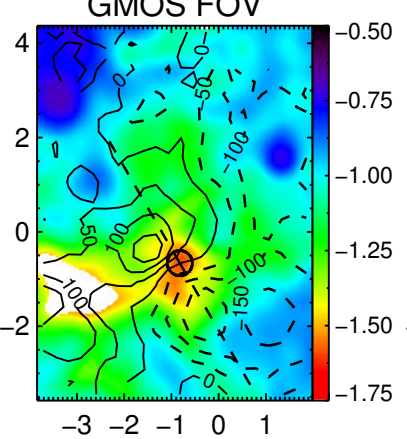

(d) $\mathrm{W}_{\mathrm{Na} \text { D }}(\AA \AA)$

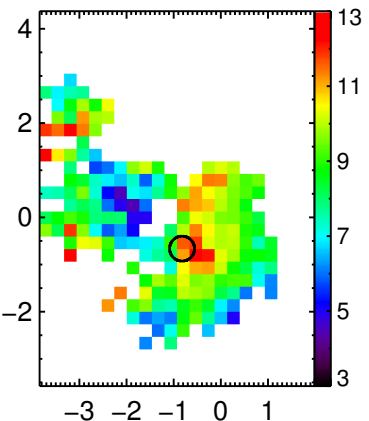

(h) $\mathrm{W}_{\mathrm{NaD}} / \delta \mathrm{W}_{\mathrm{NaD}}$

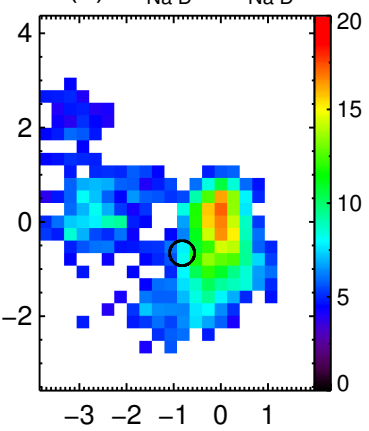

Figure 43. The same as Figure 1, but for F17207-0014. Velocities are with respect to $z_{s y s}=0.0430$, and the dashed line represents the position angle of the CO disk (Downes \& Solomon 1998). 
(a) F160W

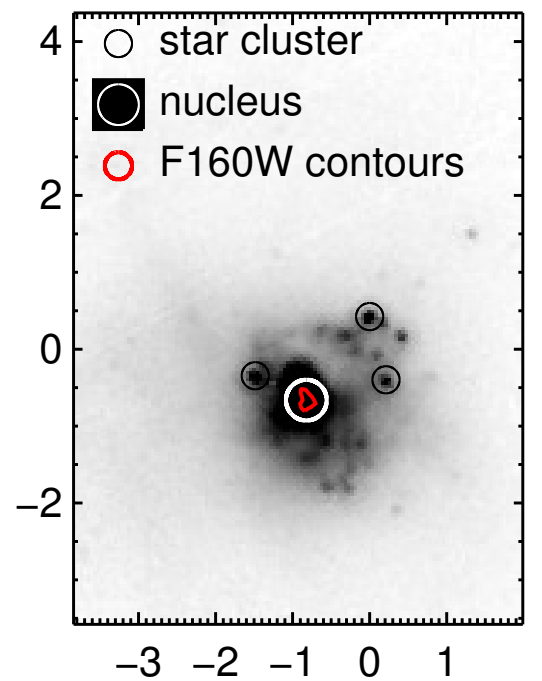

(c) F814W, smoothed

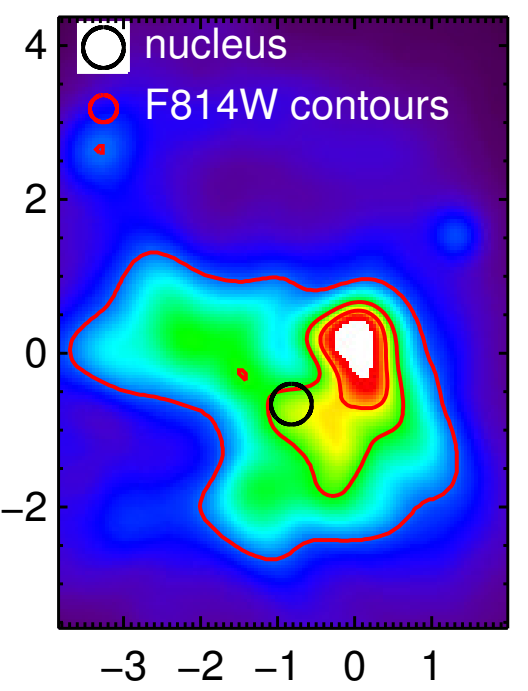

(b) F814W
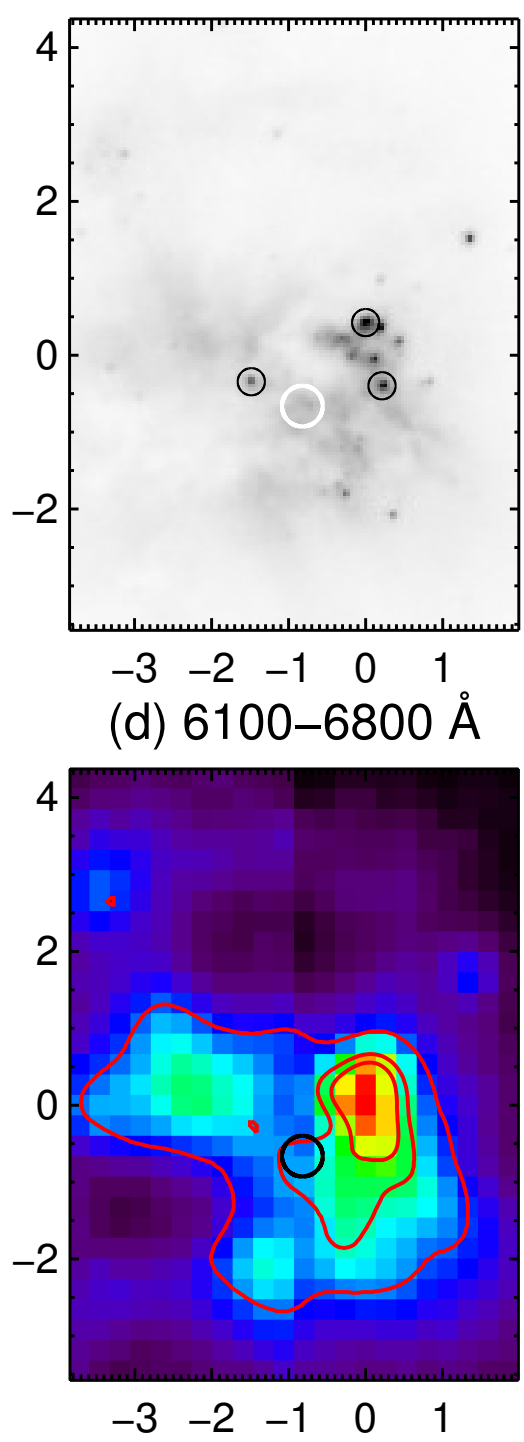

Figure 44. The same as Figure 2 but for F17207-0014. 

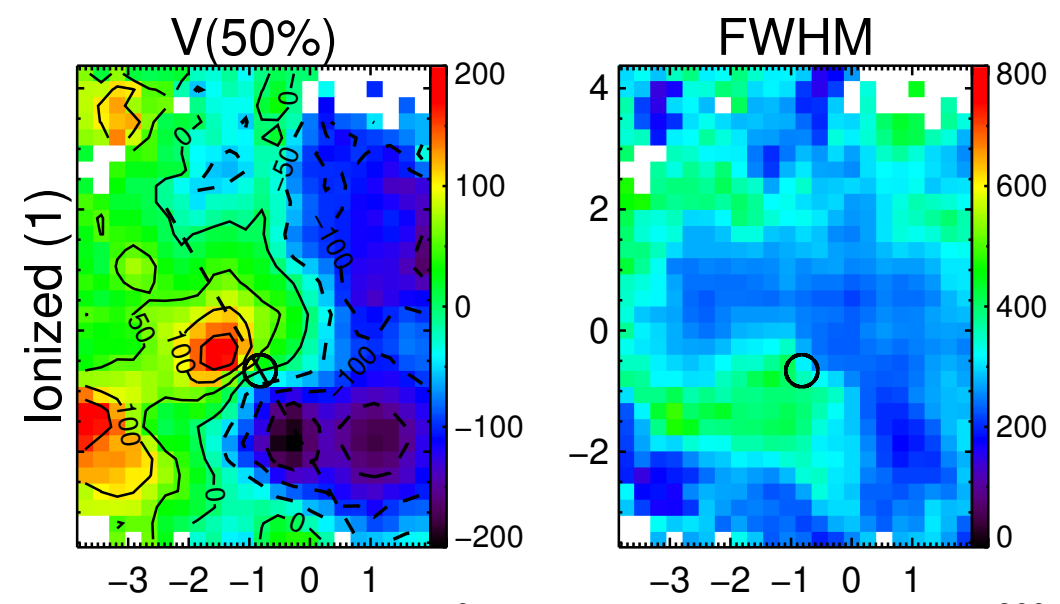

$\mathrm{V}(98 \%)$
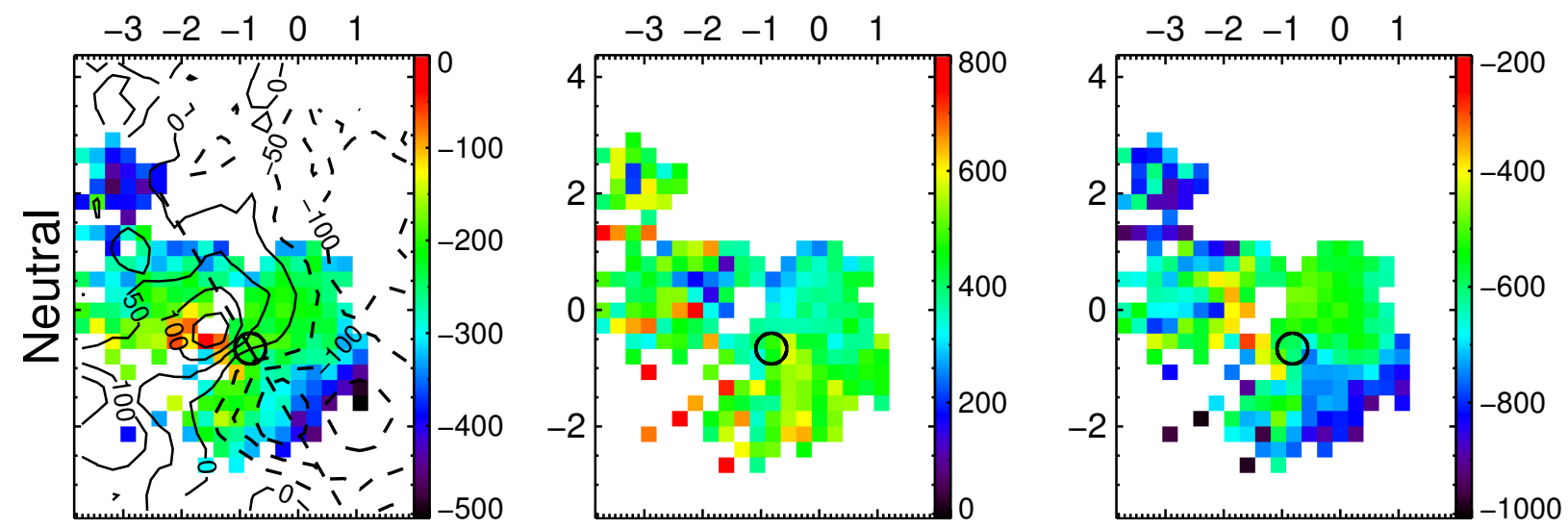

Figure 45. The same as Figure 3 but for F17207-0014.
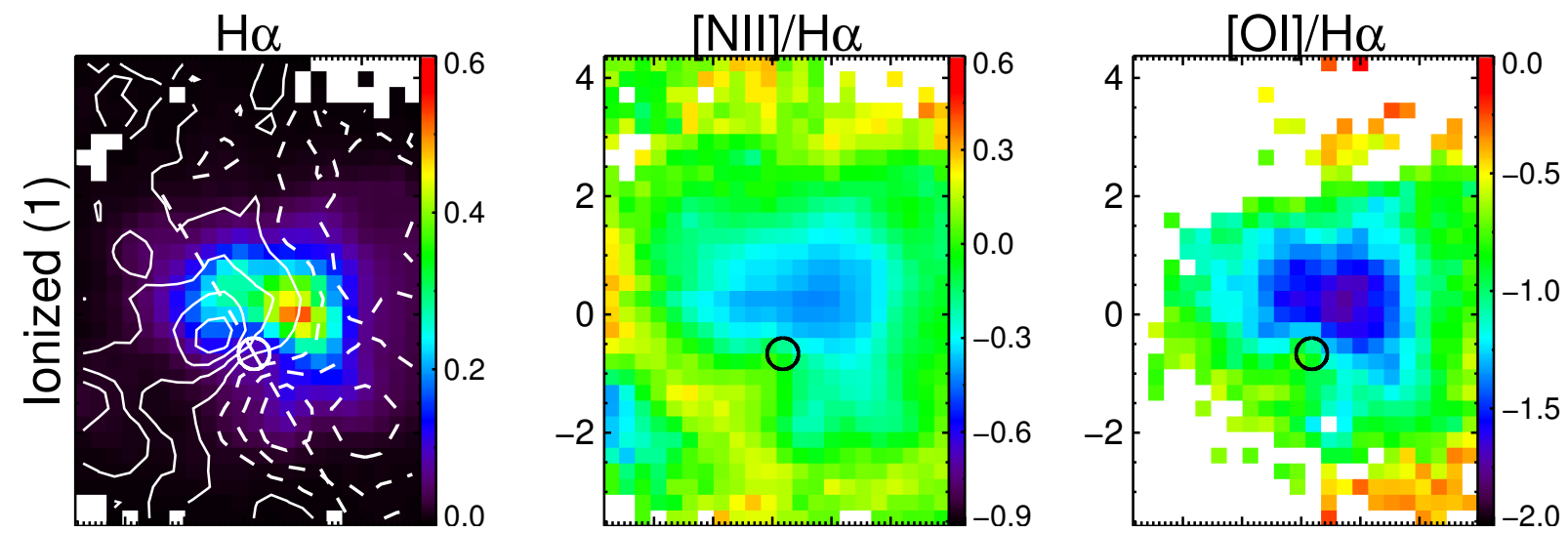

Figure 46. The same as Figure 4 but for F17207-0014. 


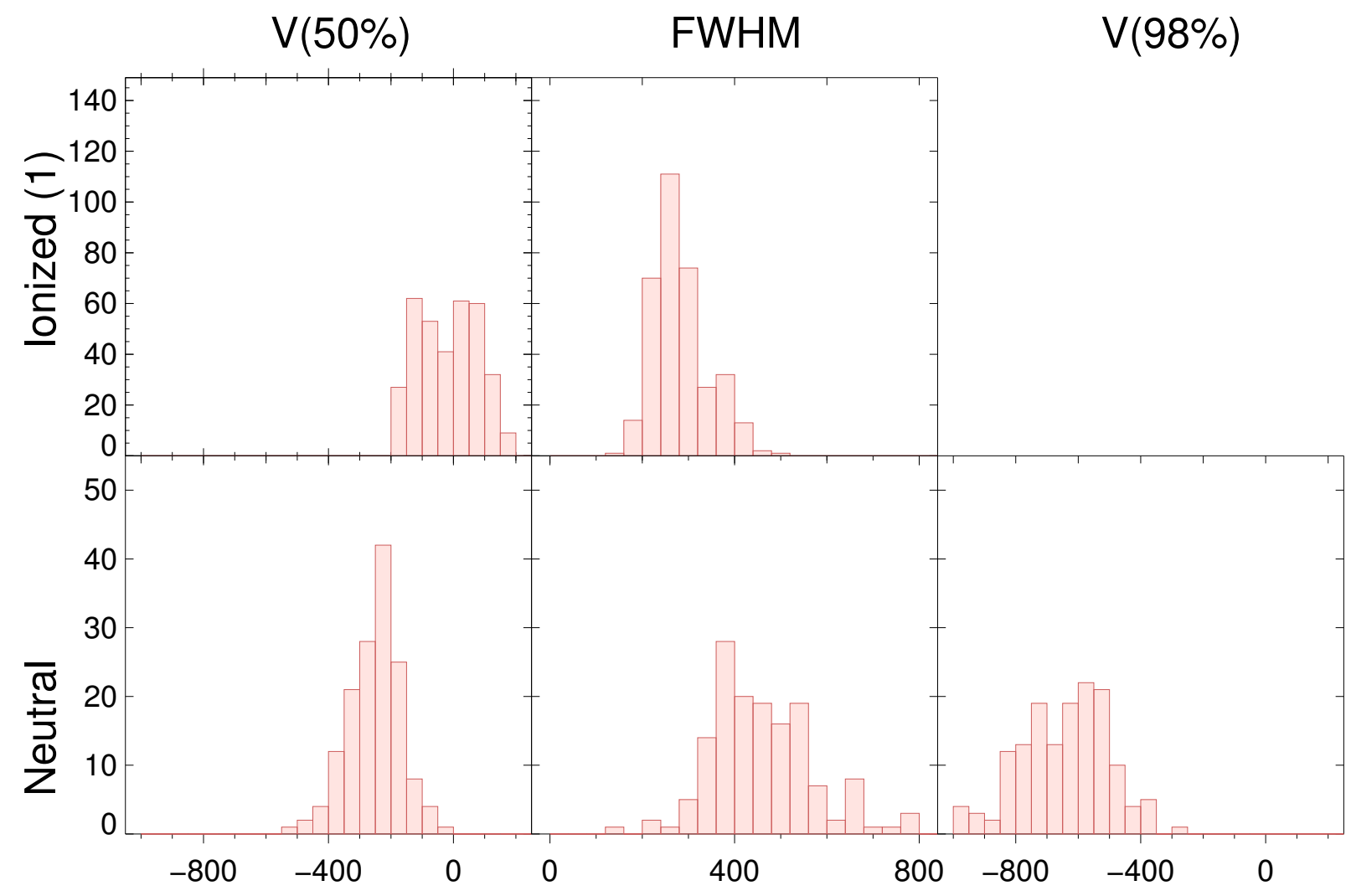

Figure 47. The same as Figure 5 but for F17207-0014.

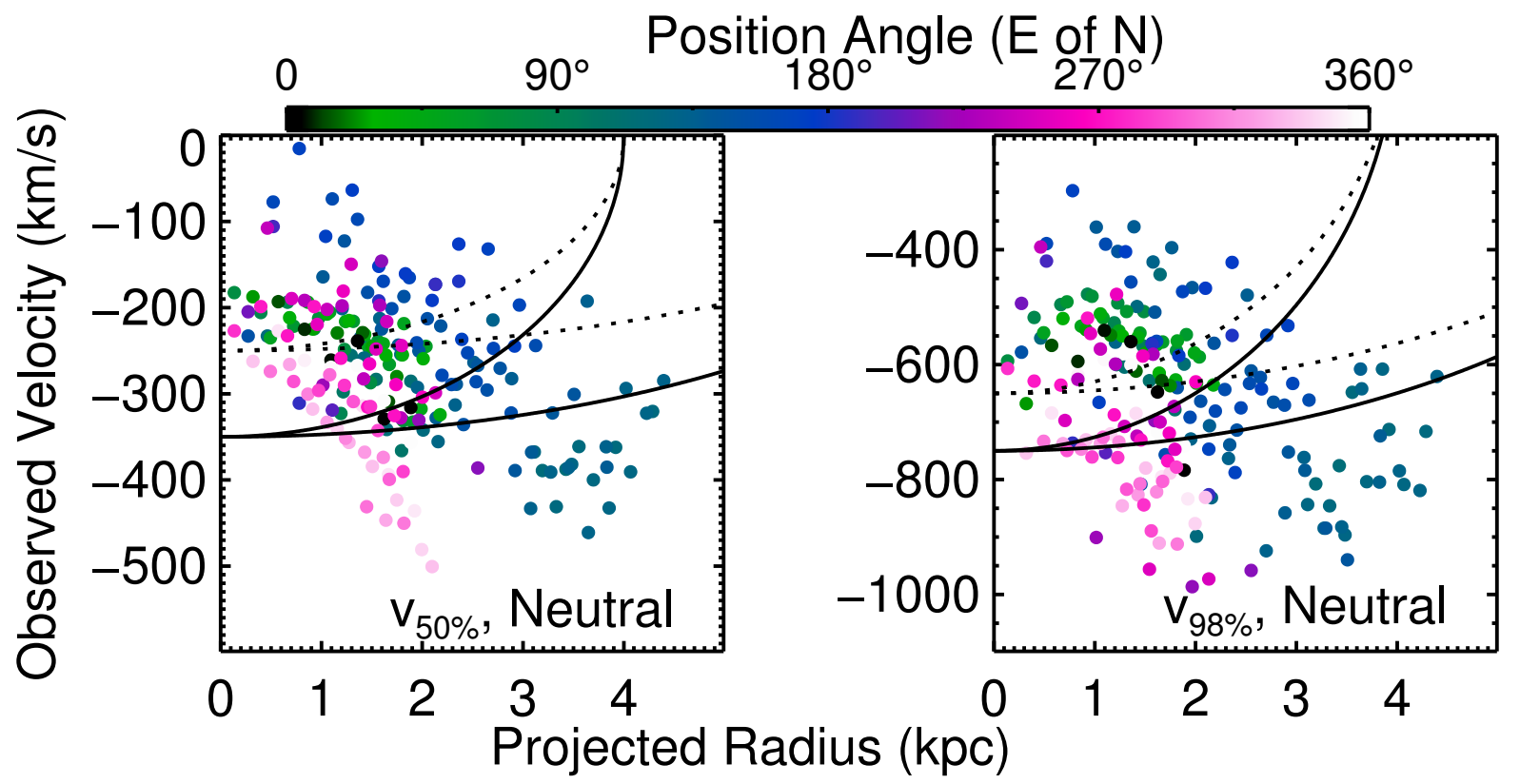

Figure 48. Observed velocity $\left(v_{50 \%}\right.$ and $\left.v_{98 \%}\right)$ vs. projected galactocentric radius in the outflowing neutral gas of F17207-0014. Each point represents one spaxel, and the position angle ( $\mathrm{E}$ of $\mathrm{N}$ ) of the spaxel with respect to the outflow center is represented by its color. The lines represent single radius free wind models $(\S 3$ at constant velocity for $R=4 \mathrm{kpc}$ and $8 \mathrm{kpc}$ (the velocity for a given model is given by the value at $R_{\text {proj }}=0 \mathrm{kpc}$ ). The observed increase of velocity with projected galactocentric radius is inconsistent with the SRFW model. 


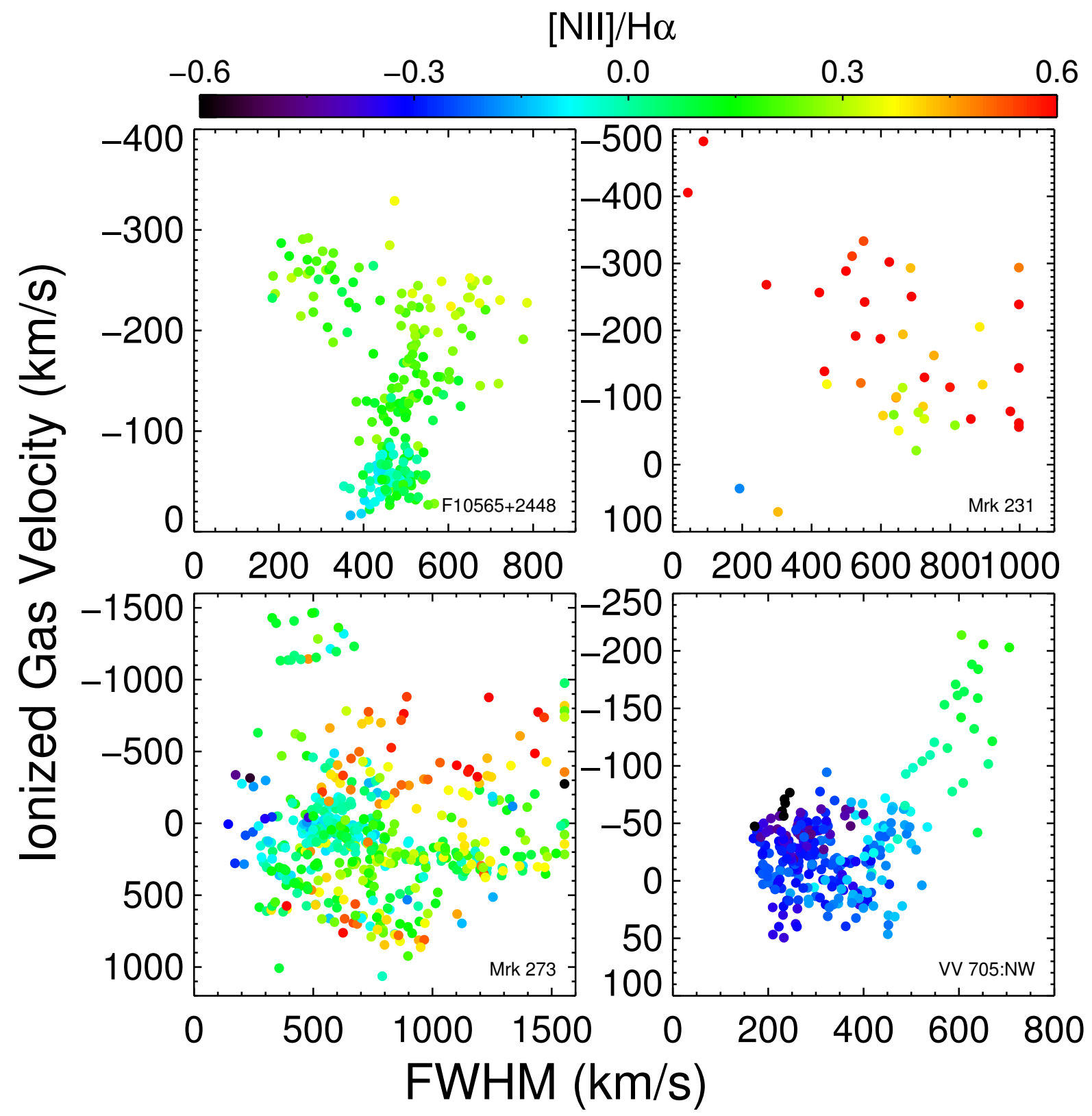

Figure 49. Ionized gas velocity $\left(v_{50 \%}\right)$ vs. FWHM for the broad emission line component, in the four nuclei in our sample with ionized gas outflows and resolved $[\mathrm{N} \mathrm{II}] / \mathrm{H} \alpha$ data. The color of each point represents its $[\mathrm{N} \mathrm{II}] / \mathrm{H} \alpha$ flux ratio. Within a given source, there is a clear trend for spaxels of higher velocity with respect to systemic and higher FWHM to have higher gas excitation. This suggests that the wind is being shock-excited, though there may be a contribution from AGN photoionization in those systems where an AGN is present, as well as a contribution from stellar photoionization at the lowest velocities $(\S 5.2$. 


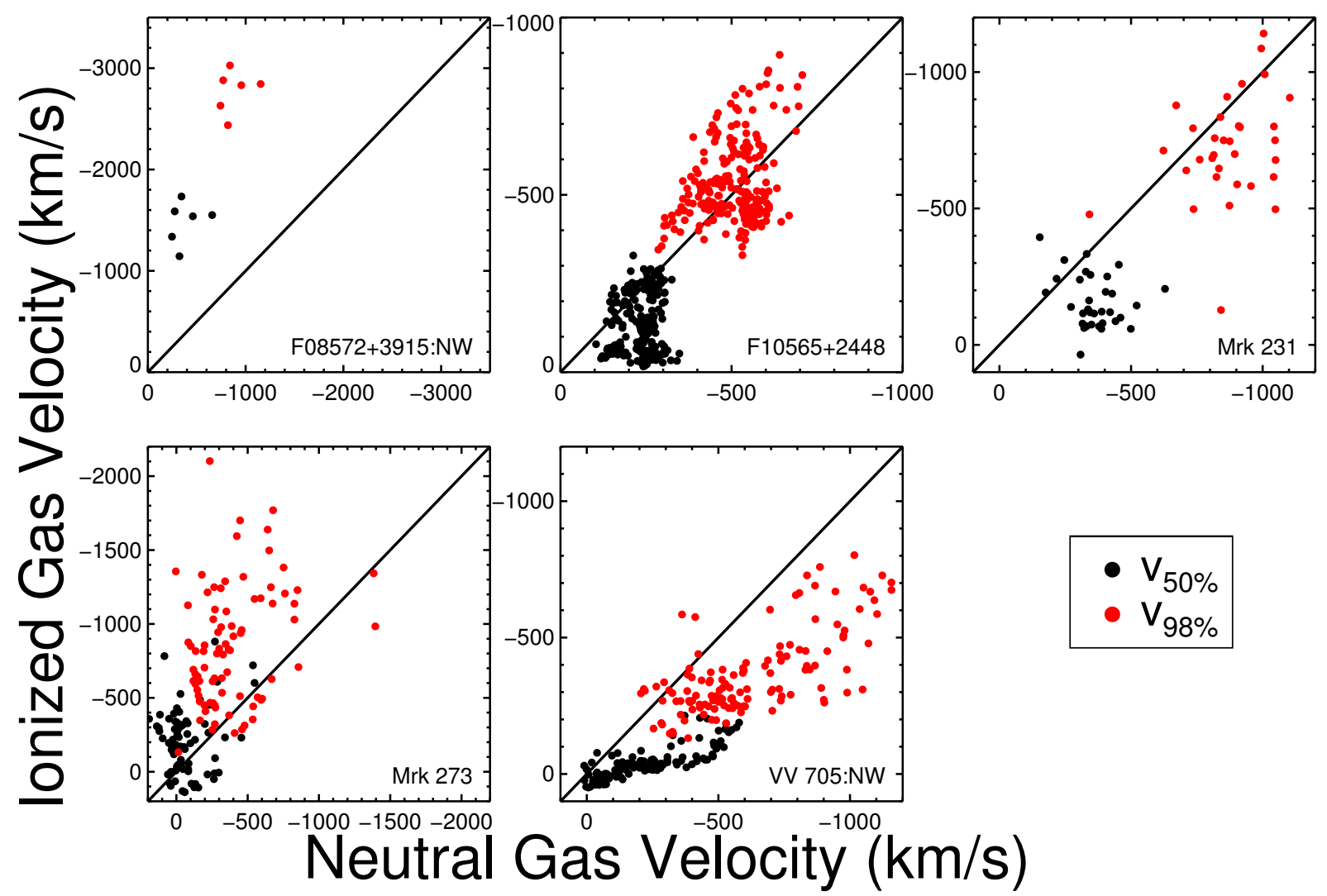

Figure 50. Ionized vs. neutral gas velocity, for the 5 nuclei in our sample with both ionized and neutral winds. Each point represents a spaxel with outflowing gas. The black points show $v_{50 \%}$, and the red points $v_{98 \%}$. The solid black line denotes equality. Within at least three systems (F10565+2448, Mrk 273, and VV 705:NW), there is a clear correlation between the velocities of the two gas phases $(\S 5.3$. It is unclear, however, what causes the deviations from equality, and why these deviations differ from object to object. 

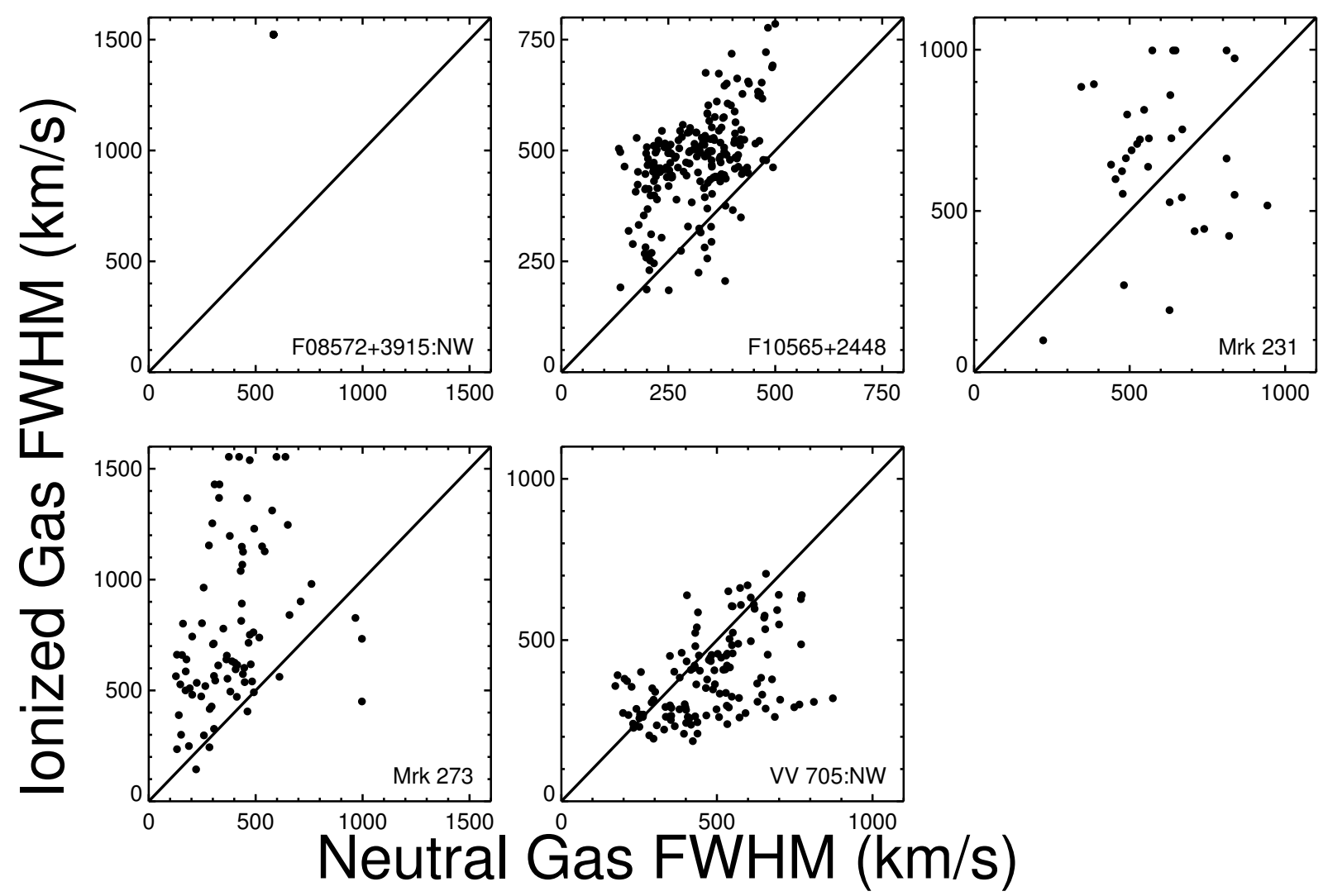

Figure 51. Ionized vs. neutral gas FWHM, for the 5 nuclei in our sample with both ionized and neutral winds. Each point represents a spaxel with outflowing gas. The solid black line denotes equality. Within at least three systems (F10565+2448, Mrk 273, and VV 705:NW), there is a clear correlation between the velocities of the two gas phases $(\S 5.3$. It is unclear, however, what causes the deviations from equality, and why these deviations differ from object to object. 


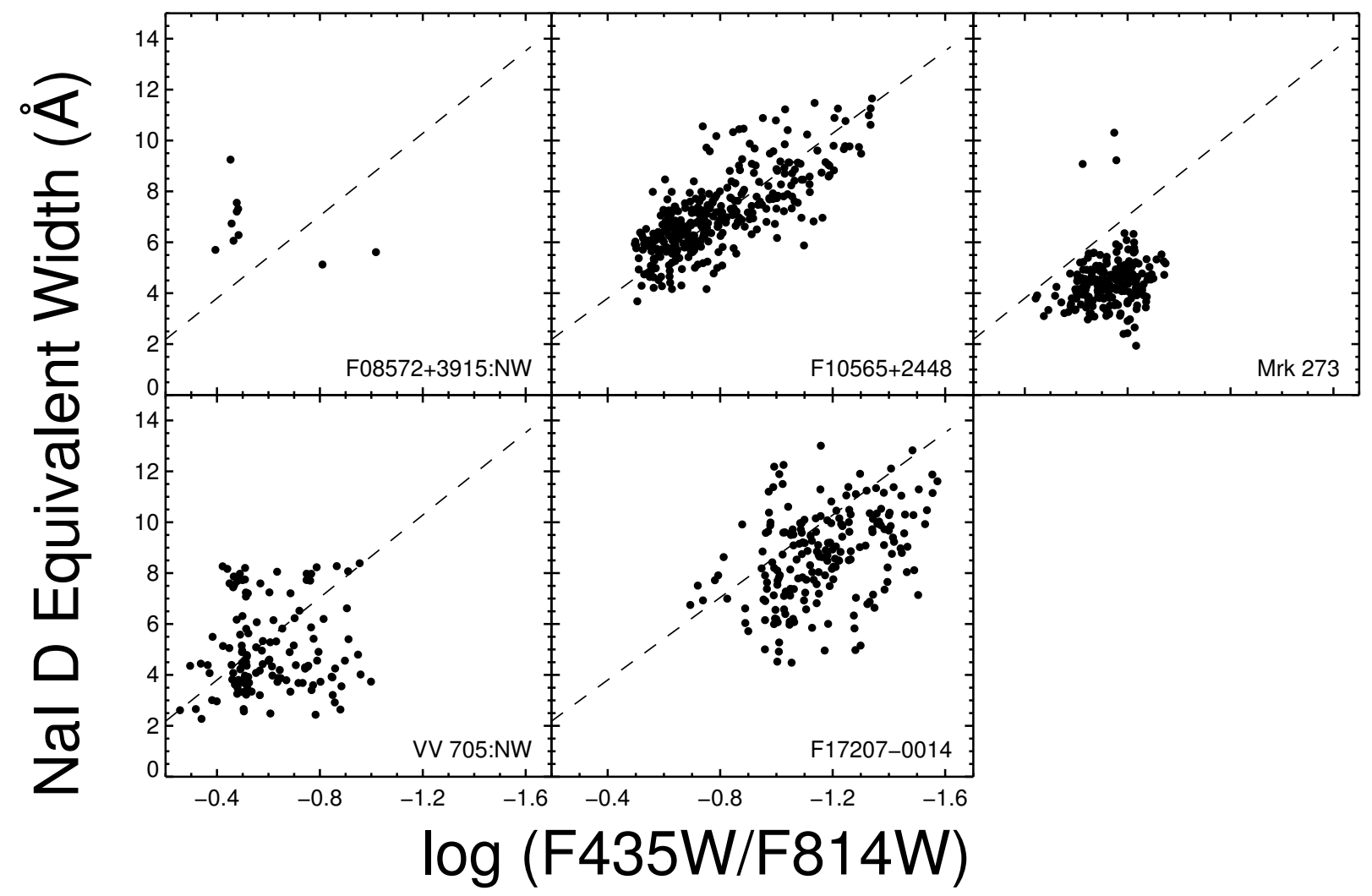

Figure 52. Rest-frame equivalent width of $\mathrm{Na}$ I D vs. $H S T$ color. The colors are binned and aligned to correspond to GMOS spaxels. The dashed line is a fit to F10565+2448. The correlations for F10565+2448 and F17207-0014 suggest that the dusty, neutral wind is attenuating the galaxy's continuum light. Though they have smaller dynamic range, the other systems fall near this correlation; these winds may also be dusty $(\S 5.3$. 


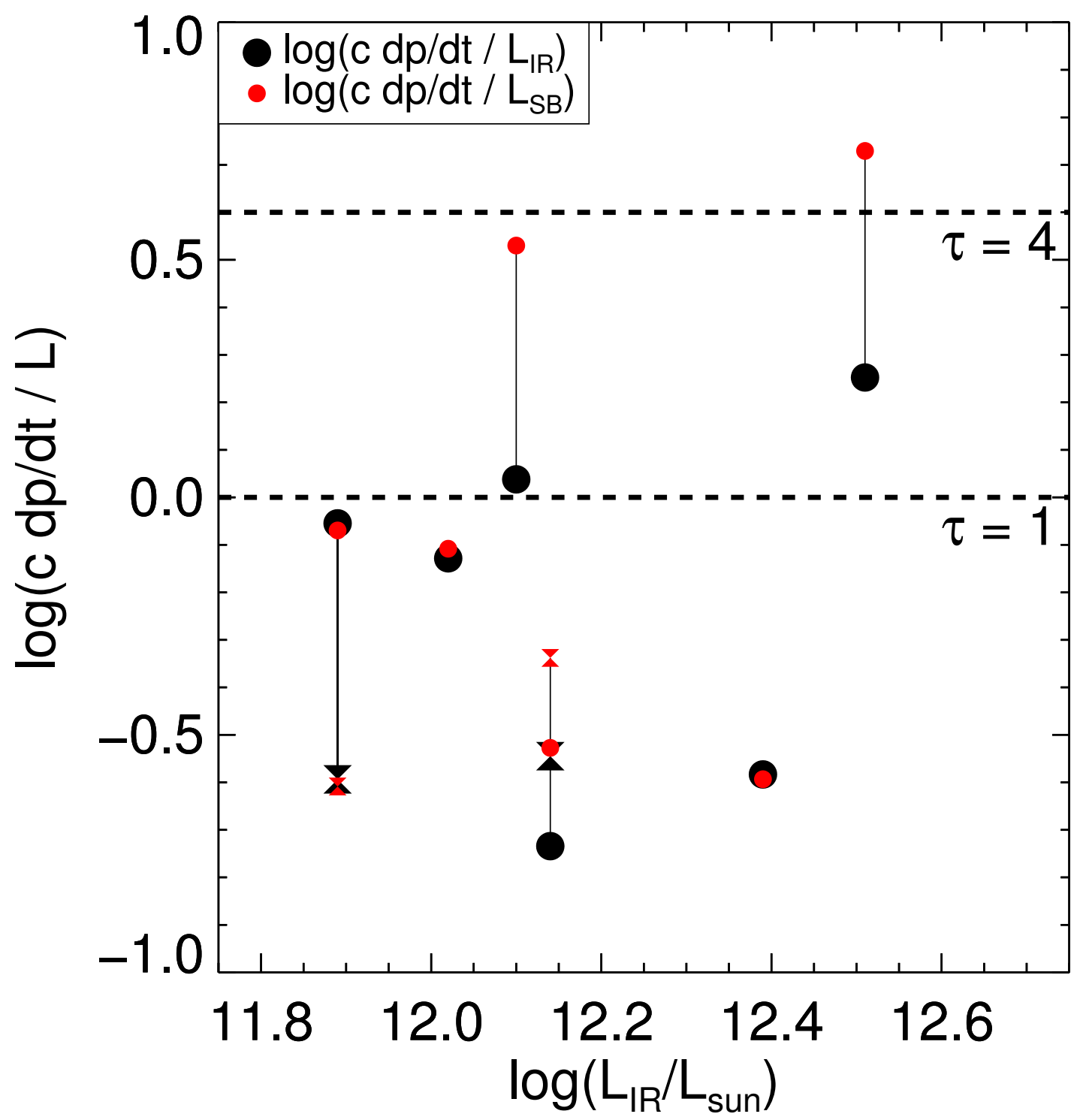

Figure 53. Momentum outflow rate normalized to infrared luminosity and starburst luminosity vs. infrared luminosity. Large, black symbols represent $c d p / d t / L_{I R}$, and small, red symbols represent $c d p / d t / L_{S B}$. Circles (hourglasses) are results from the SRFW (BSB) model. The dashed lines represent required optical depths of the outflow to photons if all of $L_{I R}$ or $L_{S B}$ is scattered by the wind. Note that powering the outflows with the starburst alone requires higher optical depths in some cases. 


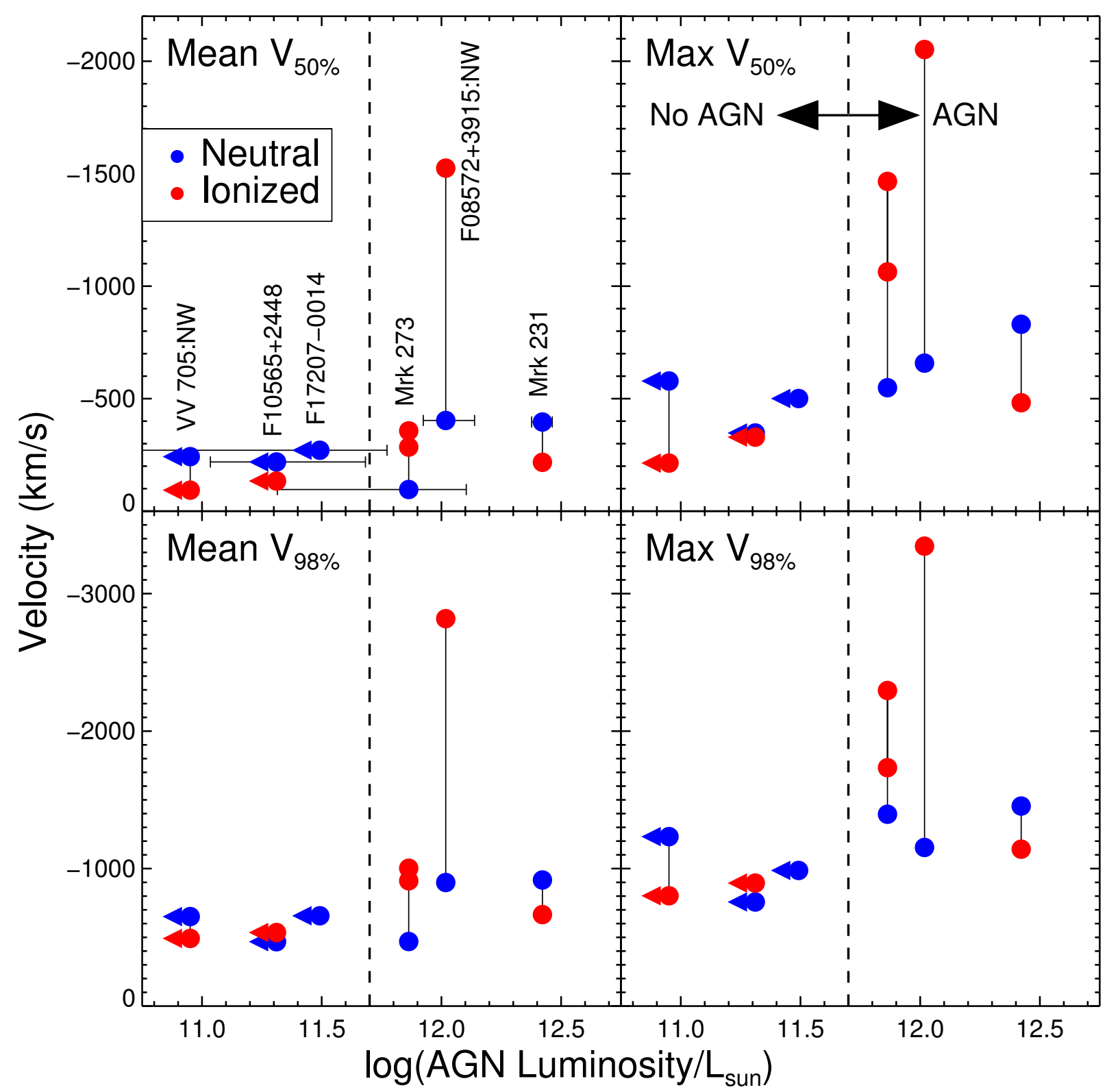

Figure 54. Velocity vs. AGN luminosity. Spatial averages and maximal values are computed from the projected velocities of outflowing components in all spaxels with outflowing gas. Blue (red) symbols represent neutral (ionized) gas. Two ionized gas symbols are shown for Mrk 273, representing the approaching and receding sides of the outflow. AGN luminosity is computed from the infrared luminosity and the average of six mid-infrared (MIR) diagnostics (Veilleux et al. 2009); the horizontal error bars represent the range of AGN luminosities determined from individual MIR diagnostics. The AGN luminosities for systems without clear multiwavelength evidence for an AGN are shown as upper limits. As labeled by the arrows in the upper right panel, the dashed line divides systems with and without clear evidence for an AGN. It is clear that galaxies with a luminous AGN (or QSO; F08572+3914:NW, Mrk 231, and Mrk 273) reach higher outflow velocities (up to $1450-3350 \mathrm{~km} \mathrm{~s}^{-1}$ in each object with a QSO; $\S 5.4 .3$. 


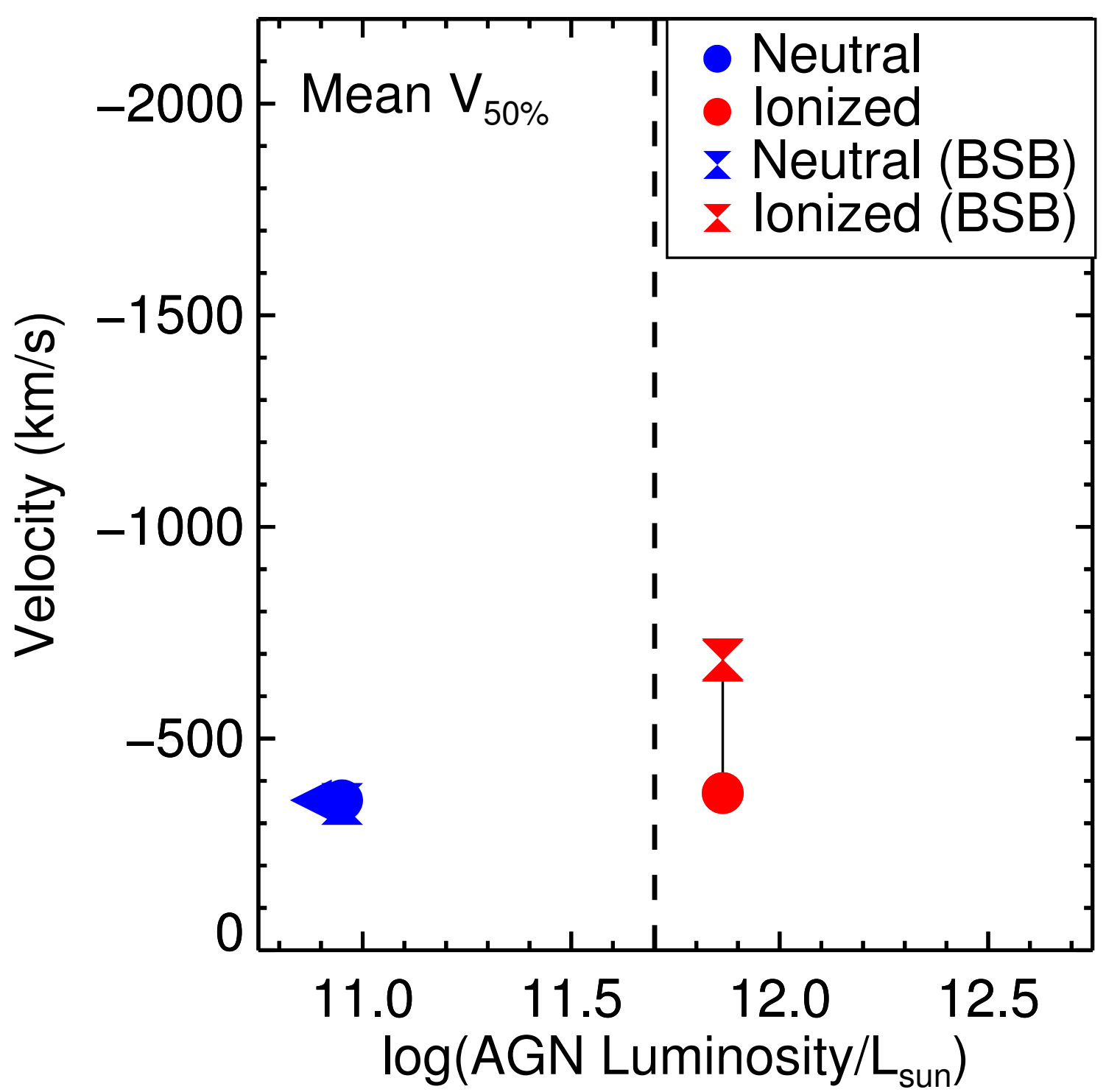

Figure 55. Velocity $\left(v_{50 \%}\right)$ vs. AGN luminosity for the two systems with a bipolar superbubble (Mrk 273 and VV 705:NW), to illustrate projection corrections to Figure 54 Hourglasses show average deprojected velocities from the BSB model, and circles show average projected velocities. To enter these averages, spaxels must fall within the superbubble boundary and contain significant absorption or emission. The average velocities in VV 705:NW change little on deprojection, since it is a face-on bubble, while those in the highly inclined Mrk 273 bubble roughly double $(\S 5.4 .3$. 


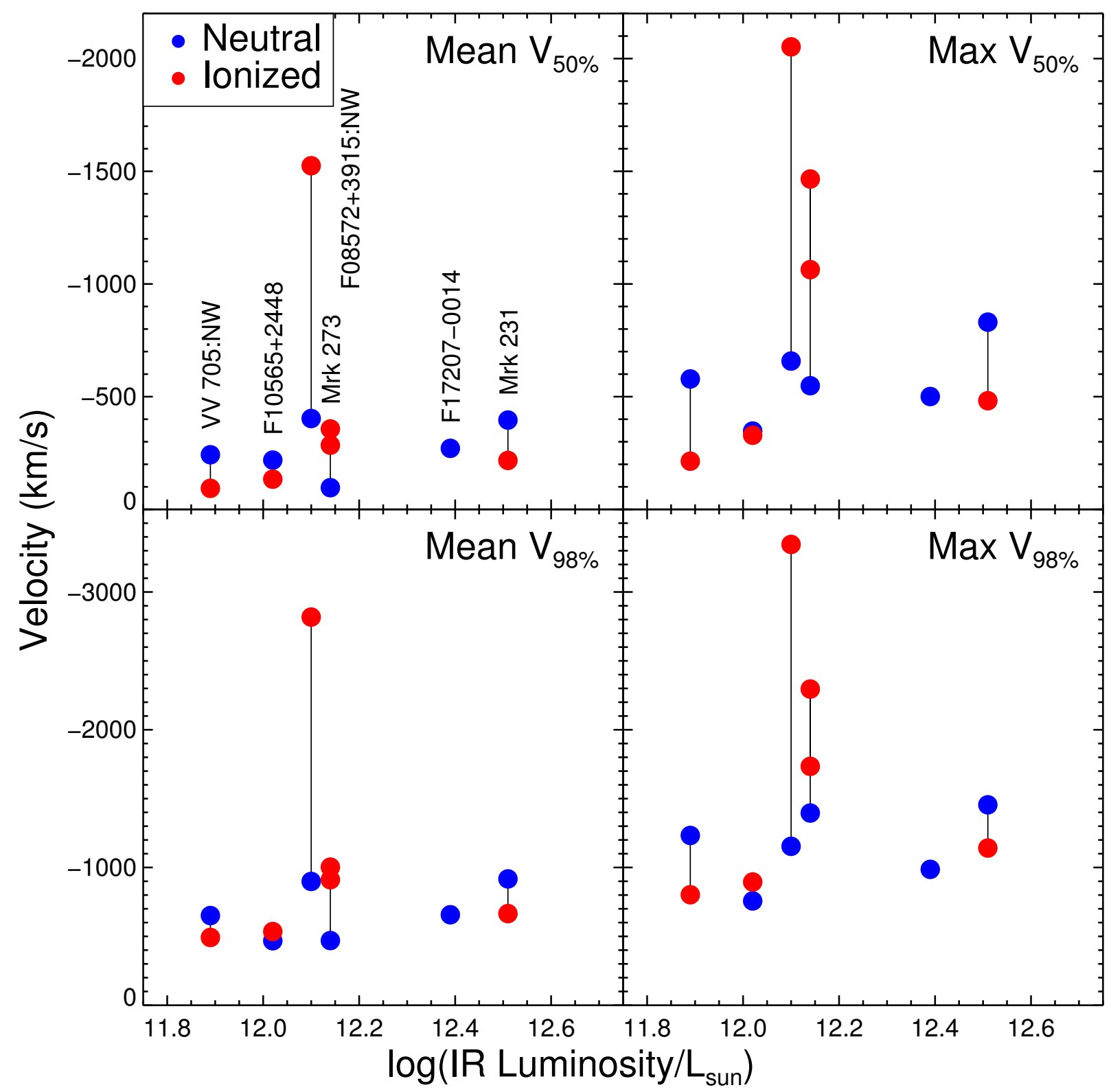

Figure 56. Velocity vs. total infrared luminosity (see Figure 54 for more details). There is no obvious relationship between velocity and infrared luminosity. The dynamic range in luminosity is only a factor of four, and the starburst and starburst+AGN systems are mixed; the sources with AGN are not the brightest. 
Table 1

Sample

\begin{tabular}{|c|c|c|c|c|c|c|c|c|c|}
\hline $\begin{array}{l}\text { Galaxy } \\
(1)\end{array}$ & $\begin{array}{c}\text { Other name } \\
(2)\end{array}$ & $\begin{array}{c}z \\
(3)\end{array}$ & $\begin{array}{c}\log \left(L_{\mathrm{IR}} / L_{\odot}\right) \\
(4)\end{array}$ & $\begin{array}{c}f_{25} / f_{60} \\
(5)\end{array}$ & $\begin{array}{c}\text { AGN Frac. } \\
(6)\end{array}$ & $\begin{array}{c}\text { SFR } \\
(7)\end{array}$ & $\begin{array}{c}\text { Spec. Type } \\
\text { (8) }\end{array}$ & $\begin{array}{c}\text { Merger Class } \\
\text { (9) }\end{array}$ & $\begin{array}{l}\text { Nuc. Sep. } \\
(10)\end{array}$ \\
\hline F08572+3915 & $\cdots$ & $0.0584(\mathrm{NW})$ & 12.10 & 0.241 & 0.72 & 61 & $\mathrm{H} / \mathrm{C}(\mathrm{NW})$ & IIIb & 5.0 \\
\hline & $\cdots$ & $0.0585(\mathrm{SE})$ & $\cdots$ & & & $\cdots$ & $\mathrm{H}(\mathrm{SE})$ & & $\cdots$ \\
\hline $\begin{array}{l}\text { F10565+2448 } \\
\text { Mrk 231 }\end{array}$ & $\cdots$ & 0.0431 & 12.02 & 0.105 & 0.17 & 150 & $\mathrm{C}$ & triple & triple \\
\hline & F12540+5708 & 0.0422 & 12.51 & 0.287 & 0.71 & 162 & S1 & IVb & 0.0 \\
\hline Mrk 273 & F13428+5608 & 0.0373 & 12.14 & 0.105 & 0.46 & 128 & S2 & IIIb & 1.0 \\
\hline VV 705 & $\begin{array}{c}\text { F15163+4255, } \\
\text { Mrk 848, I Zw } 107\end{array}$ & $0.04035(\mathrm{NW})$ & 11.89 & 0.157 & 0.10 & 120 & $\mathrm{C}(\mathrm{NW})$ & IIIIb & 7.5 \\
\hline .. & $\ldots$ & 0.0407 (SE) & . & & $\cdots$ & .. & C (SE) & $\ldots$ & $\ldots$ \\
\hline F17207-0014 & $\cdots$ & 0.0430 & 12.39 & 0.050 & 0.11 & 376 & $\mathrm{H}$ & IVa & 0.0 \\
\hline
\end{tabular}

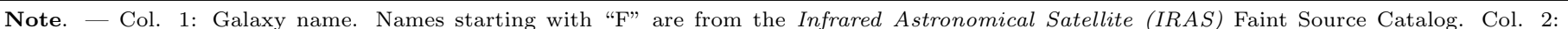

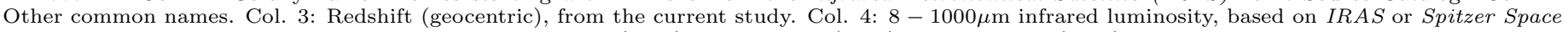

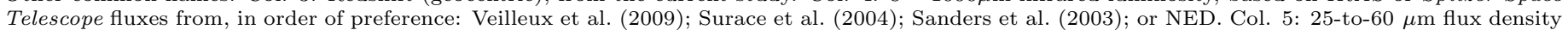

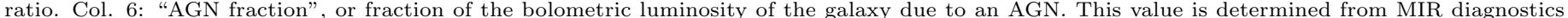

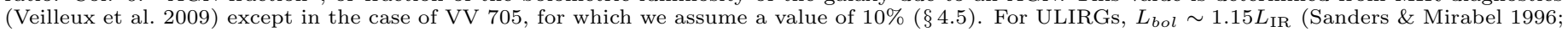

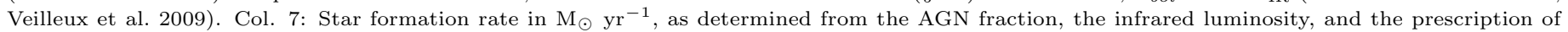

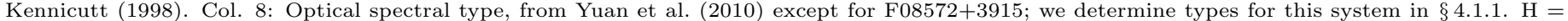

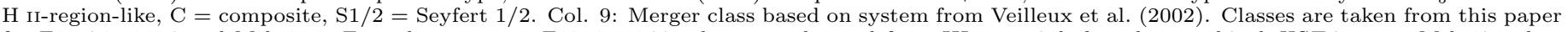

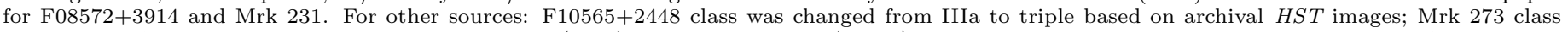

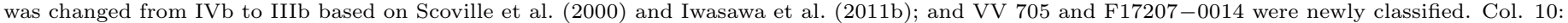

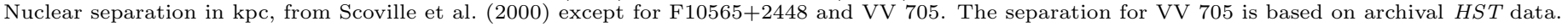

Table 2

Observations

\begin{tabular}{lcccc}
\hline \multicolumn{1}{c}{$\begin{array}{c}\text { Galaxy } \\
(1)\end{array}$} & $\begin{array}{c}\text { Dates } \\
(2)\end{array}$ & $\begin{array}{c}t_{\exp } \\
(3)\end{array}$ & $\begin{array}{c}\text { PA } \\
(4)\end{array}$ & $\begin{array}{c}\text { Dim } \\
(5)\end{array}$ \\
\hline F08572+3915:NW & 10apr14, 10may11, 10may19 & $3 \times 1800 \mathrm{~s}$ & $81^{\circ}$ & $16 \times 22$ \\
F08572+3915:SE & 10apr03, 10apr14, 10may19 & $4 \times 1800 \mathrm{~s}$ & $81^{\circ}$ & $16 \times 24$ \\
F10565+2448 & 07feb12 & $7 \times 1800 \mathrm{~s}$ & $90^{\circ}$ & $22 \times 26$ \\
Mrk 231 & 07jul19 & $5 \times 900 \mathrm{~s}$ & $35^{\circ}$ & $21 \times 25$ \\
Mrk 273 & 10jun13 & $4 \times 1800 \mathrm{~s}$ & $0^{\circ}$ & $20 \times 27$ \\
VV 705:NW & 10apr14, 10apr20, 10apr22 & $5 \times 1800 \mathrm{~s}$ & $35^{\circ}$ & $16 \times 22$ \\
VV 705:SE & 10apr14, 10apr20, 10apr22 & $4 \times 1800 \mathrm{~s}$ & $35^{\circ}$ & $16 \times 22$ \\
F17207-0014 & 10may09, 10may10, 10jun03 & $4 \times 1800 \mathrm{~s}$ & $90^{\circ}$ & $22 \times 30$ \\
\hline \multicolumn{5}{l}{ Note. - Col. 1: Galaxy name. Col. 2: UT dates of observations (in YYmmmDD } \\
format). Col. 3: Number of exposures $\times$ length of each exposure, in seconds. Col. \\
4: Position angle, E of N, of long axis of final data cube. Col. 5: Size of final data \\
cube, in units of 0.'3 spaxels.
\end{tabular}

Table 3

Outflow Velocity Statistics

\begin{tabular}{|c|c|c|c|c|c|c|c|}
\hline $\begin{array}{l}\text { Galaxy } \\
\text { (1) }\end{array}$ & $\begin{array}{l}\text { Phase } \\
(2)\end{array}$ & $\begin{array}{c}\langle\text { FWHM }\rangle \\
(3)\end{array}$ & $\begin{array}{c}\text { Max. FWHM } \\
(4)\end{array}$ & $\begin{array}{c}\left\langle v_{50 \%}\right\rangle \\
(5)\end{array}$ & $\begin{array}{c}\text { Max. } v_{50 \%} \\
(6)\end{array}$ & $\begin{array}{c}\left\langle v_{98 \%}\right\rangle \\
(7)\end{array}$ & $\begin{array}{c}\text { Max. } v_{98 \%} \\
(8)\end{array}$ \\
\hline \multirow[t]{2}{*}{ F08572+3915:NW } & neutral & 583 & 583 & -403 & -658 & -898 & -1153 \\
\hline & ionized & 1522 & 1522 & -1524 & -2052 & -2817 & -3345 \\
\hline \multirow[t]{2}{*}{ F10565+2448 } & neutral & 293 & 544 & -218 & -347 & -468 & -757 \\
\hline & ionized & 472 & 785 & -133 & -328 & -535 & -894 \\
\hline \multirow[t]{2}{*}{ Mrk 231} & neutral & 613 & 996 & -396 & -830 & -917 & -1455 \\
\hline & ionized & 527 & 997 & -217 & -482 & -665 & -1141 \\
\hline \multirow{2}{*}{$\begin{array}{l}\text { Mrk } 273 \\
\ldots\end{array}$} & neutral & 439 & 996 & -96 & -548 & -469 & -1395 \\
\hline & ionized (blue) & 760 & 1553 & -356 & -1465 & -1002 & -2295 \\
\hline \multirow{3}{*}{$\frac{\cdots}{\text { VV } 705: \mathrm{NW}}$} & ionized (red) & 737 & 1553 & 284 & 1063 & 911 & 1734 \\
\hline & neutral & 481 & 872 & -242 & -578 & -651 & -1232 \\
\hline & ionized & 469 & 705 & -93 & -213 & -492 & -802 \\
\hline F17207-0014 & neutral & 454 & 835 & -270 & -500 & -656 & -986 \\
\hline Starburst ULIRGs $^{\mathrm{a}}$ & neutral & 330 & & $\overline{-170}$ & & -450 & \\
\hline
\end{tabular}

Note. - Col. 2: Gas phase. Col. 3-4: Mean and maximum of full width at half maximum of velocity distribution, in $\mathrm{km} \mathrm{s}^{-1}$. Col. 5-6: Mean and maximum of velocity at center of (Gaussian) distribution. Col. 7-8: Mean and maximum of $v_{98 \%} \equiv v_{50 \%}-2 \sigma$.

a Computed from starburst-driven ULIRGs in Rupke et al. (2005c). This is a sample average, as opposed to the spatial averages in the current sample. 
Table 4

Single Radius Free Wind Parameters

\begin{tabular}{lccc}
\hline \hline Galaxy & Phase \\
$(1)$ & $(2)$ & $\begin{array}{c}\text { Radius } \\
(3)\end{array}$ & $\begin{array}{c}\Omega / 4 \pi \\
(4)\end{array}$ \\
\hline F08572+3915:NW & neutral & 2.0 & $0.06 / 0.02$ \\
$\ldots$ & ionized & 2.0 & 0.18 \\
\hline F10565+2448 & neutral & 5.0 & $0.17 / 0.12$ \\
$\ldots$ & ionized & 5.0 & 0.11 \\
\hline Mrk 231 & neutral & 3.0 & $0.24 / 0.14$ \\
$\cdots$ & ionized & 3.0 & 0.06 \\
\hline Mrk 273 & neutral & 4.0 & $0.05 / 0.03$ \\
$\cdots$ & ionized & 4.0 & 0.16 \\
\hline VV 705:NW & neutral & 3.0 & $0.14 / 0.08$ \\
$\cdots$ & ionized & 3.0 & 0.07 \\
\hline F17207-0014 & neutral & 5.0 & $0.10 / 0.06$ \\
\hline
\end{tabular}

Note. - Col. 2: Gas phase. Col. 3: Fixed wind radius, in kpc. Col. 4: Integrated covering factor, expressed as a fraction of $4 \pi$. The first number for the neutral phase does not include a correction for the fitted covering factor of the absorption line in each spaxel; the second does (see $\S 3.4 .1$.

Table 5

Bipolar Superbubble Parameters

\begin{tabular}{cccccccc}
\hline \hline $\begin{array}{c}\text { Galaxy } \\
(1)\end{array}$ & $\begin{array}{c}\text { Phase } \\
(2)\end{array}$ & $\begin{array}{c}R_{z} \\
(3)\end{array}$ & $\begin{array}{c}R_{x y} \\
(4)\end{array}$ & $\begin{array}{c}i \\
(5)\end{array}$ & $\begin{array}{c}\text { PA } \\
(6)\end{array}$ & $\begin{array}{c}v_{\max } \\
(7)\end{array}$ & $\begin{array}{c}n \\
(8)\end{array}$ \\
\hline Mrk 273 & ionized & $1.0 \pm 0.1$ & $0.8 \pm 0.1$ & $75 \pm 5^{\circ}$ & $-5 \pm 10^{\circ}$ & $1600 \pm 500$ & $2^{\mathrm{a}}$ \\
$\ldots$ & $\cdots$ & $1.0 \pm 0.1$ & $0.8 \pm 0.1$ & $75 \pm 5^{\circ}$ & $-5 \pm 10^{\circ}$ & $2100 \pm 500$ & $3^{\mathrm{a}}$ \\
VV 705:NW & neutral & $2 \pm 1$ & $1^{\mathrm{a}}$ & $6 \pm 6^{\circ}$ & $35 \pm 40^{\circ}$ & $500 \pm 150$ & $2^{\mathrm{a}}$ \\
$\cdots$ & $\cdots$ & $3 \pm 2$ & $1^{\mathrm{a}}$ & $4 \pm 4^{\circ}$ & $35 \pm 40^{\circ}$ & $600 \pm 150$ & $3^{\mathrm{a}}$
\end{tabular}

Note. - Col. 2: Gas phase. Col. 3-4: Semi-principal axes of the ellipsoid bubble, in kpc. The $z$ axis is the common axis shared by the two counterpropagating bubbles in each system. Col. 5: The inclination of the bubble's $z$ axis with respect to the line of sight (for $i=0^{\circ}$, the $z$ axis and LOS are parallel). Col. 6: The position angle of the bubble's $z$ axis (for PA $=0^{\circ}$, the $z$ axis of the near side bubble is directed $\mathrm{N}$ ). Col. 7: The (maximum) velocity of the bubble along the $z$ axis, in $\mathrm{km} \mathrm{s}^{-1}$. Col. 8: The power-law index of the bubble's velocity field: $v=v_{z}\left(r / r_{z}\right)^{n}$, where $r$ is the distance from the center of the wind, and $v_{z}$ and $r_{z}=2 R_{z}$ are the velocity and distance along the $z$ axis.

a This parameter is fixed. 
Table 6

Mass, Momentum, and Energy

\begin{tabular}{|c|c|c|c|c|c|c|c|c|}
\hline $\begin{array}{c}\text { Galaxy } \\
(1)\end{array}$ & $\begin{array}{c}\text { phase } \\
(2)\end{array}$ & $\begin{array}{c}\text { model } \\
(3)\end{array}$ & $\begin{array}{c}M \\
M_{\odot} \\
(4)\end{array}$ & $\begin{array}{l}d M / d t \\
M_{\odot} / \mathrm{yr} \\
\quad(5)\end{array}$ & $\begin{array}{c}p \\
\text { dyne s } \\
(6)\end{array}$ & $\begin{array}{c}c \times d p / d t \\
L_{\odot} \\
(7)\end{array}$ & $\begin{array}{c}E \\
\text { erg } \\
(8)\end{array}$ & $\begin{array}{c}d E / d t \\
\mathrm{erg} / \mathrm{s} \\
(9)\end{array}$ \\
\hline $\mathrm{F} 08572+3915: \mathrm{NW}$ & neutral & SRFW & 7.88 & 1.39 & 48.98 & 11.95 & 56.69 & 42.78 \\
\hline$\ldots$ & ionized & SRFW & 6.93 & 0.88 & 48.47 & 11.84 & 56.58 & 43.05 \\
\hline$\ldots$ & total & SRFW & 7.92 & 1.51 & 49.10 & 12.20 & 56.94 & 43.24 \\
\hline F10565+2448 & neutral & SRFW & 9.10 & 1.81 & 49.80 & 11.95 & 57.17 & 42.42 \\
\hline ... & ionized & SRFW & 7.88 & 0.16 & 48.15 & 10.00 & 56.06 & 40.93 \\
\hline$\cdots$ & total & SRFW & 9.12 & 1.82 & 49.81 & 11.95 & 57.20 & 42.44 \\
\hline Mrk 231 & neutral & SRFW & 8.92 & 2.25 & 50.02 & 12.82 & 57.74 & 43.68 \\
\hline$\ldots$ & ionized & SRFW & 6.97 & -0.10 & 47.67 & 10.29 & 55.50 & 41.17 \\
\hline$\ldots$ & total & SRFW & 8.92 & 2.25 & 50.02 & 12.82 & 57.74 & 43.69 \\
\hline Mrk 273 & neutral & SRFW & 8.23 & 0.90 & 48.79 & 11.07 & 56.50 & 41.87 \\
\hline$\ldots$ & ionized & SRFW & 8.07 & 0.87 & 48.77 & 11.25 & 56.76 & 42.27 \\
\hline$\ldots$ & total & SRFW & 8.46 & 1.19 & 49.08 & 11.47 & 56.95 & 42.41 \\
\hline$\ldots$ & ionized & BSB & 7.27 & 1.18 & 48.35 & 11.66 & 56.36 & 42.77 \\
\hline VV 705:NW & neutral & SRFW & 8.53 & 1.59 & 49.36 & 11.89 & 56.97 & 42.61 \\
\hline$\ldots$ & ionized & SRFW & 7.66 & 0.28 & 48.05 & 10.13 & 55.92 & 41.09 \\
\hline$\ldots$ & total & SRFW & 8.59 & 1.61 & 49.38 & 11.90 & 57.01 & 42.62 \\
\hline$\ldots$ & neutral & BSB & 8.22 & 1.33 & 48.86 & 11.36 & 56.55 & 42.16 \\
\hline F17207-0014 & neutral & SRFW & 8.63 & 1.53 & 49.52 & 11.87 & 57.05 & 42.52 \\
\hline
\end{tabular}

Note. - Col. (2): Gas phase. Col. (3): Outflow model. SRFW = Single radius, mass conserving free wind. BSB = Superbubble. Col. (4)-(9): Logarithms of the mass, mass outflow rate, momentum, momentum outflow rate, energy, and energy outflow rate contained in the wind ( $\S 3.4$. The ionized gas values depend on electron density as $n_{e}^{-1}$, and we assume $n_{e}=10 \mathrm{~cm}^{-3}$. The neutral gas values depends on ionization fraction $y$ as $(1-y)^{-1}$, and we assume $y=0.9$. Note that correcting the ionized gas for reddening will increase the values in this phase, as well as the total.

Table 7

Percent of Mass, Momentum, and Energy in Neutral Phase

\begin{tabular}{lccccccc}
\hline \hline \multicolumn{1}{c}{$\begin{array}{c}\text { Galaxy } \\
(1)\end{array}$} & $\begin{array}{c}\text { model } \\
(2)\end{array}$ & $\begin{array}{c}M \\
(3)\end{array}$ & $\begin{array}{c}d M / d t \\
(4)\end{array}$ & $\begin{array}{c}p \\
(5)\end{array}$ & $\begin{array}{c}d p / d t \\
(6)\end{array}$ & $\begin{array}{c}E \\
(7)\end{array}$ & $\begin{array}{c}d E / d t \\
(8)\end{array}$ \\
\hline F08572+3915:NW & SRFW & 89 & 76 & 76 & 56 & 56 & 34 \\
F10565+2448 & SRFW & 94 & 97 & 97 & 98 & 92 & 96 \\
Mrk 231 & SRFW & 98 & 99 & 99 & 99 & 99 & 99 \\
Mrk 273 & SRFW & 59 & 51 & 51 & 39 & 35 & 28 \\
VV 705:NW & SRFW & 88 & 95 & 95 & 98 & 91 & 97 \\
\hline
\end{tabular}

Note. - Col. 2: Outflow model. SRFW $=$ Single radius, mass conserving free wind. BSB $=$ Superbubble. Col. $3-8$ : Percent of the mass, mass outflow rate, momentum, momentum outflow rate, energy, and energy outflow rate in the neutral phase of the wind (compared to the total in the neutral and ionized phases). Note that correcting the ionized gas for reddening will decrease these values. 
Table 8

Power Sources and Feedback

\begin{tabular}{|c|c|c|c|c|c|c|c|}
\hline $\begin{array}{c}\text { Galaxy } \\
(1)\end{array}$ & $\begin{array}{c}\text { phase } \\
(2)\end{array}$ & $\begin{array}{c}\text { model } \\
(3)\end{array}$ & $\begin{array}{c}c \times d p / d t / \\
L_{\mathrm{IR}} \\
(4)\end{array}$ & $\begin{array}{c}d E / d t / \\
d E / d t_{S B} \\
(5)\end{array}$ & $\begin{array}{c}d E / d t / \\
L_{A G N} \\
(6)\end{array}$ & $\begin{array}{c}d M / d t / \\
S F R \\
(7)\end{array}$ & $\begin{array}{c}d M / d t / \\
d M / d t_{S B} \\
\text { (8) }\end{array}$ \\
\hline F08572+3915:NW & neutral & SRFW & -0.21 & -0.45 & $>-2.88$ & -0.39 & 0.58 \\
\hline & ionized & SRFW & -0.32 & -0.17 & $>-2.61$ & -0.90 & 0.08 \\
\hline 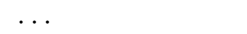 & total & SRFW & 0.04 & 0.01 & $>-2.42$ & -0.28 & 0.70 \\
\hline F10565+2448 & neutral & SRFW & -0.13 & -1.19 & -2.53 & -0.36 & 0.62 \\
\hline$\ldots$ & ionized & SRFW & -2.08 & -2.68 & -4.02 & -2.01 & -1.03 \\
\hline$\ldots$ & total & SRFW & -0.13 & -1.18 & -2.52 & -0.35 & 0.63 \\
\hline Mrk 231 & neutral & SRFW & 0.25 & 0.03 & $>-2.38$ & 0.04 & 1.02 \\
\hline$\ldots$ & ionized & SRFW & -2.28 & -2.48 & $>-4.90$ & -2.31 & -1.33 \\
\hline$\ldots$ & total & SRFW & 0.25 & 0.04 & $>-2.38$ & 0.05 & 1.03 \\
\hline Mrk 273 & neutral & SRFW & -1.13 & -1.68 & $>-3.64$ & -1.21 & -0.23 \\
\hline$\ldots$ & ionized & SRFW & -0.95 & -1.28 & $>-3.24$ & -1.23 & -0.25 \\
\hline$\ldots$ & total & SRFW & -0.73 & -1.14 & $>-3.09$ & -0.92 & 0.06 \\
\hline$\cdots$ & ionized & BSB & -0.54 & -0.78 & $>-2.74$ & -0.93 & 0.05 \\
\hline VV 705:NW & neutral & SRFW & -0.06 & -0.91 & -1.99 & -0.49 & 0.49 \\
\hline & ionized & SRFW & -1.82 & -2.44 & -3.51 & -1.80 & -0.82 \\
\hline$\ldots$ & total & SRFW & -0.05 & -0.90 & -1.97 & -0.47 & 0.51 \\
\hline$\ldots$ & neutral & BSB & -0.59 & -1.36 & -2.44 & -0.75 & 0.23 \\
\hline F17207-0014 & neutral & SRFW & -0.58 & -1.49 & -2.61 & -1.05 & -0.07 \\
\hline
\end{tabular}

Note. - Col. 2: Gas phase. Col. 3: Outflow model. SRFW = Singe radius, mass conserving free wind. $\mathrm{BSB}=$ Superbubble. Col. 4: Logarithm of the momentum outflow rate divided by the momentum input rate if all of the galaxy's infrared luminosity accelerates the wind and each photon is intercepted once. Col. 5: Logarithm of the energy outflow rate divided by the mechanical energy production rate from a continuous starburst (Leitherer et al. 1999). Col. 6: Logarithm of the energy outflow rate divided by the AGN luminosity. Col. 7: Logarithm of mass outflow rate normalized to the star formation rate. Col. 8: Logarithm of the mass outflow rate divided by the hot gas mass production rate expected from a continuous starburst (Leitherer et al. 1999). 